Mathematics and Computer

$\frac{52}{7-2-97} 950$

Science Division ANL-97/11

Mathematics and Computer

Science Division

Mathematics and Computer

Science Division

Mathematics and Computer

Science Division

Mathematics and Computer

Science Division

Mathematics and Computer

Science Division

Mathematics and Computer

Science Division

Mathematios and Computer

Science Division

Mathemalics and Computer

Sclence Division

Mathematics and Computer

Sclence Division

Wrathematics and Computer

Science Division

Mathemalics and Computer

Science Division

Mathematics and Comouter

by J. M. Herbert

Science Division

Mathematics and Computer

Science Division

Mathematics and Computer

Science Division

Wathematics and Computer

Science Division

Mathematics and Computer

Science Division

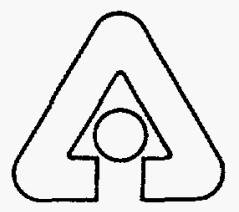

Argonne National Laboratory, Argonne, Illinois 60439

operated by The University of Chicago

for the United States Department of Energy under Contract W-31-109-Eng-38

Mathematics and Computer

Sclence Division

Mathematics and Computer

Sclence Division

Wathematics and Computer

Sclence Division

Mathematics and Computer

Science Division 
Argonne National Laboratory, with facilities in the states of Illinois and Idaho, is owned by the United States government, and operated by The University of Chicago under the provisions of a contract with the Department of Energy.

\section{DISCLAIMER}

This report was prepared as an account of work sponsored by an agency of the United States Government. Neither the United States Government nor any agency thereof, nor any of their employees, makes any warranty, express or implied, or assumes any legal liability or responsibility for the accuracy, completeness, or usefulness of any information, apparatus, product, or process disclosed, or represents that its use would not infringe privately owned rights. Reference herein to any specific commercial product, process, or service by trade name, trademark, manufacturer, or otherwise, does not necessarily constitute or imply its endorsement, recommendation, or favoring by the United States Government or any agency thereof. The views and opinions of authors expressed herein do not necessarily state or reflect those of the United States Government or any agency thereof.

Reproduced from the best available copy.

Available to DOE and DOE contractors from the

Office of Scientific and Technical Information

$$
\text { P.O. Box } 62
$$

Oak Ridge, TN 37831

Prices available from (423) 576-8401

Available to the public from the

National Technical Information Service

U.S. Department of Commerce

5285 Port Royal Road

Springtield, VA 22161 
Symbolic Derivation of High-Order Rayleigh-Schrödinger Perturbation Energies Using Computer Algebra: Application to Vibrational-Rotational Analysis of Diatomic Molecules

by

John M. Herbert

SUBMITTED IN PARTIAL FULFILLMENT OF THE RESEARCH REQUIREMENTS FOR THE BSc DEGREE

\author{
DEPARTMENT OF CHEMISTRY \\ KANSAS STATE UNIVERSITY \\ MANHATTAN, KANSAS
}

Signed irtalte C. Simler

Walter C. Ermler

Project Supervisor 


\section{DISCLAIMIER}

Portions of this document may be illegible in electronic image products. Images are produced from the best available original document. 


\section{CONTENTS}

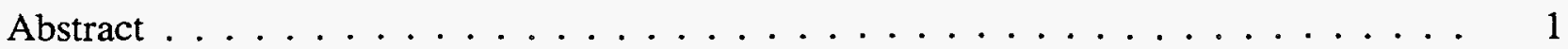

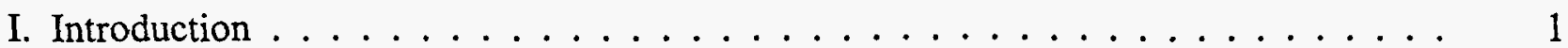

II. The Nuclear Schrödinger Equation . . . . . . . . . . . . . . . . . . 4

III. The Harmonic Oscillator and Rigid-Rotator Models . . . . . . . . . . . . . . . 5

IV. Anharmonicity, Coupling, and Centrifugal Distortion . . . . . . . . . . . 8

V. Perturbation Theory for a Diatomic Molecule . . . . . . . . . . . . . 10

VI. Evaluation of Matrix Elements . . . . . . . . . . . . . . . . . 12

VII. Rayleigh-Schrödinger Expansions . . . . . . . . . . . . . . . . . . . 16

VIII. Energy Corrections . . . . . . . . . . . . . . . . . . . . 19

XX. Implementation Using Mathematica . . . . . . . . . . . . . . . . . . . 22

X. Numerical Results and Analysis . . . . . . . . . . . . . . . . 26

XI. Conclusion . . . . . . . . . . . . . . . . . . . . 39

Appendices

A. The Mathematica Package RSPERTURB . . . . . . . . . . . . . . . . . . 42

B. The Mathematica Package DiatomicVIBRot . . . . . . . . . . . . . 46

C. Second-Order Correction Formulae . . . . . . . . . . . . . . 47

D. Fourth-Order Correction Formulae . . . . . . . . . . . . . . . 49

E. Sixth-Order Correction Formulae . . . . . . . . . . . . . . . 52

F. Hulburt-Hirschfelder Force Constants . . . . . . . . . . . . . . 59

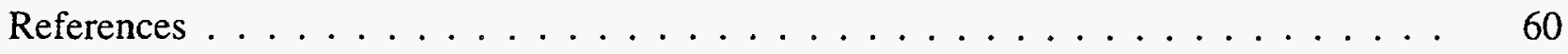




\title{
Symbolic Derivation of High-Order Rayleigh-Schrödinger Perturbation Energies Using Computer Algebra: Application to Vibrational-Rotational Analysis of Diatomic Molecules
}

by

John M. Herbert

\begin{abstract}
Rayleigh-Schrödinger perturbation theory is an effective and popular tool for describing low-lying vibrational and rotational states of molecules. This method, in conjunction with ab initio techniques for computation of electronic potential energy surfaces, can be used to calculate first-principles molecular vibrational-rotational energies to successive orders of approximation. Because of mathematical complexities, however, such perturbation calculations are rarely extended beyond the second order of approximation, although recent work by Herbert has provided a formula for the $n$ th-order energy correction. This report extends that work and furnishes the remaining theoretical details (including a general formula for the RayleighSchrödinger expansion coefficients) necessary for calculation of energy corrections to arbitrary order. The commercial computer algebra software Mathematica is employed to perform the prohibitively tedious symbolic manipulations necessary for derivation of generalized energy formulae in terms of universal constants, molecular constants, and quantum numbers. As a pedagogical example, a Hamiltonian operator tailored specifically to diatomic molecules is derived, and the perturbation formulae obtained from this Hamiltonian are evaluated for a number of such molecules. This work provides a foundation for future analyses of polyatomic molecules, since it demonstrates that arbitrary-order perturbation theory can successfully be applied with the aid of commercially available computer algebra software.
\end{abstract}

\section{INTRODUCTION}

Molecular vibrational-rotational (or vibro-rotational) energy levels are obtained from theory by solving (in some approximate fashion) the quantum-mechanical Schrödinger equation representing the internal nuclear motion of the molecule. These energies-which are eigenvalues of the quantum-mechanical Hamiltonian operator associated with internal nuclear motion-and their corresponding eigenfunctions are of key interest because numerous molecular properties such as equilibrium geometric structure, bond lengths and polarities, dissociation energies and other thermodynamic quantities, moments of inertia, and stability of transition states are linked to internal nuclear motion $[1,2]$. While most of these properties are obtainable via experimental spectroscopy, theoretical calculation and analysis of vibrational-rotational spectra are vital to the 
study of molecules not readily amenable to experimental investigation; these include unstable species, weakly bound complexes, and highly toxic compounds [2].

To approximate solutions to the nuclear Schrödinger equation, most researchers enlist either variational methods or perturbation theory [3]. While variational procedures are somewhat more accurate than perturbation methods, the difference is small for low-lying energy levels and is inevitably overshadowed by error introduced during calculation of $a b$ initio potential energy surfaces [4]. Typically, perturbation theory provides vibrational and rotational data that are as accurate as any currently available $a b$ initio potential energy surface will allow [5]. In addition, perturbation theory holds an advantage over variation in that the form of the wave functions is necessarily shaped by the nature of the perturbation [6], whereas with variation the choice of trial function is essentially arbitrary [7]. As a result, perturbation theory remains the method of choice for describing the low-lying vibrational and rotational states of polyatomic molecules [8]'.

Perturbation procedures furnish successively higher-order correction terms to eigenvalues and eigenfunctions; with suitable convergence this method can, in principle, be extended to arbitrary order, until the correction terms become negligibly small. In practice, however, the calculation of high-order corrections to vibrational-rotational energies and wave functions is limited by the accuracy of the potential energy surface and by the inherent complexity of the perturbation formulae themselves [9].

Fortunately, advances in high-speed computing over the past two decades have somewhat assuaged the former problem and have made feasible $[4,10]$ the calculation of accurate ab initio potential energy surfaces for small molecules [11-14]. Once nuclear and electronic motions are separated via the Born-Oppenheimer approximation [15], the electronic Schrödinger equation is solved for a number of nuclear configurations; this data is then fit to an analytic function-a potential energy surface-that provides electronic energy as a function of nuclear configuration [16]. This energy function is subsequently used as the potential energy operator in the Schrödinger equation for nuclear motion [3].

The second problem with high-order perturbation theory, and one that has yet to be satisfactorily resolved, is that even relatively low-order perturbation calculations involve prohibitively massive algebraic expressions. Because of this complexity, analytic perturbation theory is seldom applied beyond second order [3,17], and instead variation-perturbation methods

\footnotetext{
${ }^{1}$ For highly excited states, the convergence behavior of perturbation procedures is poor, and variational methods are significantly more accurate [4].
} 
[18] or numerical solution of the perturbed Schrödinger equation [8] is employed to calculate high-order correction terms. However, whereas derivation of an analytic formula for each perturbation correction requires no a priori knowledge of molecular data and therefore provides a general expression that can be applied to any system (upon substitution of the appropriate molecular constants), numerical techniques require that molecular parameters be inserted into equations before these equations are solved. Thus, the numerical procedure must be repeated for each change in parameters.

Although manual computation of explicit algebraic correction formulae to arbitrary order is not feasible, the growing availability of algebraic software capable of large-scale symbolic manipulations offers the possibility of obtaining the desired expressions via computer. To this end, high-order perturbation theory has been successfully applied to some simple systems using computer algebra [19-23]; these applications, however, are limited in scope to a single system [20, $22,23]$ or a small group of similar systems [19,21], and in all cases the systems are composed of atoms and not molecules. Furthermore, these authors take advantage of the Hellmann-Feynman and Hypervirial theorems [3,6] in order to circumvent explicit computation of wave functions. Calculation of a vibrational-rotational wave function, however, allows one to compute expectation values and molecular properties other than energy [2].

First attempts at a more general computer algebra-based approach to a perturbation problem were presented in a series of papers by Bouanich ${ }^{2}$ in which the author uses commercial algebra software to derive symbolic algebraic formulae for integrals arising in a perturbation treatment of the vibration and rotation of diatomic molecules. Due to the nature of the potential energy function employed, however, Bouanich [25] is unable to extend these results to arbitrary order of correction. More recently, Dudas et al. [9] have developed a computer program (suitable for implementation in the commercial algebraic software package Mathematica) that can derive vibrational-rotational integrals to arbitrary order of correction, although these authors provide no details concerning application of their algorithm to a vibrational-rotational analysis problem.

This report presents a perturbation-theoretical analysis of the vibration and rotation of diatomic molecules. Using a modified form of the general perturbation energy formula developed by Herbert [26] and incorporating the Mathematica code described above, explicit algebraic formulae for energy and wave function correction terms are derived in the Mathematica environment. These expressions incorporate universal and molecular constants strictly in

\footnotetext{
${ }^{2}$ For a summary with appropriate citations, see [24] or [25].
} 
symbolic form, so that the solution is not specific to a single $m^{\prime}$ cule. Thus, after an initial time investment to derive these formulae, it is a simple matter to sub itute appropriate constants and thereby calculate vibro-rotational energies to arbitrary order of correction for any diatomic molecule to which the theory applies. Furthermore, use of the Rayleigh-Schrödinger form of perturbation theory facilitates explicit calculation of vibrational-rotational wave functions, and from these equations of state numerous molecular properties may be calculated [2].

Although perturbation theory is neither the fastest nor the most accurate procedure for vibrational-rotational analysis of diatomic molecules [8], this work is nonetheless significant because for the first time perturbation corrections can be calculated accurately, efficiently, and systematically for any order of correction. These results stand primarily as a pedagogical precursor meant to furnish important insight and provide a framework for future studies of the vibration and rotation of polyatomic molecules, where perturbation theory is the most common method of analysis [8].

\section{The Nuclear Schrödinger EQuation}

In considering vibrational and rotational energies, one is concerned only with motion internal to a molecule, so translational motion is discounted. When written exclusively for the internal nuclear motion of a diatomic molecule, the time-independent Schrödinger equation governing rotation and vibration is

$$
\left[-\frac{\hbar^{2}}{2 \mu} \hat{\nabla}^{2}+\mathrm{U}(R)\right] \psi_{N}=E \psi_{N},
$$

where $E$ is the system's internal energy (i.e., the total energy less translational and electronic contributions), $\mathrm{U}(R)$ is the molecular potential energy function, and $\psi_{N}$ is the stationary-state wave function for nuclear motion in a reference frame that translates with the molecule. The constants $\mu$ and $\hbar$ in (1) are, respectively, the system's reduced mass and Dirac's constant (which is equal to Planck's constant divided by $2 \pi$ ). The first bracketed term in (1) is the kinetic energy operator for a diatomic molecule; its explicit form will not be required.

For a diatomic molecule, the potential energy $U$ depends exclusively on the internuclear separation $R$. Thus, the diatomic potential is spherically symmetric [17], so the eigenfunctions in (1) have the form

$$
\psi_{N}=\mathrm{F}(R) \mathrm{Y}_{J}^{M}(\theta, \phi)
$$


where $\mathrm{Y}_{J}^{M}(\theta, \phi)$ is the well-known spherical harmonic function [3] and $J$ and $M$ are angular momentum quantum numbers. The unknown radial factor $\mathrm{F}(R)$ is some function that depends solely on the internuclear separation; this function can be shown [3] to obey the so-called radial differential equation:

$$
-\frac{\hbar^{2}}{2 \mu}\left(\mathrm{F}^{\prime \prime}(R)+\frac{2}{R} \mathrm{~F}^{\prime}(R)\right)+\frac{J(J+1) \hbar^{2}}{2 \mu R^{2}} \mathrm{~F}(R)+\mathrm{U}(R) \mathrm{F}(R)=E \mathrm{~F}(R)
$$

\section{THE HARMONIC OSCILlATOR AND RIGID-Rotator MODELS}

To fully specify the solutions (2) to the nuclear Schrödinger equation (1), one must solve (3) to obtain an expression for the radial factor $\mathrm{F}(R)$. While this procedure is well established and can be found in many quantum chemistry textbooks, some elaboration concerning solution of (3) is necessary to elucidate how vibrational and rotational energies will be successively approximated.

Upon application of the change of variable $\mathrm{G}(R) \equiv R \mathrm{~F}(R)$, (3) becomes [17,27]

$$
-\frac{\hbar^{2}}{2 \mu} \mathrm{G}^{\prime \prime}(R)+\left[\frac{J(J+1) \hbar^{2}}{2 \mu R^{2}}+\mathrm{U}(R)-E\right] \mathrm{G}(R)=0,
$$

which cannot be solved without explicit knowledge of how $U$ varies with $R$ [28]. One way to overcome this obstacle is to expand $\mathrm{U}(R)$ as a Taylor series about $R_{e}$, the equilibrium internuclear separation:

$$
\mathrm{U}(R)=\mathrm{U}\left(R_{e}\right)+\mathrm{U}^{\prime}\left(R_{e}\right)\left(R-R_{e}\right)+\frac{\mathrm{U}^{\prime \prime}\left(R_{e}\right)\left(R-R_{e}\right)^{2}}{2 !}+\frac{\mathrm{U}^{\prime \prime \prime}\left(R_{e}\right)\left(R-R_{e}\right)^{3}}{3 !}+\cdots
$$

By definition, the potential energy has a global minimum at $R_{e}$, so $\mathrm{U}^{\prime}\left(R_{e}\right)=0$. Furthermore, the

reference point for potential energy is always arbitrary, and it is convenient to choose $\mathrm{U}\left(R_{e}\right)=0$. Under these conditions, Equation (5) becomes

$$
\mathrm{U}(R)=\frac{\mathrm{U}^{\prime \prime}\left(R_{e}\right)\left(R-R_{e}\right)^{2}}{2 !}+\frac{\mathrm{U}^{\prime \prime \prime}\left(R_{e}\right)\left(R-R_{e}\right)^{3}}{3 !}+\cdots,
$$

and the energy $E$ is now relative to $\mathrm{U}\left(R_{e}\right)=0$.

For low-lying vibrational levels $R \approx R_{e}$, and all terms in (6) save the first are small [17]. Neglecting these terms affords

$$
\mathrm{U}(R) \approx \frac{1}{2} k_{e} Q^{2},
$$

where $k_{e} \equiv \mathrm{U}^{\prime \prime}\left(R_{e}\right)$ and 


$$
Q \equiv R-R_{e}
$$

is the so-called normal coordinate. Equation (7), which is an approximate potential energy function for a diatomic molecule, is also a Hooke's law potential from classical mechanics. The constant $k_{e}$, called the equilibrium molecular force constant, is a measure of the "stiffness" of the diatomic bond and is completely analogous to the spring constant of classical physics [29].

In the approximation that a diatomic molecule vibrates like a one-dimensional harmonic oscillator, exact equality holds in (7). Under this assumption [and using Definition (8)], one may recast the differential equation (4) as

$$
-\frac{\hbar^{2}}{2 \mu} \Psi^{\prime \prime}(Q)+\left[\frac{J(J+1) \hbar^{2}}{2 \mu\left(R_{e}+Q\right)^{2}}+\frac{1}{2} k_{e} Q^{2}\right] \Psi(Q)=E \Psi(Q),
$$

where the change of variable $\Psi(Q) \equiv \mathrm{G}(R)$ was made [17] in order to convert to a coordinate system based on $Q$. The first bracketed term in (9) is the potential energy of rotation [27, 29] and is a result of the molecule's rotational angular momentum and concomitant centrifugal force field [28]; the second term in brackets is the potential energy of harmonic vibration. Equation (9) is merely the time-independent Schrödinger equation for a diatomic molecule undergoing real rotation and harmonic vibration.

One final simplifying assumption is necessary in order to solve (9). The molecule is conceptualized to undergo rigid rotation at a fixed internuclear separation $R_{e^{\circ}}$ In this "rigidrotator" approximation, the radial Schrödinger equation (9) becomes

$$
-\frac{\hbar^{2}}{2 \mu} \Psi^{\prime \prime}(Q)+\frac{1}{2} k_{e} Q^{2} \Psi(Q)=\left[E-\frac{J(J+1) \hbar^{2}}{2 \mu R_{e}^{2}}\right] \Psi(Q) .
$$

The rigid-rotator approximation is perhaps unsettling because this model precludes change in internuclear separation, yet the molecule has already been assumed to undergo harmonic vibration. Such philosophical difficulties are averted by expanding the centrifugal potential term in (9) as a Maclaurin series in $Q / R_{e}$. The base point for this expansion is $Q / R_{e}=0$, which corresponds by (8) to $R=R_{e}$. The explicit form of the series expansion is [17, 27]

$$
\begin{aligned}
\frac{J(J+1) \hbar^{2}}{2 \mu\left(R_{e}+Q\right)^{2}} & =\frac{J(J+1) \hbar^{2}}{2 \mu R_{e}^{2}} \frac{1}{\left(1+Q / R_{e}\right)^{2}} \\
& =\frac{J(J+1) \hbar^{2}}{2 I_{e}}\left[1-2 \frac{Q}{R_{e}}+3 \frac{Q^{2}}{R_{e}^{2}}-\cdots\right],
\end{aligned}
$$


where

$$
I_{e} \equiv \mu R_{e}^{2}
$$

is the equilibrium moment of inertia for a two-particle system. Note that the Taylor series (11) converges $[30,31]$ when

$$
\left|\frac{Q}{R_{e}}\right|<1,
$$

which by (8) is equivalent to $R<2 R_{e}$. If $R$ is within this radius of convergence, the magnitude of the terms in (11) must become successively smaller; neglecting all but the first term is equivalent to the rigid-rotator approximation discussed above.

Equation (10) does not contain a rotational potential energy term but in fact resembles the Schrödinger equation for a one-dimensional harmonic oscillator whose total energy has been diminished by a rotational term. Analysis [17, 27] of the boundary conditions of $\Psi(Q)$ shows that when $R \approx R_{e}$ (the assumption permeating this treatment), $\Psi$ may be represented by a (normalized) harmonic oscillator wave function,

$$
\Psi_{v}(Q)=\frac{1}{\left(2^{v} v !\right)^{\frac{1}{2}}}\left(\frac{\alpha}{\pi}\right)^{\frac{1}{4}} H_{v}(Q \sqrt{\alpha}) e^{-\frac{1}{2} Q^{2} \alpha},
$$

where $v=0,1,2, \ldots$ is the vibrational quantum number, $H_{v}$ is the $v$ th Hermite polynomial, and

$$
\alpha \equiv \frac{\mu v_{e}}{\hbar}
$$

The harmonic oscillator (14) vibrates sinusoidally about $R_{e}$ with a classical frequency $v_{e}$ given by

$$
v_{e}=\frac{1}{2 \pi} \sqrt{\frac{k_{e}}{\mu}} .
$$

The function $\Psi_{v}(Q)$ may be related back to the radial function ${ }^{3} F(R)$ and substituted into (2) to yield [17] an approximate diatomic vibro-rotational wave function:

$$
\psi_{v, J, M}^{(0)}=\frac{\Psi_{v}(Q)}{Q+R_{e}} Y_{J}^{M}(\theta, \phi)
$$

The subscript $N$ from (2) has been dropped in favor of the three quantum numbers on which $\psi$ depends. Furthermore, a superscript zero has been added to $\psi$ in anticipation of a perturbationtheoretical analysis of vibration and rotation; the wave function (17) for a harmonic oscillator/rigid-rotator provides a zeroth-order approximation to the true wave function for

\footnotetext{
${ }^{3}$ Recall the changes in variables made in Equations (4) and (9).
} 
internal nuclear motion.

\section{ANHARmonicity, Coupling, AND CENTRIFUgal Distortion}

Most molecules have a ${ }^{1} \Sigma$ ground electronic state, ${ }^{4}$ so at room temperature virtually all such molecules are in the ${ }^{1} \Sigma$ electron configuration. In this case, one may neglect electronic excitations, and vibro-rotational energy in the harmonic oscillator/rigid-rotator approximation is $[17,29]$

$$
E_{v, J}^{(0)}=\left(v+\frac{1}{2}\right) \omega_{e}+J(J+1) B_{e},
$$

where $\omega_{e} \equiv 2 \pi \hbar v_{e}, B_{e}$ is the equilibrium rotational constant,

$$
B_{e} \equiv \frac{\hbar^{2}}{2 I_{e}},
$$

and the superscript zero in (18) is analogous to the one in (17). Experimental values of $B_{e}$ and $\omega_{e}$ have been calculated (from spectroscopic data) and tabulated [32] for most diatomic molecules.

The energy expression (18) is strictly valid only for a diatomic molecule whose potential energy is given by $\mathrm{U}=\frac{1}{2} k_{e} Q^{2}$, which is the equation of a parabola. In reality, the potential energy curve for a diatomic molecule is not parabolic. Figure 1, for example, depicts accurate potential energy data for ${ }^{~} \mathrm{H}_{2}$ [33] obtained by solving the electronic Schrödinger equation; the harmonic oscillator potential is also plotted..$^{5}$ The most pronounced anharmonicity in $\mathrm{U}(Q)$ appears when $R$ is much larger than $R_{e}$ (that is, when $Q$ is much greater than zero), for while the quantum-mechanical harmonic oscillator has an infinite number of vibrational levels, the potential energy curve for a real dinuclear molecule asymptotically approaches the molecular dissociation energy as $Q$ increases, creating significant anharmonicity at large values of $Q$.

Because of anharmonicity in the potential energy curve, the average internuclear separation for a real diatomic molecule increases slightly with increasing $v$; this in turn increases the molecule's effective moment of inertia and therefore decreases its rotational energy [34]. The

\footnotetext{
${ }^{4}$ Molecules in the ${ }^{1} \Sigma$ state have zero net electronic orbital angular momentum and zero net electron spin. A few diatomic molecules do not have this ground state; these include $\mathrm{O}_{2}$, which has a ${ }^{3} \Sigma$ ground state, and all molecules possessing an odd number of electrons (e.g., $\mathrm{NO}, \mathrm{NO}_{2}$, and $\mathrm{ClO}_{2}$ ) [28].

${ }^{5}$ Note that there is no rotational potential energy in the rigid-rotator approximation, so the zeroth-order potential energy curve for a diatomic molecule is simply that of an harmonic oscillator.
} 
energy expression (18), however, fails to account for this coupling between vibration and rotation. ${ }^{6}$

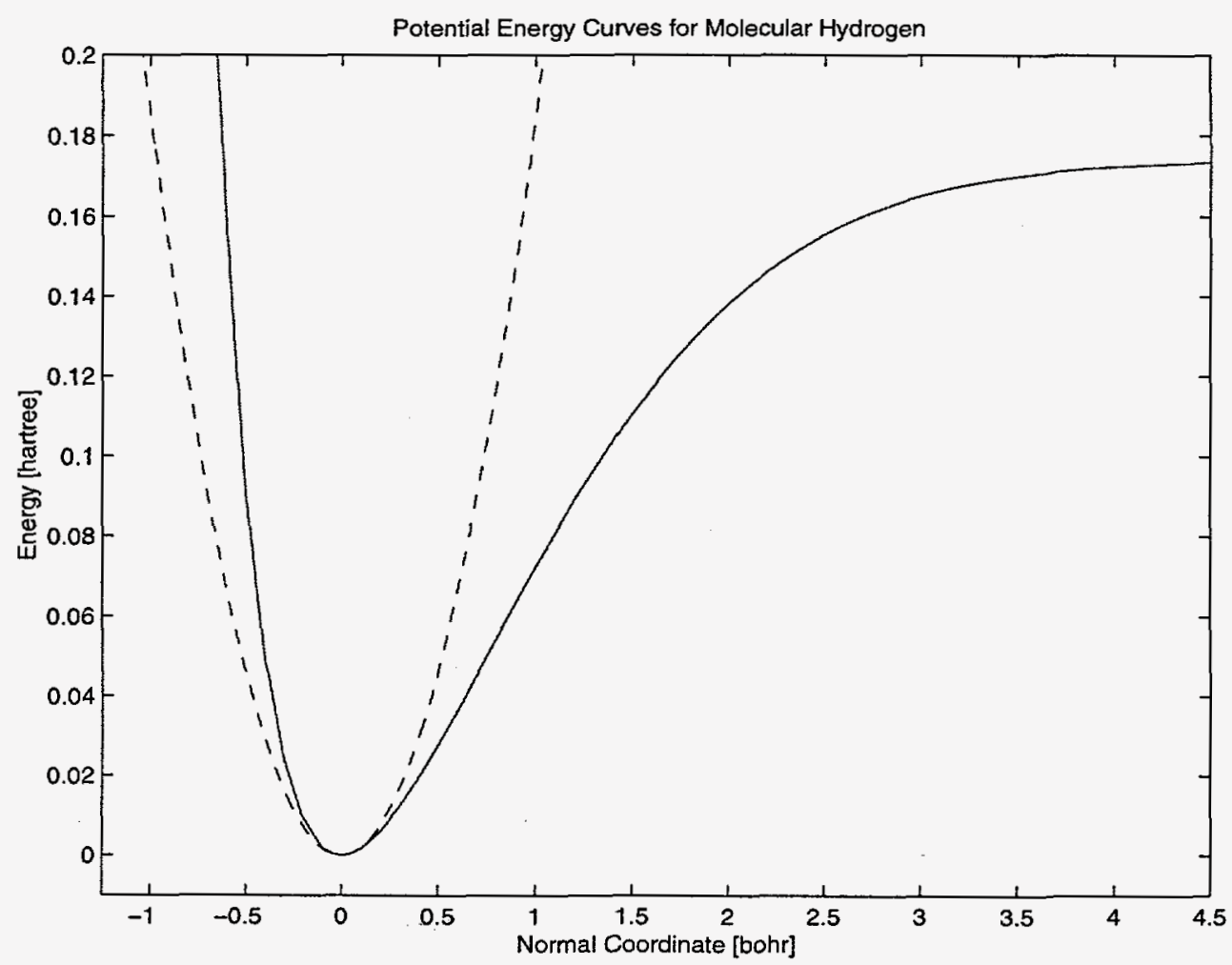

FIGURE 1. Real and harmonic potentials for $X^{1} \Sigma^{+}{ }_{g}{ }^{1} \mathrm{H}_{2}$. The "real" potential represents accurate data obtained [33] by solving the electronic Schrödinger equation using a 54 -term variational wave function with relativistic corrections. The requisite harmonic force constant $k_{e}$ was calculated by means of Equation (16) and the experimentally determined harmonic frequency $v_{e}$ given in [32].

Lastly, as a molecule's rotational energy increases, so too does its rate of rotation and hence its angular momentum. Since centrifugal force is proportional to angular momentum, an increase in rotational energy effectively stretches the molecule's bond against its restoring force [28], and as a result some energy is consumed in the form of work [34]. This phenomenon is known as centrifugal stretching or centrifugal distortion.

\footnotetext{
${ }^{6}$ As shown by Levine [17], vibrational-rotational coupling does not entirely disappear even in the limit of a perfectly parabolic potential well due to the increase in average moment of inertia that accompanies increasing $v$ and tends to decrease rotational energy.
} 


\section{Perturbation Theory for a Diatomic Molecule}

The harmonic oscillator/rigid-rotator wave functions (17) are exact solutions ${ }^{7}$ to the approximate Schrödinger equation (10). Equation (10) is an approximation to the true

Schrödinger equation because it incorporates a truncated potential operator, $\hat{V}_{\mathrm{HO}}$ :

$$
\hat{V}_{\mathrm{HO}}=\frac{1}{2} k_{e} Q^{2},
$$

the potential operator for an harmonic oscillator. Within the radii of convergence of the series

(6) and (11), the full potential energy operator $\hat{V}$ is represented by $\hat{V}_{\text {HO }}$ plus all of the terms in

(6) and (11) that were neglected in the course of the harmonic oscillator and rigid-rotator treatments. ${ }^{8}$ Thus,

$$
\hat{V}=\frac{1}{2} k_{e} Q^{2}+\sum_{i=1}^{\infty}\left[\frac{(-1)^{i}(i+1)(J+1) J B_{e}}{R_{e}^{i}} Q^{i}+\frac{k_{i+2}}{(i+2) !} Q^{i+2}\right],
$$

where Definition (19) was used and where the $j$ th-order force constant $k_{j}$ is defined as

$$
k_{j} \equiv \mathrm{U}^{(j)}\left(R_{e}\right)
$$

for all $j>2$. Some authors (e.g., Sprandel and Kern [8]) choose to incorporate the factorial terms from (21) into the force constants; however, Definition (22) provides a better analogy to the unperturbed case, since $k_{e}$ is merely a special case of (22) with $j=e=2$.

Successively higher-order corrections for anharmonicity, centrifugal distortion, and vibrational-rotational coupling are made by incorporating additional terms of the potential energy operator (21) into the approximate Schrödinger equation (10); these new terms manifest as perturbations to the harmonic oscillator/rigid-rotator Hamiltonian [17]. The addition of all terms in (21) provides the full Hamiltonian operator $\hat{\mathcal{H}}$ for internal nuclear motion:

$$
\hat{\mathcal{H}}=-\frac{\hbar^{2}}{2 \mu} \hat{\nabla}^{2}+\frac{1}{2} k_{e} Q^{2}+\sum_{i=1}^{\infty}\left[\frac{(-1)^{i}(i+1)(J+1) J B_{e}}{R_{e}^{i}} Q^{i}+\frac{k_{i+2}}{(i+2) !} Q^{i+2}\right],
$$

which again is exact only within the radii of convergence of series (6) and (11).

It is known [8] that the sequence of energy correction terms from perturbation theory is most likely to converge when the Hamiltonian is expanded as a power series with infinitely many separate perturbations. With this motivation, the Hamiltonian operator (23) is rewritten in the form

\footnotetext{
${ }^{7}$ To within the negligible difference in boundary conditions discussed in Section III.

${ }^{8}$ Note that the first term in series $(11), J(J+1) \hbar^{2} / 2 I_{e}$, does not appear as part of $\hat{V}$ or $\hat{V}_{\mathrm{HO}}$ because it is constant and is therefore subsumed into the system's eigenenergy [see Equation (10)].
} 


$$
\hat{\mathcal{H}}=\hat{\mathcal{H}}^{(0)}+\sum_{i=1}^{\infty} \hat{\mathcal{H}}^{(i)}
$$

where

$$
\hat{\mathcal{H}}^{(0)} \equiv \frac{-\hbar^{2}}{2 \mu} \hat{\nabla}^{2}+\frac{1}{2} k_{e} Q^{2}
$$

is the unperturbed Hamiltonian operator (corresponding to the harmonic oscillator/rigid-rotator system) and each $\hat{\mathcal{H}}^{(i)}$ is a perturbation.

The summations in (23) and (24) must be equal, yet there are numerous conceivable ways of constructing the perturbed Hamiltonian operators. When perturbation theory is applied through second order, the perturbations are traditionally written [27] as follows:

$$
\hat{\mathcal{H}}^{(1)} \equiv \frac{1}{6} k_{3} Q^{3}-\frac{2 J(J+1) B_{e}}{R_{e}} Q, \quad \hat{\mathcal{H}}^{(2)} \equiv \frac{1}{24} k_{4} Q^{4}+\frac{3 J(J+1) B_{e}}{R_{e}^{2}} Q^{2} .
$$

Here the first-order perturbation $\hat{\mathcal{H}}^{(1)}$ comprises both the first-order vibrational correction [that is, the cubic anharmonicity correction or the second term in series (6)] and the first-order rotational correction [the second term in series (11)]. Likewise, the second-order perturbation $\hat{\mathcal{H}}^{(2)}$ incorporates second-order potential energy corrections for both vibration and rotation. Extending this rationale to arbitrary order provides a convenient form for the perturbed Hamiltonian operators:

$$
\hat{\mathcal{H}}^{(i)} \equiv \frac{k_{i+2}}{(i+2) !} Q^{i+2}+\frac{(-1)^{i}(i+1)(J+1) J B_{e}}{R_{e}^{i}} Q^{i},
$$

or, equivalently,

$$
\hat{\mathcal{H}}^{(i)}=\frac{k_{i+2}}{(i+2) !} Q^{i+2}+\frac{(-1)^{i}(i+1) L^{2}}{2 I_{e}}\left(\frac{Q}{R_{e}}\right)^{i}
$$

since the angular momentum $\vec{L}$ of a rotating diatomic molecule has a magnitude $L$ given by

$$
L=\hbar \sqrt{J(J+1)} .
$$

The form of the perturbations in (28) parallels that used in second- [35] and fourth-order [36, 37] perturbation treatments of polyatomic molecules, in which each perturbed Hamiltonian is the sum of an anharmonicity correction and a rotational term containing momenta divided by moments or products of inertia.

The first term in each perturbation (27) comes from (6), and these terms correct for anharmonicity in the potential energy curve. The second part of (27) is a rotational perturbation, 
corresponding to a term in series (11). Rotational perturbations adjust the system's rotational angular momentum so as to account for centrifugal stretching effects [6]. Corrections for the coupling of vibration and rotation arise from integrals involving $\hat{\mathcal{H}}^{(i)}$; when these integrals are evaluated (see Section VI), the result is a function of $v$ multiplied by a constant involving $J$, the rotational quantum number. This results in coupled energy terms that depend on both the vibrational and rotational quantum numbers.

Using the general power series expansion (24) of the Hamiltonian operator, one may show $[9,26,38]$ that there exists an energy correction term $E_{v, J}^{(i)}$ and perturbed wave function $\psi_{v, J, M}^{(i)}$ associated with each perturbation $\hat{\mathcal{H}}^{(i)}$. These entities are related by the perturbation equations $[26,38]$, the $n$th of which is :

$$
\left(\hat{\mathcal{H}}^{(0)}-E_{v, J}^{(0)}\right) \psi_{v, J, M}^{(n)}=\sum_{i=1}^{n}\left(E_{v, J}^{(i)}-\hat{\mathcal{H}}^{(i)}\right) \psi_{v, J, M}^{(n-i)}
$$

When $n=0$, the system is unperturbed and Equation (30) reduces to the familiar Schrödinger equation for an unperturbed system:

$$
\hat{\mathcal{H}}^{(0)} \psi_{v, J, M}^{(0)}=E_{v, J}^{(0)} \psi_{v, J, M}^{(0)}
$$

The system's total energy $E_{v, J}$ and true wave function $\psi_{v, J, M}$ are the sums of their respective correction terms:

$$
\begin{gathered}
E_{v, J}=E_{v, J}^{(0)}+\sum_{i=1}^{\infty} E_{v, J}^{(i)}, \\
\psi_{v, J, M}=\psi_{v, J, M}^{(0)}+\sum_{i=1}^{\infty} \psi_{v, J, M}^{(i)} .
\end{gathered}
$$

\section{Evaluation of MATrix Elements}

To calculate energy corrections $E_{v, J}^{(i)}$ and perturbed wave functions $\psi_{v, J, M}^{(i)}$, one must evaluate numerous integrals of the form

$$
\mathcal{H}_{\xi_{1}, \xi_{2}}^{(z)} \equiv \int \psi_{\xi_{1}}^{(0) *} \hat{\mathcal{H}}^{(z)} \psi_{\xi_{2}}^{(0)} d \tau
$$

where the integral is taken over all configuration space $\tau$ and the ordered triple $\xi_{i}=\left(v_{i}, J_{i}, M_{i}\right)$ specifies the system's quantum state. The matrix element notation $\mathcal{H}_{\xi_{1}, \xi_{2}}^{(z)}$ introduced in (34) is 
somewhat nonstandard in that $\mathcal{H}_{\xi_{1}, \xi_{2}}^{(z)}$ involves zeroth-order wave functions rather than true wave functions, and the Hamiltonian operator is a perturbation.

Integrals such as (34) can, in general, be evaluated numerically; however, for the case of internal nuclear motion there exists [17] a simple procedure whereby an analytic solution may be obtained. Note that in spherical polar coordinates, the infinitesimal volume element $d \tau=R^{2} \sin \theta d R d \theta d \phi$, where $\theta$ and $\phi$ are the standard spherical polar angles [30]. Hence, in this coordinate system, the arbitrary Hamiltonian matrix element $\mathcal{H}_{\xi_{1}, \xi_{2}}^{(i)}$ becomes

$$
\begin{aligned}
\mathcal{H}_{\xi_{1}, \xi_{2}}^{(i)} & \equiv \iint_{R} \int_{\theta} \psi_{\phi}^{(0) *} \hat{\mathcal{H}}^{(i)} \psi_{\xi_{2}}^{(0)} R^{2} \sin \theta d \phi d \theta d R \\
& =\iint_{R} \int_{\theta} \Psi_{v_{1}}(Q) \hat{\mathcal{H}}^{(i)} \Psi_{v_{2}}(Q) Y_{J_{1}}^{M_{1}}(\theta, \phi) Y_{J_{2}}^{M_{2}}(\theta, \phi) \sin \theta d \phi d \theta d R,
\end{aligned}
$$

where the $R^{2}$ term disappears as a result of (8) and (17). Since $\Psi_{j}$ and $\hat{\mathcal{H}}^{(i)}$ both depend only on $Q$ [Equations (14) and (27), respectively] and $Q$ is a function of $R$ only [Equation (8)], these terms may be factored out of the $\phi$ and $\theta$ integrals:

$$
\begin{aligned}
\mathcal{H}_{\xi_{1}, \xi_{2}}^{(i)} & =\int_{0}^{\infty} \Psi_{v_{1}} \cdot \hat{\mathcal{H}}^{(i)} \Psi_{v_{2}} d R \iint_{\theta} Y_{J_{1}}^{M_{1}} Y_{J_{2}}^{M_{2}} \sin \theta d \phi d \theta \\
& =\int_{0}^{\infty}\left(\Psi_{v_{1}} \hat{\mathcal{H}}^{(i)} \Psi_{v_{2}} d R\right) \delta_{J_{1}, J_{2}} \delta_{M_{1}, M_{2}},
\end{aligned}
$$

where the Kronecker delta function $\delta$ arises from the orthonormality of the spherical harmonic functions [3].

As a final step, one uses Definitions (8) and (27) plus the linearity of $\hat{\mathcal{H}}$ to obtain

$$
\begin{aligned}
\mathcal{H}_{\xi_{1}, \xi_{2}}^{(i)} & =\left[\frac{k_{i+2}}{(i+2) !} \int_{-R_{e}}^{\infty} \Psi_{v_{1}} Q^{i+2} \Psi_{v_{2}} d Q+\frac{(-1)^{i}(i+1)(J+1) J B_{e}}{R_{e}^{i}} \int_{-R_{e}}^{\infty} \Psi_{v_{1}} Q^{i} \Psi_{v_{2}} d Q\right] \delta_{J_{1}, J_{2}} \delta_{M_{1}, M_{2}} \\
& =\left[\gamma_{\text {vib }}^{(i)}\left\langle Q^{i+2}\right\rangle_{v_{1}, v_{2}}+\gamma_{\text {rot }}^{(i)}\left\langle Q^{i}\right\rangle_{v_{1}, v_{2}}\right] \delta_{J_{1}, J_{2}} \delta_{M_{1}, M_{2}},
\end{aligned}
$$

where the normal coordinate matrix elements are defined [cf. Equation (34)] as

$$
\left\langle Q^{z}\right\rangle_{v, v^{\prime}} \equiv \int_{-\infty}^{\infty} \Psi_{v} Q^{z} \Psi_{v^{\prime}} d Q
$$


To a good approximation, the limits of integration in (37) and (38) may be interchanged; see [17] or [27] for discussion. The vibrational potential energy constants $\gamma_{\text {vib }}^{(i)}$ and rotational potential energy constants $\gamma_{\text {rot }}^{(i)}$ in (37) are simply the constant coefficients from (27):

$$
\gamma_{\mathrm{vib}}^{(i)} \equiv \frac{k_{i+2}}{(i+2) !}, \quad \gamma_{\mathrm{rot}}^{(i)} \equiv \frac{(-1)^{i}(i+1)(J+1) J B_{e}}{R_{e}^{i}}
$$

Because of the orthogonality of the spherical harmonic functions, the matrix element (37) is zero if $J_{1} \neq J_{2}$ or $M_{1} \neq M_{2}$. Thus, the only nontrivial Hamiltonian matrix elements are those of the form $\mathcal{H}_{\left(v_{1}, J_{1}, M_{1}\right),\left(v_{2}, J_{1}, M_{1}\right)}^{(i)}$, or $\mathcal{H}_{v_{1}, v_{2}}^{(i)}$ in less cumbersome notation. Note that the matrix element $\mathcal{H}_{v_{1}, v_{2}}^{(i)}$ contains an implicit dependence on the rotational quantum number $J$ insofar as $\gamma_{\text {rot }}^{(i)}$ depends upon $J$. Elimination of $J_{2}$ and $M_{2}$ reduces $\mathcal{H}^{(i)}$ from a sixth- to a second-rank tensor, which is simply a standard, two-dimensional matrix ${ }^{9}$ whose elements are given by

$$
\mathcal{H}_{v, v^{\prime}}^{(i)}=\gamma_{\text {vib }}^{(i)}\left\langle Q^{i+2}\right\rangle_{v, v^{\prime}}+\gamma_{\text {rot }}^{(i)}\left\langle Q^{i}\right\rangle_{v, v^{\prime}}
$$

The functions $\Psi_{v}$ and $\Psi_{v^{\prime}}$ in (38) are harmonic oscillator wave functions [see Equation (14)]. Working in the Heisenberg Lie algebra,${ }^{10}$ it is possible to show [39] that the normal coordinate integrals (38) form a vector space; moreover, using matrix multiplication, one can readily derive expressions for these integrals as functions of the quantum numbers $v$ and $v^{\prime}$ and the exponent $z$. However, an alternative approach yields better insight concerning the physical system.

The harmonic oscillator wave functions (14) incorporate the Hermite polynomials, which are related by the well-known recursive formula [6]

$$
x H_{n}(x)=n H_{n-1}(x)+\frac{1}{2} H_{n+1}(x) ;
$$

setting $x=Q \sqrt{\alpha}$ and $n=v$, this equation becomes

$$
Q \alpha^{\frac{1}{2}} H_{v}\left(Q \alpha^{\frac{1}{2}}\right)=v H_{v-1}\left(Q \alpha^{\frac{1}{2}}\right)+\frac{1}{2} H_{v+1}\left(Q \alpha^{\frac{1}{2}}\right) .
$$

By rearranging (14), one may express the Hermite polynomial $H_{v}\left(Q \alpha^{\frac{1}{2}}\right)$ in terms of a harmonic oscillator wave function:

$$
H_{v}\left(Q \alpha^{\frac{1}{2}}\right)=\Psi_{v}(Q)\left[\frac{\pi}{\alpha}\right]^{\frac{1}{4}}\left(2^{v} v !\right)^{\frac{1}{2}} e^{\frac{1}{2} Q^{2} \alpha}
$$

\footnotetext{
${ }^{9}$ Operator matrices such as $\mathcal{H}^{(i)}$ obey the same rules as matrices of numbers [17].

${ }^{10}$ The harmonic oscillator Hamiltonian is an element of the associative covering algebra for the Heisenberg Lie algebra [39].
} 
similar expressions can be obtained for $H_{v-1}$ and $H_{v+1}$ in terms of $\Psi_{v-1}$ and $\Psi_{v-1}$, respectively. Substituting these expressions for the Hermite polynomials in (42) leads to a great deal of cancellation and affords [17] the relatively simple expression

$$
Q \Psi_{v}=\left[\frac{v}{2 \alpha}\right]^{\frac{1}{2}} \Psi_{v-1}+\left[\frac{v+1}{2 \alpha}\right]^{\frac{1}{2}} \Psi_{v+1}
$$

Finally, one multiplies Equation (44) by $\Psi_{v^{\prime}}^{*}$ and integrates to obtain

$$
\begin{aligned}
\langle Q\rangle_{v^{\prime}, v} & =\left(\frac{v}{2 \alpha}\right)^{\frac{1}{2}} \int \Psi_{v^{\prime}}^{*} \Psi_{v-1} d \tau+\left(\frac{v+1}{2 \alpha}\right)^{\frac{1}{2}} \int \Psi_{v^{\prime}}^{*} \Psi_{v+1} d \tau \\
& =\left[\frac{v}{2 \alpha}\right]^{\frac{1}{2}} \delta_{v^{\prime}, v-1}+\left[\frac{v+1}{2 \alpha}\right]^{\frac{1}{2}} \delta_{v^{\prime}, v+1},
\end{aligned}
$$

where the orthonormality of the harmonic oscillator wave functions was applied. The physical insight is this: because the net overlap of distinct harmonic oscillator wave functions is zero, the allowed electric dipole transitions for $\Psi_{v}$ must be $\Delta v= \pm 1$ [29]. Real molecules, however, are not confined exclusively to these harmonic oscillator transitions, but exhibit additional transitions for $\Delta v= \pm 2, \pm 4, \ldots$ [34]. As expected, these selection rules arise mathematically from the perturbations $\hat{\mathcal{H}}^{(i)}$.

From Equation (37), the perturbed Hamiltonian matrix elements are, in general, functions of some power of the normal coordinate matrix, but Equation (45) deals only with $Q^{\prime}$. To obtain formulae for integrals analogous to (45) but involving higher powers of $\boldsymbol{Q}$, one employs matrix multiplication [17]; for example, the matrix $Q^{2}$ is simply the (matrix) product of two $Q$ matrices, ${ }^{11}$

$$
\left\langle Q^{2}\right\rangle_{v, v^{\prime}}=\sum_{j}\langle Q\rangle_{v, j}\langle Q\rangle_{j, v^{\prime}}
$$

where $j$ is a dummy index variable that is eliminated during the course of the computation. Explicit formulae for the normal coordinate matrix elements up to $\left\langle Q^{4}\right\rangle_{v, v}$, are provided by Wilson et al. [1].

\footnotetext{
"By including only their nonzero elements, these matrices can always be made square and of the same dimension. Thus, the matrix multiplication (46) is always defined.
} 


\section{RAYLEIGH-SCHRÖDINGER EXPANSIONS}

For vibrational-rotational analysis problems, it is convenient to use the RayleighSchrödinger form of perturbation theory, which is based on the assumption that the set of all unperturbed wave functions $\left\{\psi_{\xi}^{(0)}\right\}$ is a basis for the Hilbert space containing the true wave functions $\psi_{\xi}$ [26]. Thus, each perturbed wave function $\psi_{\xi}^{(n)}$ may be expressed as a linear combination of the wave functions in (17):

$$
\psi_{\xi}^{(n)}=\sum_{\xi^{\prime}} c_{\xi^{\prime}}^{(n)} \psi_{\xi^{\prime}}^{(0)}
$$

where $c_{\xi^{\prime}}^{(n)}$ is the $n$ th-order expansion coefficient associated with quantum state $\xi^{\prime}$. The summation in (47) runs over all possible values of the three quantum numbers $v, J$, and $M$.

Three useful results will greatly expedite calculation of the linear combination coefficients in (47). First, it is known [3,40] that the expansion coefficient $c_{v, J, M}^{(n)}$ does not affect the perturbation energy $E_{v, J}^{(n)}$, so one may set $c_{v, J, M}^{(n)}=0$ in the Rayleigh-Schrödinger expansion of $\psi_{v, J, M}^{(n)}$. If this is done, the expansion (47) simplifies to

$$
\psi_{\xi}^{(n)}=\sum_{\xi^{\prime} \neq \xi} c_{\xi^{\prime}}^{(n)} \psi_{\xi^{\prime}}^{(0)}
$$

Second, observe that

$$
\begin{aligned}
\int \psi_{\xi_{1}}^{(m) *} \hat{\mathcal{H}}^{(i)} \psi_{\xi_{2}}^{(n)} d \tau & =\sum_{\xi^{\prime} \neq \xi_{1}[}\left[c_{\xi^{\prime}}^{(m)} \sum_{\xi^{\prime \prime} \neq \xi_{2}} c_{\xi^{\prime \prime}}^{(n)} \int \psi_{\xi^{\prime}}^{(0) *} \hat{\mathcal{H}}^{(i)} \psi_{\xi^{\prime \prime}}^{(0)} d \tau\right] \\
& =\sum_{\xi^{\prime} \neq \xi_{1}}\left[c_{\xi^{\prime}}^{(m)} \sum_{\xi^{\prime \prime} \neq \xi_{2}} c_{\xi^{\prime}}^{(n)} \hat{\mathcal{H}}_{\xi^{\prime}, \xi^{\prime \prime}}^{(i)}\right],
\end{aligned}
$$

where the Rayleigh-Schrödinger expansion (48) was used. Similarly,

$$
\begin{aligned}
\int \psi_{\xi_{1}}^{(m) *} \psi_{\xi_{2}}^{(n)} d \tau & =\sum_{\xi^{\prime} \neq \xi_{1}}\left[c_{\xi^{\prime}}^{(m)} \sum_{\xi^{\prime \prime} \neq \xi_{2}} c_{\xi^{\prime \prime}}^{(n)} \int \psi_{\xi^{\prime}}^{(0) *} \psi_{\xi^{\prime \prime}}^{(0)} d \tau\right] \\
& =\sum_{\xi^{\prime} \neq \xi_{1}}\left[c_{\xi^{\prime}}^{(m)} \sum_{\xi^{\prime \prime} \neq \xi_{2}} c_{\xi^{\prime \prime}}^{(n)} \delta_{\xi^{\prime}, \xi^{\prime \prime}}\right] .
\end{aligned}
$$


The second equality in (50) follows from the fact that the zeroth-order wave functions are orthonormal. ${ }^{12}$

If either of $m$ or $n$ is zero in (49) or (50), then one or both of the wave functions does not need to be expanded. These formulae are still valid, however, provided one defines

$$
c_{\xi^{\prime}}^{(0)} \equiv \delta_{\xi, \xi^{\prime}}
$$

where $\xi$ is the quantum state whose wave function is to be expanded and $\xi^{\prime}$ is the index variable of the Rayleigh-Schrödinger expansion (48). Definition (51) simply means that a zeroth-order wave function is a linear combination of a single wave function, namely, itself.

Using the results obtained above, one can derive a general formula for the RayleighSchrödinger expansion coefficients from the perturbation equations. Applying (48) and the notation introduced in this section, one obtains for the $n$th perturbation equation (30)

$$
\sum_{\xi^{\prime \prime} \neq \xi} c_{\xi^{\prime \prime}}^{(n)}\left(\hat{\mathcal{H}}^{(0)}-E_{v, J}^{(0)}\right) \psi_{\xi^{\prime \prime}}^{(0)}=\sum_{i=1}^{n}\left(E_{v, J}^{(i)}-\hat{\mathcal{H}}^{(i)}\right) \psi_{\xi}^{(n-i)}
$$

Since the coefficients for $n=0$ are known [from (51)], let $n$ be greater than zero. Pre-multiplying (52) by the complex conjugate $\psi_{\xi^{\prime}}^{(0) *}$ of an arbitrary state function and integrating over all space, one obtains

$$
\sum_{\xi^{\prime \prime \neq \xi}} c_{\xi^{\prime}}^{(n)} \int \psi_{\xi^{\prime}}^{(0) *}\left(\hat{\mathcal{H}}^{(0)}-E_{v, J}^{(0)}\right) \psi_{\xi^{\prime}}^{(0)} d \tau=\sum_{i=1}^{n} \int \psi_{\xi^{\prime}}^{(0) *}\left(E_{v, J}^{(i)}-\hat{\mathcal{H}}^{(i)}\right) \psi_{\xi}^{(n-i)} d \tau,
$$

which can be simplified by using the unperturbed Schrödinger equation (31) to perform the operation $\hat{\mathcal{H}}^{(0)} \psi_{\xi^{\prime \prime}}^{(0)}$ :

$$
\sum_{\xi^{\prime \prime} \neq \xi} c_{\xi^{\prime \prime}}^{(n)}\left(E_{v^{\prime \prime}, J^{\prime \prime}}^{(0)}-E_{v, J}^{(0)}\right) \delta_{\xi^{\prime}, \xi^{\prime \prime}}=\sum_{i=1}^{n}\left(E_{v, J}^{(i)} \int \psi_{\xi^{\prime}}^{(0) *} \psi_{\xi}^{(n-i)} d \tau\right)-\sum_{i=1}^{n} \int \psi_{\xi^{\prime}}^{(0) *} \hat{\mathcal{H}}^{(i)} \psi_{\xi}^{(n-i)} d \tau
$$

As $\xi^{\prime}$ is arbitrary, choose $\xi^{\prime}$ such that $\xi^{\prime} \neq \xi$. Under this condition, the wave functions $\psi_{\xi^{\prime}}^{(0)}$ and $\psi_{\xi}^{(n-i)}$ are not necessarily orthogonal [26]; however, in the case where $i=n$, these two functions are orthogonal, so (54) reduces to

$$
c_{\xi^{\prime}}^{(n)}\left(E_{v^{\prime}, J^{\prime}}^{(0)}-E_{v, J}^{(0)}\right)=\sum_{i=1}^{n-1}\left(E_{v, J}^{(i)} \int \psi_{\xi^{\prime}}^{(0) *} \psi_{\xi}^{(n-i)} d \tau\right)-\sum_{i=1}^{n} \int \psi_{\xi^{\prime}}^{(0) *} \hat{\mathcal{H}}^{(i)} \psi_{\xi}^{(n-i)} d \tau
$$

Applying (50-52), Equation (55) becomes

\footnotetext{
${ }^{12}$ These wave functions are (or can be chosen to be) orthogonal because they are eigenfunctions of a Hermitian operator, in this case $\hat{\mathcal{H}}^{(0)}$; the normalization condition is trivial.
} 


$$
c_{\xi^{\prime}}^{(n)}\left(E_{v^{\prime}, J^{\prime}}^{(0)}-E_{v, J}^{(0)}\right)=\sum_{i=1}^{n-1} c_{\xi^{\prime}}^{(n-i)} E_{v, J}^{(i)}-\sum_{i=1}^{n} \sum_{\xi^{\prime \prime} \neq \xi} c_{\xi^{\prime \prime}}^{(n-i)} \mathcal{H}_{\xi^{\prime}, \xi^{\prime \prime}}^{(i)}
$$

for all $n>0$, which is nearly the desired general formula. However, if $\xi$ and $\xi^{\prime}$ are degenerate quantum states with the same energy in the harmonic oscillator/rigid-rotator approximation, then the left side of (56) is zero, and no information regarding the expansion coefficients can be obtained from this formulation. Hence, assume for the moment that $E_{v^{\prime}, J^{\prime}}^{(0)} \neq E_{v, J}^{(0)}$.

Solving (56) for $c_{\xi^{\prime}}^{(n)}$ and substituting this expression into (48) affords the expansion

$$
\psi_{\xi}^{(n)}=\sum_{\xi^{\prime} \neq \xi}\left[\frac{1}{E_{v^{\prime}, J^{\prime}}^{(0)}-E_{v, J}^{(0)}}\left(\sum_{i=1}^{n-1} c_{\xi^{\prime}}^{(n-i)} E_{v, J}^{(i)}-\sum_{i=1}^{n} \sum_{\xi^{\prime \prime} \neq \xi} c_{\xi^{\prime \prime}}^{(n-i)} \mathcal{H}_{\xi^{\prime}, \xi^{\prime \prime}}^{(i)}\right) \psi_{\xi^{\prime}}^{(0)}\right]
$$

Recall from the preceding section that the only nontrivial Hamiltonian matrix elements $\mathcal{H}_{\xi^{\prime}, \xi^{\prime \prime}}^{(i)}$ are those for which $J^{\prime}=J^{\prime \prime}$ and $M^{\prime}=M^{\prime \prime}$. Furthermore, observe from (56) with $n=1$ that each first-order expansion coefficient $c_{\xi^{\prime}}^{(1)}$ is simply a Hamiltonian matrix element divided by an energy difference. By induction on $n$, one may show that every set of $n$ th-order expansion coefficients $\left\{c_{\xi^{\prime}}^{(n)}\right\}$ is nothing more than a sum of such terms, some of which are multiplied by an energy correction $E_{v, J}^{(i)}$. Thus, the entire right side of (57) is zero whenever $J^{\prime} \neq J^{\prime \prime}$ or $M^{\prime} \neq M^{\prime \prime}$, so the summations over $\xi^{\prime} \neq \xi$ and $\xi^{\prime \prime} \neq \xi$ in (56) and (57) reduce to summations over $v^{\prime} \neq v$ and $v^{\prime \prime} \neq v$, respectively. Applying this simplification and making use of (51), one may also write Equation (56) as

$$
c_{\xi^{\prime}}^{(n)}\left(E_{v^{\prime}, J^{\prime}}^{(0)}-E_{v, J}^{(0)}\right)=-\mathcal{H}_{v, v^{\prime}}^{(n)}+\sum_{i=1}^{n-1} c_{\xi^{\prime}}^{(n-i)} E_{v, J}^{(i)}-\sum_{i=1}^{n-1} \sum_{v^{\prime \prime} \neq \nu} c_{\xi^{\prime \prime}}^{(n-i)} \mathcal{H}_{v^{\prime}, v^{\prime \prime}}^{(i)} .
$$

In obtaining Equations (57) and (58), it was assumed that $E_{v^{\prime}, J^{\prime}}^{(0)} \neq E_{v, J}^{(0)}$. In general, the difference in zeroth-order energies between states $\xi^{\prime}$ and $\xi$ is

$$
E_{v^{\prime}, J^{\prime}}^{(0)}-E_{v, J}^{(0)}=\left(v^{\prime}-v\right) \omega_{e}+\left(J^{\prime}-J\right)\left(J^{\prime}+J+1\right) B_{e}
$$

from (18). However, the outer summation in the final Rayleigh-Schrödinger expansion (57) runs over only quantum numbers $v^{\prime} \neq v$ and therefore $J=J^{\prime}$ and $M=M^{\prime}$. Under these conditions, the energy difference (59) reduces to

$$
E_{v^{\prime}, J^{\prime}}^{(0)}-E_{v, J}^{(0)}=\left(v^{\prime}-v\right) \omega_{e} .
$$


Hence, the zeroth-order energy differences are independent of the rotational quantum number. Moreover, since (58) need contain no summations over $J$ or $M$, the rotational quantum number $J$ appears in this equation only as a multiplicative constant [recall Definition (39) of the $i$ th-order rotational potential energy constant]; the quantum number $M$ does not appear in (58) at all. Because of this, the Rayleigh-Schrödinger expansion coefficients $c_{\xi^{\prime}}^{(n)}$ in (58) will be denoted instead by $c_{v^{\prime}}^{(n)}$, where an implicit parametric dependence on $J$ (analogous to that of $\mathcal{H}_{v, v^{\prime}}^{(i)}$ ) is assumed.

Equation (60) is significant because it implies that $E_{v^{\prime}, J^{\prime}}^{(0)}-E_{v, J}^{(0)} \neq 0$ if $v^{\prime} \neq v$. Since Equation (58) relates to the Rayleigh-Schrödinger expansion

$$
\psi_{v, J, M}^{(n)}=\sum_{v^{\prime} \neq v} c_{v^{\prime}}^{(n)} \psi_{v^{\prime}, J, M}^{(0)}
$$

this condition is met, so the assumption that $E_{v^{\prime}, J^{\prime}}^{(0)} \neq E_{v, J}^{(0)}$ is justified. The final expression for the Rayleigh-Schrödinger coefficients is obtained from (58):

$$
c_{v^{\prime}}^{(n)}=\frac{1}{\left(v-v^{\prime}\right) h v_{e}}\left[\mathcal{H}_{v, v^{\prime}}^{(n)}-\sum_{i=1}^{n-1} c_{v^{\prime}}^{(n-i)} E_{v, J}^{(i)}+\sum_{i=1}^{n-1} \sum_{v^{\prime \prime} \neq v} c_{v^{\prime \prime}}^{(n-i)} \mathcal{H}_{v^{\prime}, v^{\prime \prime}}^{(i)}\right],
$$

where (60) was used.

Equation (62) provides an important recursive relation whereby each new set of

expansion coefficients $\left\{c_{v^{\prime}}^{(n)}\right\}$ is determined by all of the coefficients of order less than $n$.

Reference to molecular vibrations and rotations was made only in the context of obtaining a value for $E_{v^{\prime}, J^{\prime}}^{(0)}-E_{v, J}^{(0)}$, so the remainder of the derivation is valid for any Rayleigh-Schrödinger perturbation problem involving a power series expansion of the Hamiltonian.

\section{Energy Corrections}

Equations (61) and (62) are necessary in order to expand the perturbed wave function 
$\psi_{\xi}^{(i)}$ in terms of the known functions in the set $\left\{\psi_{\xi^{\prime}}^{(0)}\right\}{ }^{13}$ Such perturbed wave functions appear in the equation for the $n$ th-order energy correction [26],

$$
\begin{aligned}
E_{v, J}^{(n)}=\sum_{j=1}^{\kappa} \sum_{i=j}^{\kappa} & {\left[\left(2-\delta_{n, 2 i}\right\rangle\left\langle\psi_{\xi}^{(j-1)}\left|\hat{\mathcal{H}}^{(n-i-j+1)}\right| \psi_{\xi}^{(i)}\right\rangle\right]+\sum_{i=0}^{\kappa-1}\left\langle\psi_{\xi}^{(i)}\left|\hat{\mathcal{H}}^{(n-2 i)}\right| \psi_{\xi}^{(i)}\right\rangle } \\
& -\sum_{j=2}^{\kappa} \sum_{i=j}^{\kappa}\left[\left(2-\delta_{n, 2 i}\right) E_{v, J}^{(n-i-j+1)}\left\langle\psi_{\xi}^{(j-1)} \mid \psi_{\xi}^{(i)}\right\rangle\right]-\sum_{i=1}^{\kappa-1} E_{v, J}^{(n-2 i)}\left\langle\psi_{\xi}^{(i)} \mid \psi_{\xi}^{(i)}\right\rangle \\
+ & {\left[\left\langle\psi_{\xi}^{(\kappa)}\left|\hat{\mathcal{H}}^{(1)}\right| \psi_{\xi}^{(\kappa)}\right\rangle-E_{v, J}^{(1)}\left\langle\psi_{\xi}^{(\kappa)} \mid \psi_{\xi}^{(\kappa)}\right\rangle\left(1-\delta_{n, 1}\right)\right] \delta_{n, 2 \kappa+1}, }
\end{aligned}
$$

where Dirac bra-ket notation has been introduced; that is

$$
\left\langle\psi_{\xi_{1}}^{(i)} \mid \psi_{\xi_{2}}^{(j)}\right\rangle \equiv \int \psi_{\xi_{1}}^{(i) *} \psi_{\xi_{2}}^{(j)} d \tau
$$

and

$$
\left\langle\psi_{\xi_{1}}^{(i)}\left|\hat{\mathcal{H}}^{(z)}\right| \psi_{\xi_{2}}^{(j)}\right\rangle \equiv \int \psi_{\xi_{1}}^{(i) *} \hat{\mathcal{H}}^{(z)} \psi_{\xi_{2}}^{(j)} d \tau
$$

The parameter $\kappa$ in $(63)$ is defined as

$$
\kappa \equiv\left\lfloor\frac{1}{2} n\right\rfloor,
$$

the greatest integer less than or equal to $\frac{1}{2} n$. Mathematically, only the wave function terms $\psi_{\xi}^{(0)}, \psi_{\xi}^{(1)}, \ldots, \psi_{\xi}^{(\kappa)}$ are necessary to express the $n$ th-order energy correction $E_{v, J}^{(n)}[40]$.

Using Equations (48-50) to expand the wave functions in (63) provides

\footnotetext{
${ }^{13}$ A nagging problem throughout this discussion is whether to include as subscripts the quantum numbers $J$ and $M$. The results of Section VI justify omission of $M$ as a subscript for $c^{(n)}$ because Hamiltonian matrix elements do not depend on $M$, nor is $M$ a necessary summation variable in the Rayleigh-Schrödinger expansion (61). Likewise, $J$ is not a necessary summation variable, although it does appear in Hamiltonian matrix elements as part of the rotational potential energy constants of (39). A subscript $J$ is not included on Hamiltonian matrix elements, however, in order to emphasize that $\mathcal{H}^{(i)}$ is a (two-dimensional) matrix whose indices are vibrational quantum numbers, whereas $J$ appears only in the aforementioned rotational potential energy constants. In keeping with this convention, the $J$ subscript of the expansion coefficients is suppressed. Whenever a wave function explicitly appears in a formula, however, all three quantum numbers $v, J$, and $M$ will be retained (often as the single subscript $\xi$ ) until an integration or other operation is performed that formally removes dependence on $J$ and $M$. Furthermore, the energy corrections $E^{(i)}$ will retain both $v$ and $J$ as subscripts in order to emphasize the presence of both vibrational and rotational energy levels.
} 


$$
\begin{aligned}
E_{v, J}^{(n)}=\sum_{j=1}^{\kappa} \sum_{i=j}^{\kappa}[(2 & \left.\left.-\delta_{n, 2 i}\right) \sum_{v^{\prime} \neq v}\left(c_{v^{\prime}}^{(j-1)} \sum_{v^{\prime \prime} \neq v} c_{v^{\prime}}^{(i)} \mathcal{H}_{v^{\prime}, v^{\prime \prime}}^{(n-i+1)}\right)\right]+\sum_{i=0}^{\kappa-1} \sum_{v^{\prime} \neq v}\left(c_{v^{\prime}}^{(i)} \sum_{v^{\prime \prime} \neq v} c_{v^{\prime \prime}}^{(i)} \mathcal{H}_{v^{\prime}, v^{\prime \prime}}^{(n-2 i)}\right) \\
& -\sum_{j=2}^{\kappa} \sum_{i=j}^{\kappa}\left[\left(2-\delta_{n, 2 i}\right) E_{v, J}^{(n-i-j+1)} \sum_{v^{\prime} \neq v}\left(c_{v^{\prime}}^{(j-1)} \sum_{v^{\prime \prime} \neq v} c_{v^{\prime \prime}}^{(i)} \delta_{v^{\prime}, v^{\prime \prime}}\right)\right] \\
& -\sum_{i=1}^{\kappa-1}\left[E_{v, J}^{(n-2 i)} \sum_{v^{\prime} \neq v}\left(c_{v^{\prime}}^{(i)} \sum_{v^{\prime \prime} \neq v} c_{v^{\prime \prime}}^{(i)} \delta_{v^{\prime}, v^{\prime \prime}}\right)\right] \\
& +\left[\sum_{v^{\prime} \neq v}\left(c_{v^{\prime}}^{(\kappa)} \sum_{v^{\prime \prime} \neq v} c_{v^{\prime \prime}}^{(\kappa)} \mathcal{H}_{v^{\prime}, v^{\prime \prime}}^{(1)}\right)-\left(1-\delta_{n, 1}\right) E_{v, J}^{(1)} \sum_{v^{\prime} \neq v}\left(c_{v^{\prime}}^{(\kappa)} \sum_{v^{\prime \prime} \neq v} c_{v^{\prime \prime}}^{(\kappa)} \delta_{v^{\prime}, v^{\prime \prime}}\right)\right] \delta_{n, 2 \kappa+1} .
\end{aligned}
$$

Hamiltonian matrix elements in (67) may be converted to normal coordinate matrix elements by using Equation (40), and Kronecker delta functions of the form $\delta_{v^{\prime}, v^{\prime \prime}}$ simplify some of the nested summations in (67), leaving

$$
\begin{aligned}
E_{v, J}^{(n)}=\sum_{\substack{\chi=0, \chi=2}}\left\{\sum_{j=1}^{\kappa}\right. & \sum_{i=j}^{\kappa}\left[\gamma_{\chi}^{(n-i-j+1)}\left(2-\delta_{n, 2 i}\right) \sum_{v^{\prime} \neq v}\left(c_{v^{\prime}}^{(j-1)} \sum_{v^{\prime \prime} \neq v} c_{v^{\prime \prime}}^{(i)}\left\langle Q^{n-i-j+1+\chi}\right\rangle_{v^{\prime}, v^{\prime \prime}}\right)\right] \\
& +\sum_{i=0}^{\kappa-1}\left[\gamma_{\chi}^{(n-2 i)} \sum_{v^{\prime} \neq v}\left(c_{v^{\prime}}^{(i)} \sum_{v^{\prime \prime} \neq v} c_{v^{\prime \prime}}^{(i)}\left\langle Q^{n-2 i+\chi}\right\rangle_{v^{\prime}, v^{\prime \prime}}\right)\right] \\
& -\sum_{j=2}^{\kappa} \sum_{i=j}^{\kappa}\left[\left(2-\delta_{n, 2 i}\right) E_{v, J}^{(n-i-j+1)} \sum_{v^{\prime} \neq v}\left(c_{v^{\prime}}^{(j-1)} c_{v^{\prime}}^{(i)}\right)\right]-\sum_{i=1}^{\kappa-1}\left[E_{v, J}^{(n-2 i)} \sum_{v^{\prime} \neq v}\left(c_{v^{\prime}}^{(i)}\right)^{2}\right] \\
& \left.+\left[\gamma_{\chi}^{(1)} \sum_{v^{\prime} \neq v}\left(c_{v^{\prime}}^{(\kappa)} \sum_{v^{\prime \prime} \neq v} c_{v^{\prime \prime}}^{(\kappa)}\left\langle Q^{1+\chi}\right\rangle_{v^{\prime}, v^{\prime \prime}}\right)-\left(1-\delta_{n, 1}\right) E_{v, J}^{(1)} \sum_{v^{\prime} \neq v}\left(c_{v^{\prime}}^{(\kappa)}\right)^{2}\right] \delta_{n, 2 x+1}\right\} .
\end{aligned}
$$

The outermost index variable $\chi$ in (68) assumes only two values, 0 or 2 , with

$$
\gamma_{0}^{(z)} \equiv \gamma_{\text {rot }}^{(z)}, \quad \gamma_{2}^{(z)} \equiv \gamma_{\text {vib }}^{(z)}
$$

This amounts to evaluating Equation (68) once for rotational perturbations and once for vibrational perturbations.

Expressed in terms of normal coordinate matrix elements, the Rayleigh-Schrödinger coefficients in (68) have the form

$$
c_{v^{\prime}}^{(n)}=\sum_{\substack{\chi=0 \\ \chi=2}}\left[\frac{1}{\left(v-v^{\prime}\right) h v_{e}}\left(\gamma_{\chi}^{(n)}\left\langle Q^{n+\chi}\right\rangle_{v, v^{\prime}}-\sum_{i=1}^{n-1} c_{v^{\prime}}^{(n-i)} E_{v, J}^{(i)}+\sum_{i=1}^{n-1} \gamma_{\chi}^{(i)} \sum_{v^{\prime \prime} \neq v} c_{v^{\prime \prime}}^{(n-i)}\left\langle Q^{i+\chi}\right\rangle_{v^{\prime}, v^{\prime \prime}}\right)\right]
$$

where (40), (62), and (69) were used.

Evaluation of summations over quantum states such as those in (68) and (70) has traditionally been one of the foremost difficulties encountered in any application of perturbation theory $[17,40]$; however, in this context such is not the case, for the summations over $v^{\prime} \neq v$, etc., in (68) and (70) are not infinite sums. Rather, because there are only a finite number of allowed 
transitions and since normal coordinate matrix elements must vanish for forbidden transitions, $\left\langle Q^{z}\right\rangle_{i, j}$ turns out to be zero whenever $i \neq j-z, j-z+2, \ldots, j+z-2, j+z$. Thus, this matrix element consists of no more than $z+1$ nonzero terms. This result is one reason that RayleighSchrödinger perturbation theory is so often used in vibrational-rotational analysis.

The above selection rules for the elements of the matrix $Q^{z}$ arise from the fact that the harmonic oscillator wave function $\Psi_{v}$ has a definite parity [3] corresponding to the parity of the vibrational quantum number $v$. Furthermore, $Q^{z}$ in $\left\langle Q^{z}\right\rangle_{i, j}$ has the same parity ${ }^{14}$ as the integer $z$, so the parity of $\Psi_{i}^{*} Q^{z} \Psi_{j}$ is the same as that of the integer $z+i+j$. As the integral of an odd function over all space is zero, $\left\langle Q^{z}\right\rangle_{i, j}=0$ whenever $z+i+j$ is odd, or in other words when $i \neq j-z, j-z+2, \ldots, j+z-2, j+z$.

\section{IMPLEMENTATION USING MATHEMATICA}

An existing Mathematica code [9] can evaluate the matrices $Q^{z}$ for any positive integer $z$ and return analytic functions of the vibrational quantum number analogous to (45); the selection rules for these matrices (as discussed above) are $\Delta v= \pm 1, \pm 3, \pm 5, \ldots, \pm z$ when $z$ is odd and $\Delta v=0, \pm 2, \pm 4, \ldots, \pm z$ when $z$ is even. This Mathematica code, along with Equations (68) and (70), could in principle be used by Mathematica to derive arbitrary-order correction terms. However, Mathematica itself is capable of performing the algebra necessary to transform the general energy expression (63) into Equation (68). Hence, in the interest of maximum versatility, the general expressions (63) for the energy corrections and (58) for the Rayleigh-Schrödinger coefficients were used instead of (68) and (70). The advantage of this approach is that the perturbed Hamiltonian operators and zeroth-order energy differences are defined by means of short, readily alterable functions that are separate from the main Mathematica code. As such, these definitions can be changed quickly should one wish to employ the Rayleigh-Schrödinger perturbation framework to solve a quantum-mechanical problem other than the one discussed herein.

Appendices A and B provide the complete Mathematica code necessary to derive analytic expressions for $E_{v, J}^{(1)}, E_{v, J}^{(2)}, \ldots$ in terms of molecular constants, universal constants, and

\footnotetext{
${ }^{14}$ Recall that $Q$ is the variable of integration in $\left\langle Q^{z}\right\rangle_{i, j}$, and not simply some number. Therefore, in this context $Q^{z}$ is a function with definite parity.
} 
quantum numbers. Appendix A defines a Mathematica package called RSPERTURB that contains a slightly modified version of the code of Dudas et al. [9] plus Equations (48-50), (58), and (63), along with a few other assorted rules for manipulating quantum-mechanical matrix elements. The code in this package pertains to Rayleigh-Schrödinger perturbation theory in general. Appendix B, on the other hand, defines the package DIATOMICVIBRoT, which consists of Definition (60) of $E_{v^{\prime}, J^{\prime}}^{(0)}-E_{v, J}^{(0)}$ and Definition (27) of $\hat{\mathcal{H}}^{(i)}$; the purpose of this Mathematica package is to apply the functions in RSPERTURB to the vibrational-rotational analysis of diatomic molecules.

In deriving energy formulae using RSPERTURB and DIATOMICVIBROT, the quantum number $v$ was not incorporated symbolically, but instead separate energy expressions were derived for each value of $v$. There are several reasons why this was done. First and foremost, when $v$ is known explicitly, the summations over quantum numbers $v^{\prime} \neq v$, etc., may be quickly evaluated, so it is enormously simpler (and much more efficient) to derive formulae in this manner.

Moreover, the perturbation analysis described here is accurate only when $R \approx R_{e}$ (that is, when $v$ is small), so there are relatively few vibrational states for which this analysis is applicable (and hence for which energy formulae need be derived).

The only reason one might desire a general energy expression in terms of both $v$ and $J$ is that such an expression could subsequently be factored into a polynomial in powers of $J(J+1)$ and $\left(v+\frac{1}{2}\right)$ whose coefficients could be related to the important spectroscopic constants, as shown by Dunham [41]. When only first- and second-order energy corrections are included, such a procedure has been utilized [17] to derive $a b$ initio formulae for the spectroscopic constants in terms of universal constants, molecular constants, and the quantum numbers $v$ and $J$. In general, however, perturbation calculations do not yield energies that can be factored exactly (i.e., analytically) into powers of $J(J+1)$ and $\left(v+\frac{1}{2}\right)$ [42]. Furthermore, few of the Dunham coefficients have been attributed any physical or spectroscopic meaning $[28,43]$, and indeed the Dunham expansion in $\left(v+\frac{1}{2}\right)^{i}[J(J+1)]^{j}$ is most often used as a numerical fitting equation. In light of this, the most efficient way to obtain theoretical values for spectroscopic constants is to calculate vibrational and rotational energy levels from first principles, then numerically fit these values to an appropriate power series in much the same way that $a b$ initio electronic energies are fit to an analytic potential energy function. 
Using the external packages RSPERTURB and DIATOMICVIBROT, Mathematica $^{15}$ derived analytic formulae for the energy correction terms $E^{(1)}$ through $E^{(6)}$ and for vibrational states $v=0$ through $v=10$. The odd-order perturbation energies were found to be zero, owing to the fact that perturbed Hamiltonian matrix elements $\mathcal{H}_{v_{1}, v_{2}}^{(i)}$ have a definite parity due to Definition (27) and the parity of $\left\langle Q^{z}\right\rangle_{v_{1}, v_{2}}$ (as discussed in the preceding section). Using intrinsic Mathematica functions for algebraic simplification, ${ }^{16}$ one can express each correction formula as a linear combination of small terms; the linear combination coefficients are integers whose values depend upon the vibrational state. By taking advantage of linear combination notation and intrinsic patterns in the correction formulae, one can reduce these expressions from literally hundreds of pages of algebra into relatively compact forms. These compact expressions for the energy corrections $E^{(2)}, E^{(4)}$, and $E^{(6)}$ are given in Appendices C-E.

Each energy correction formula consists of purely vibrational terms, purely rotational terms, and coupling terms arising from the interaction of vibration and rotation. Terms containing rotational dependence of some description are easily identified by the presence of the quantum number $J$, while purely vibrational contributions are conspicuous by the absence of this factor. Coupling terms are distinguishable from purely rotational contributions on the basis of their integer coefficients: the coefficients for a purely rotational contribution to the energy will not change with $v$, and such terms can therefore be grouped together into a single term for pure rotation (see Appendix C).

The procedure used to derive $E^{(2)}, E^{(4)}$, and $E^{(6)}$ is completely general and works for arbitrarily high orders of correction; the maximum order of correction is potentially limited only by time constraints. Previously, researchers using Mathematica to solve problems in quantum chemistry have reported [44] that this software is perhaps too slow to be of practical use. For the perturbation calculations presented in this report, however, such is not the case.

Figure 2 presents the CPU time required for initial derivation of successive orders of perturbation formulae in their crudest forms, while Figure 3 shows the time required to simplify these crude formulae into the compact forms listed in Appendices C-E. Combining the timing data from Figures 2 and 3, one sees that the amount of CPU time required to derive and simplify

\footnotetext{
${ }^{15}$ All Mathematica computations were performed on a Sun SPARC 5 workstation using Mathematica version 2.2 for Unix.

${ }^{16}$ Manipulating Mathematica output into a desired form is something of an art. The proper sequence of commands was found to be Simplify, which places all terms over a common denominator, followed by Expand, which separates this massive fraction into a sum of small terms and makes cancellations where appropriate.
} 
a given formula is in all cases on the order of $10^{\frac{1}{2} n}$ seconds. Furthermore, for a given value of $n$, CPU time scales linearly with $v$.

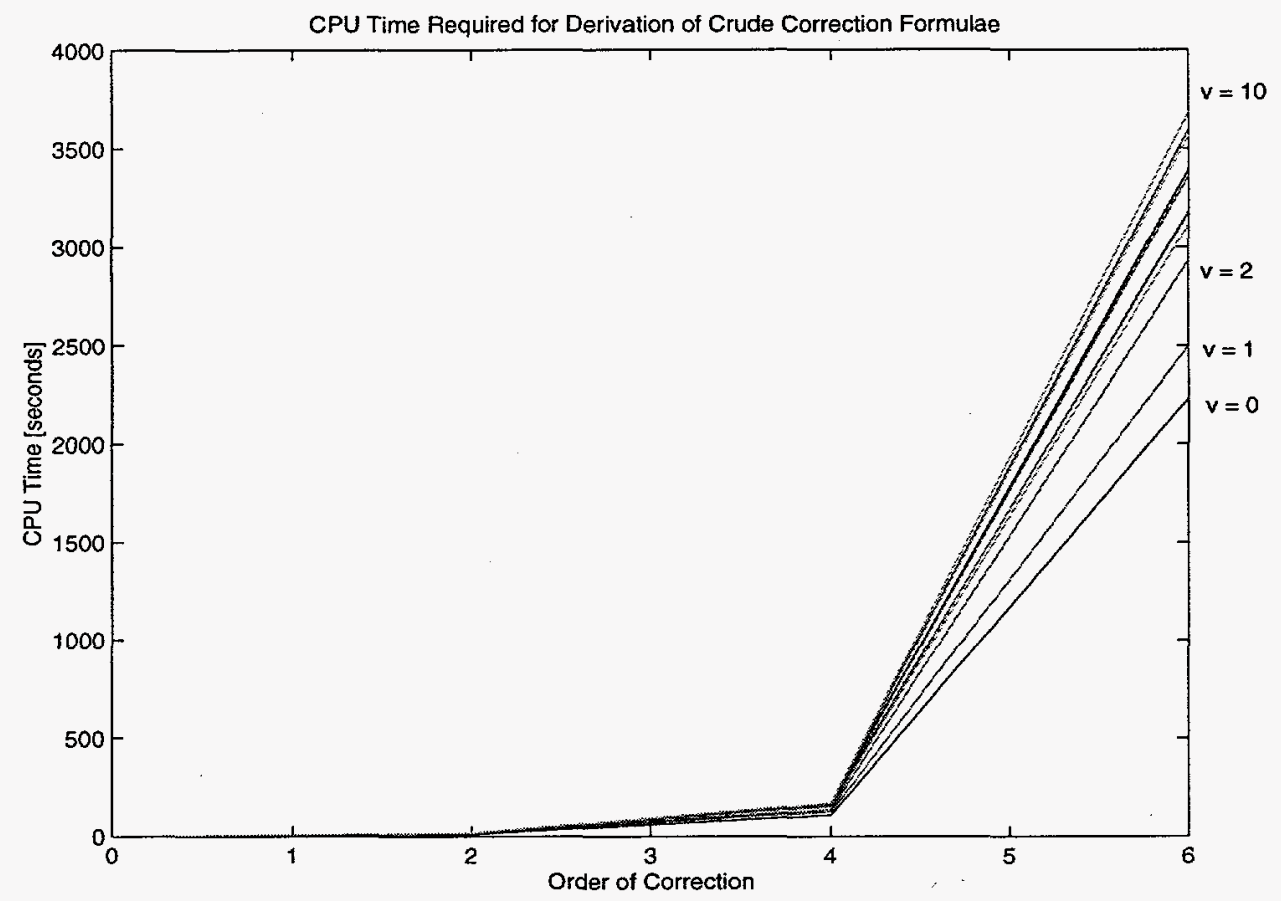

FIGURE 2. CPU time required by Mathematica to derive symbolic energy correction formulae. All computations were performed on a Sun SPARC 5 workstation using Mathematica version 2.2 for Unix. Results are shown for the first eleven vibrational states, ranging from $v=0$ to $v=10$.

Although CPU time does scale exponentially, two facts make this problem somewhat more tractable. First, because of the $v$ dependence of CPU time, the correction formulae for very low-lying vibrational states require significantly less time to derive and simplify than those for higher vibrational states (since negative values of $v$ are not allowed, summations over $v^{\prime} \neq v$ are considerably less involved for small $v$ ). Because Rayleigh-Schrödinger perturbation theory is applicable only to low-lying vibrational states, those formulae that are of primary interest are also those that require the least time to obtain.

Furthermore, it is worth noting that RSPERTURB is not the most efficient possible algorithm for deriving energy correction formulae because RSPERTURB recalculates the expansion coefficients $c_{v}^{(i)}$ from the general formula (58) each time they are needed. It would be enormously more efficient to first calculate as many Rayleigh-Schrödinger coefficients as are 
required, ${ }^{17}$ then store these expressions so that Mathematica may reference them during the course of a computation. In the case of diatomic molecules, however, the entire algorithm is wholly pedagogical in nature-the point is simply to demonstrate that arbitrary-order perturbation formulae can in fact be derived using computer algebra. In future work with polyatomic molecules (where emphasis shall be placed on obtaining actual numerical values for vibrational-rotational energies), a more efficient algorithm will be employed.

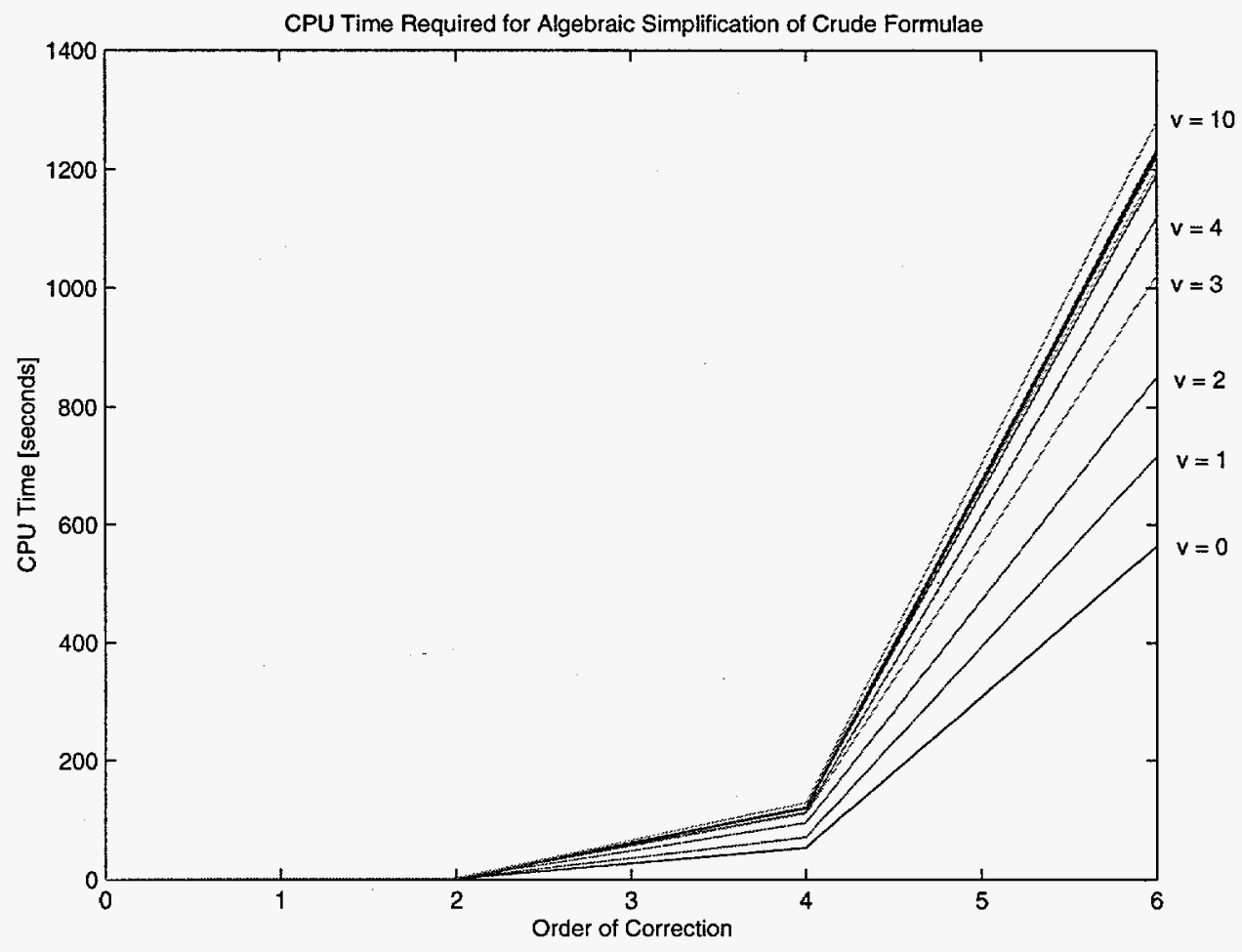

FIGURE 3. CPU time required by Mathematica to manipulate crude energy formulae into their simplest forms. Results are shown for the first eleven vibrational states.

\section{Numerical Results AND ANAlysis}

Numerical values for perturbation energies through the sixth order of correction for various molecules of interest are quickly obtained by using Equation (18) for the zeroth-order energy and the formulae listed in Appendices C-E for corrections to this energy. In a thorough $a b$ initio treatment, the force constants and equilibrium internuclear separation $R_{e}$ would be determined by means of electronic energy calculations [4, 12-14]. For ${ }^{1} \mathrm{H}_{2}$, this was accomplished

\footnotetext{
${ }^{17}$ To apply $n$ th-order perturbation theory, one needs all nonzero Rayleigh-Schrödinger coefficients $c_{v}^{(1)}, \ldots, c_{v}^{(\kappa)}$, where $n$ and $\kappa$ are related by Equation (66).
} 
by fitting existing $a b$ initio electronic energy data [33] to an eighth-degree Taylor polynomial; from Equations (6) and (22), each molecular force constant is just a coefficient of this polynomial divided by a factorial term. Notice from (27) that the ith perturbed Hamiltonian operator contains force constants through $k_{i+2}$, so the Taylor polynomial fit must be of at least eighth degree in order to apply sixth-order perturbation theory.

Using these Taylor force constants, vibrational-rotational energy levels for ${ }^{1} \mathrm{H}_{2}$ were calculated to the sixth order of Rayleigh-Schrödinger perturbation theory [RSPT(6)]. Because the Taylor polynomial approximation to the potential energy curve has some finite radius of convergence, the theoretical data obtained from this potential energy expansion become nonsensical beyond a certain value of $v$. (In this application it was found that beyond $v=4$ the calculated energy levels actually begin to decrease as the quantum numbers increase. Thus, theoretical calculations using this particular polynomial potential function cannot under any circumstances be extended beyond $v=4$.)

In Figure 4, RSPT(6) energies for each vibrational state $v=0$ through $v=4$ are plotted as a function of the rotational state $J$ and compared with experimental values [32]. The Taylor polynomial provides an excellent fit for $v=0$ and $v=1$; moreover, the difference between theoretical and experimental energies increases rapidly with $v$ but only slowly with $J$.

As noted in the preceding section, calculation of useful theoretical data concerning diatomic molecules is not the purpose of this report; rather, the objective of this work is to demonstrate that perturbation calculations can be carried out systematically to any order using computer algebra, as well as to explore the behavior of perturbation energy calculations for some test molecules. As such, only a few numerical results will be presented in order to verify that the energy correction formulae are indeed legitimate. Furthermore, force constants obtained from numerical fits of ab initio potential energy data will not be used, but instead an empirical function for the potential energy curve will be employed. The reason for this choice is that there exist $[2,34,45]$ several well-known analytic functions that can accurately represent potential energy curves for most diatomic molecules. Such functions cannot be employed in truly ab initio work, since their forms depend parametrically upon experimentally measured spectroscopic constants. However, for the purpose of testing the energy formulae of Appendices C-E, such accurate potential energy functions are extremely valuable and convenient.

The simplest (and, not inconsequentially, the most popular [34]) analytic function for the potential energy of a diatomic molecule is the Morse function [46]: 


$$
\mathrm{U}(Q)=D\left[1-e^{-\beta Q}\right]^{2}
$$

where $D$ is the molecular dissociation energy and $\beta$ is the so-called Morse parameter, whose value is given $[17,47]$ by

$$
\beta=\frac{\omega_{e}}{2 R_{e} \sqrt{B_{e} D}}
$$

Sprandel and Kern [8] have tabulated $D$ and $\beta$ for several diatomic molecules.

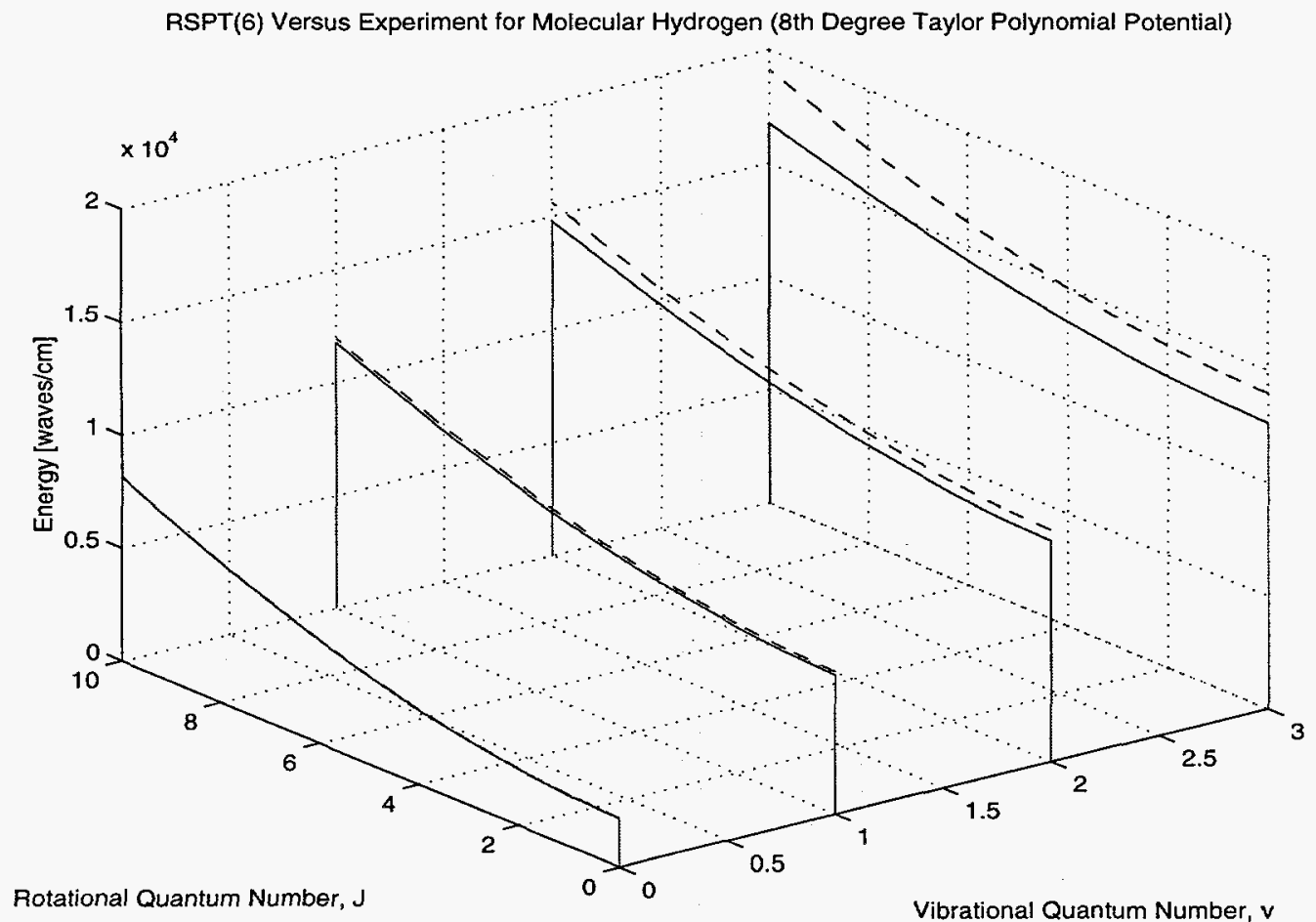

FIGURE 4. Energy calculations through sixth order for $X^{1} \Sigma^{+}{ }_{g}^{1} \mathrm{H}_{2}$, using force constants obtained from a Taylor polynomial fit of theoretical potential energy data [33]. Each solid line shows the theoretical energy of a particular vibrational state as a function of the rotational quantum number, and dashed lines represent corresponding experimental values [32].

Although Morse's function remains popular by virtue of its simplicity, the most accurate general-purpose empirical function for diatomic potentials is typically found $[34,45]$ to be the Hulburt-Hirschfelder function [47]. This function contains the parameters $D$ and $\beta$ from the Morse potential plus additional parameters $b$ and $c$ :

$$
\mathrm{U}(Q)=D\left[\left(1-e^{-\beta Q}\right)^{2}+c \beta^{3} Q^{3} e^{-2 \beta Q}(1+b \beta Q)\right] .
$$


Like $\beta$, the Hulburt-Hirschfelder parameters $b$ and $c$ can be written in terms of spectroscopic constants, but these formulae are quite complicated and have been omitted here. For the explicit forms of $b$ and $c$, as well as tabulated values of these parameters for many diatomic molecules, see Hulburt and Hirschfelder [47].

When an analytic potential energy function is used, symbolic expressions for the molecular force constants can be obtained by analytic differentiation of $\mathrm{U}$. Because $d R=d Q$ by (8), these derivatives may be taken either with respect to $R$ or with respect to $Q$, then evaluated at either $R=R_{e}$ or $Q=0$. For the Morse potential function (71), there exists [8] a simple closedform expression for these derivatives:

$$
k_{i}=\left\{\begin{array}{l}
2 \beta^{2} D, \text { if } i=e \\
(-\beta)^{i}\left(2^{i}-2\right) D, \text { if } i \geq 3
\end{array}\right\} .
$$

For the Hulburt-Hirschfelder potential function (73), no such closed-form expression exists; however, Mathematica can easily perform the requisite symbolic differentiation. For convenience, formulae for the Hulburt-Hirschfelder force constants (through $k_{10}$ ) obtained in this manner are listed in Appendix F.

For comparative purposes, RSPT(6) vibrational-rotational energies for ${ }^{1} \mathrm{H}_{2}$ were calculated by using first Morse and then Hulburt-Hirschfelder force constants. In Figures 5 and 6, RSPT(6) energies for each vibrational state $v=0$ through $v=10$ are plotted as functions of the rotational state $J$ and compared with experimental values [32]. For the lowest vibrational levels (i.e., $v \leq 4$ ), theoretical energies obtained from Hulburt-Hirschfelder force constants are essentially indistinguishable from experimental values. As $v$ increases, so too does the discrepancy between theory and experiment; this rift also increases (to a lesser extent) with increasing $J$. Similar behavior was observed in the Taylor polynomial potential energy curve (Figure 4), and is attributable to the fact that for low-lying states the rotational term in $\hat{\mathcal{H}}^{(i)}$ is much smaller than the vibrational term [27].

Although force constants from the Morse potential appear to provide a better fit for $v=7$ through $v=10$, the decision was made to use the Hulburt-Hirschfelder potential function for all calculations, since Rayleigh-Schrödinger perturbation theory is most applicable to the lowest vibrational levels. This last point cannot be overemphasized, and in performing such theoretical calculations it is imperative that one understand precisely how many vibrational and rotational energy levels can accurately be described using the given theory and all its intrinsic approximations and assumptions. 


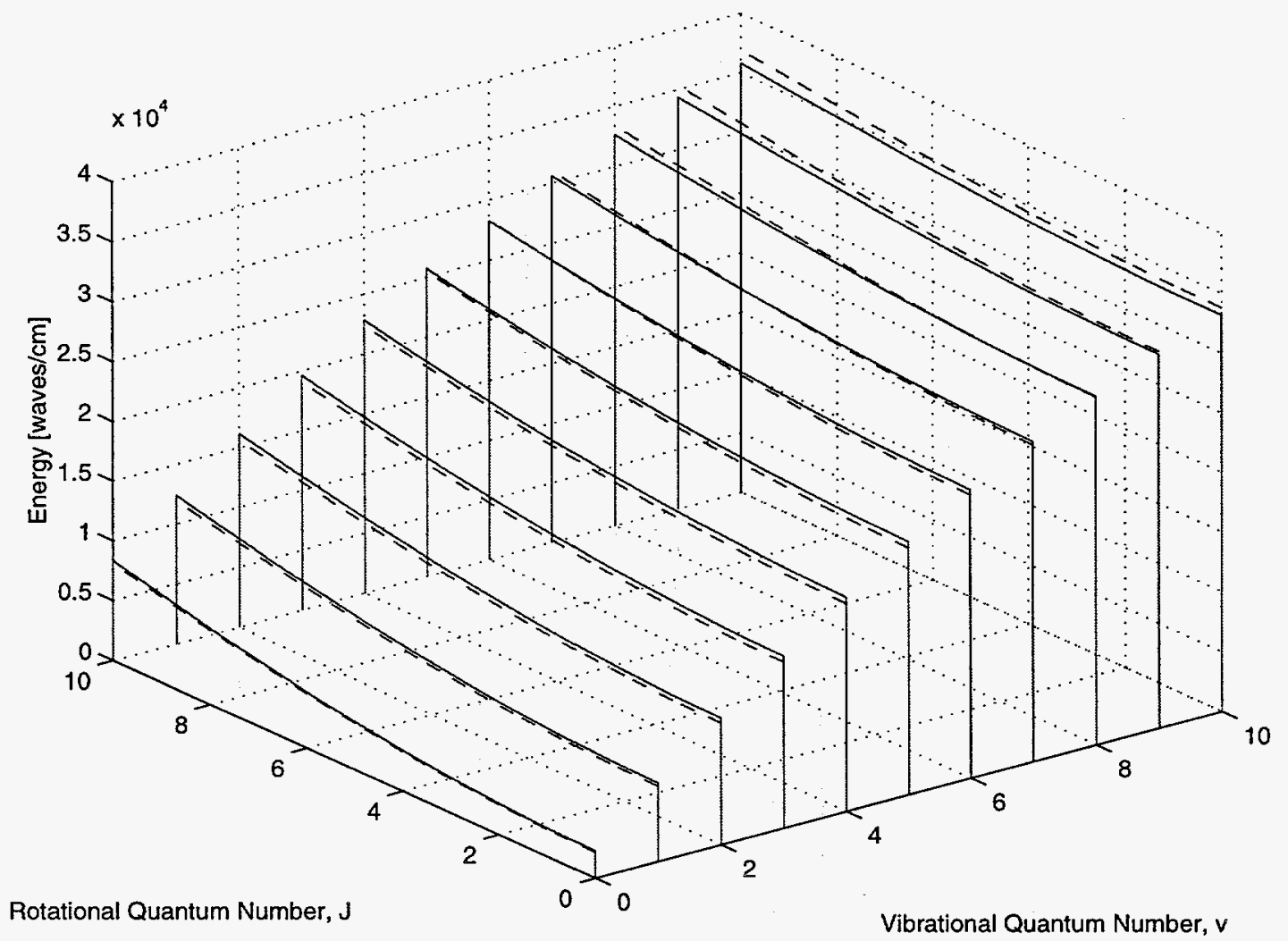

FIGURE 5. Energy calculations through sixth order for $X^{1} \Sigma^{+}{ }_{g}^{1} \mathrm{H}_{2}$, using force constants obtained from the Morse potential. Each solid line shows the theoretical energy for a particular vibrational state as a function of the rotational quantum number, and dashed lines represent experimental values [32]. Morse parameters were obtained from [8].

The maximum vibro-rotational energy that can be calculated to a given level of accuracy depends upon the potential energy surface, the order of perturbation theory, and the molecule itself. In what follows, a paradigmatic analysis of the accuracy of RSPT(6) calculations to ${ }^{1} \mathrm{H}_{2}$ is provided, beginning with a look at the sequence of energy corrections for this molecule.

Table 1 lists the individual correction terms for several different vibro-rotational states of ${ }^{1} \mathrm{H}_{2}$. Several important trends in perturbation energy corrections, which are true for nearly all diatomic molecules, are exemplified by this data. First, note that corrections to the zeroth-order energy are significantly smaller for $v=0$ than for $v=1$. This difference in the relative magnitudes of correction terms is even more pronounced at larger values of $v$ and is illustrative of a general trend: each order of perturbation correction becomes larger (in an absolute sense) as $v$ increases. This is not surprising, given that the harmonic oscillator model becomes 
increasingly less accurate for higher and higher vibrational energy levels; for highly excited vibrational states, significant correction to this idealized model is required.

RSPT(6) Versus Experiment for Molecular Hydrogen (Hulburt-Hirschfelder Potential)

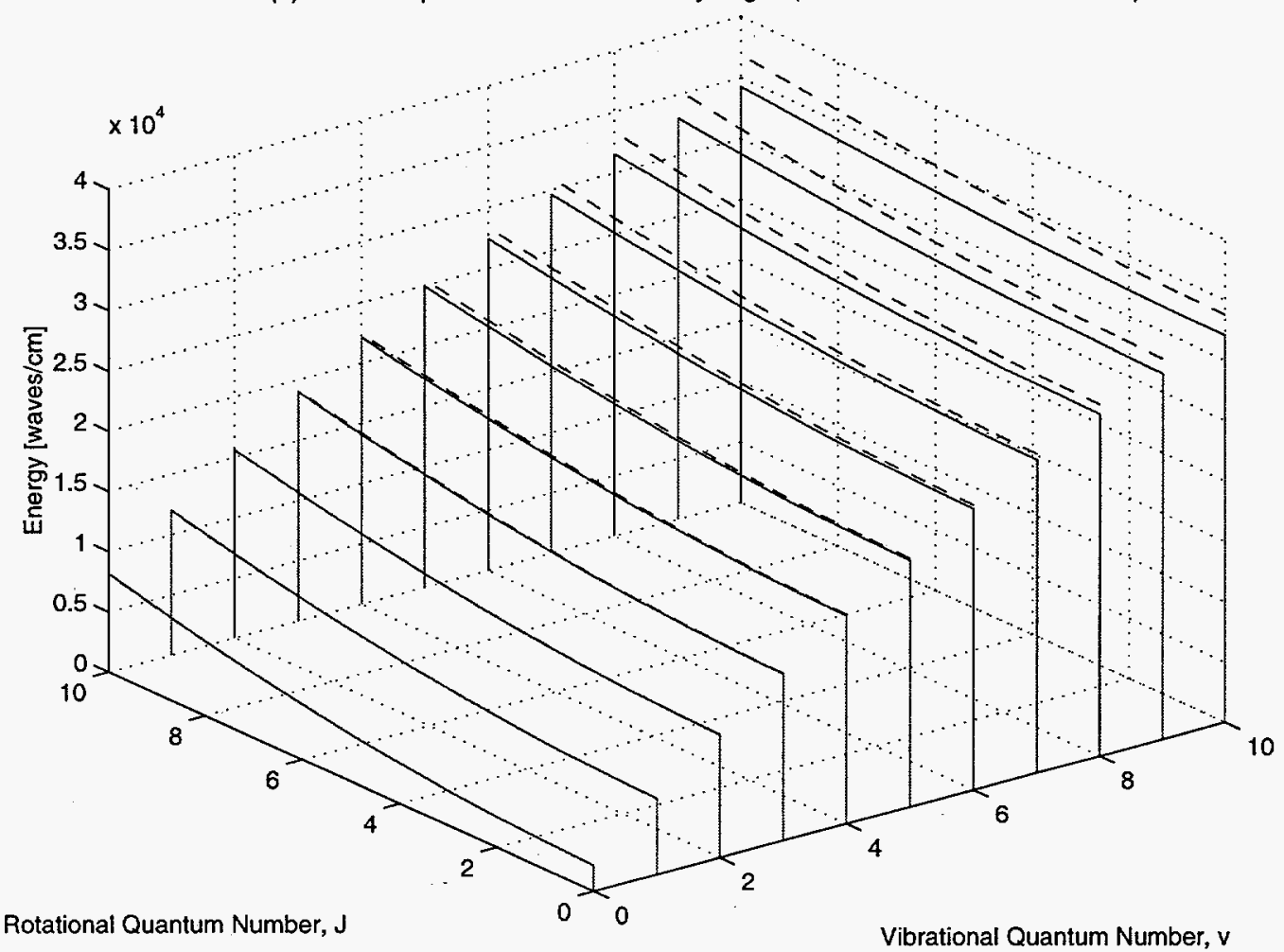

FIGURE 6. Energy calculations through sixth order for $X^{1} \Sigma^{+}{ }_{g}{ }^{1} \mathrm{H}_{2}$, using force constants obtained from the Hulburt-Hirschfelder potential. Each solid line shows the theoretical energy of a particular vibrational state as a function of the rotational quantum number, and dashed lines represent experimental values [32]. HulburtHirschfelder parameters were obtained from [47].

TABLE 1. Energy correction terms for six vibrational-rotational states of ${ }^{1} \mathrm{H}_{2}$. The tilde over the energy correction indicates division by Planck's constant times the speed of light in order to convert standard SI energy units into waves per centimeter (or wavenumbers), the standard units of molecular spectroscopy.

\begin{tabular}{|c|c|c|c|c|c|c|}
\hline $\begin{array}{l}\text { Correction } \\
\text { Term }\end{array}$ & \multicolumn{3}{|c|}{$\begin{array}{l}\text { Vibro-Rotational Energy Contributions, } \\
\qquad \widetilde{E}_{v, J}^{(i)}[\text { waves } / \mathrm{cm}]\end{array}$} & \multicolumn{3}{|c|}{$\begin{array}{l}\text { Vibro-Rotational Energy Contributions, } \\
\qquad \widetilde{E}_{v, J}^{(i)}[\text { waves } / \mathrm{cm}]\end{array}$} \\
\hline & $v=\mathbf{0}, \boldsymbol{J}=\mathbf{0}$ & $v=\mathbf{0}, \mathbf{J}=\mathbf{1}$ & $v=0, J=2$ & $v=1, \mathrm{~J}=0$ & $v=2, J=0$ & $v=3, J=0$ \\
\hline$E^{(0)}$ & 2202.42 & 2324.14 & 2567.58 & 6607.27 & 11012.12 & 15416.97 \\
\hline$E^{(2)}$ & -23.34 & -26.59 & -34.21 & -274.04 & -775.45 & -1527.56 \\
\hline$E^{(4)}$ & -0.52 & -0.49 & -0.42 & 1.99 & 45.91 & 101.48 \\
\hline$E^{(6)}$ & 0.02 & 0.02 & 0.02 & -0.51 & -5.05 & -20.57 \\
\hline
\end{tabular}


Table 1 also demonstrates, however, that energy increases relatively slowly with $J$. Although this is illustrated in Table 1 only for the ground vibrational state, it is in fact a general trend for low-lying vibrational and rotational states: within a given vibrational state, energy increases slowly with $J$, but within a given rotational state, energy increases quite rapidly with $v{ }^{18}$ This, in fact, is the trend illustrated in Figures 5 and 6, and is due to the fact [27] that the vibrational potential energy series (6) is term by term much larger than the rotational potential energy series (11).

TABLE 2. Complete results of RSPT(6) vibro-rotational energy calculations for $X^{1} \Sigma_{g}^{+}{ }^{1} \mathrm{H}_{2}$ (tabulated in waves per centimeter); the lower entry in each cell is an experimental value [32]. Theoretical calculations were performed using Hulburt-Hirschfelder force constants, where the Hulburt-Hirschfelder parameters were obtained from [47].

\begin{tabular}{|c|c|c|c|c|c|c|c|c|c|c|c|}
\hline & $J=\mathbf{0}$ & $J=1$ & $J=2$ & $J=3$ & $J=4$ & $J=5$ & $J=6$ & $J=7$ & $J=8$ & $J=9$ & $J=10$ \\
\hline$v=\mathbf{0}$ & $\begin{array}{l}2178.59 \\
2179.30\end{array}$ & $\begin{array}{l}2297.08 \\
2297.78\end{array}$ & $\begin{array}{l}2532.96 \\
2533.57\end{array}$ & $\begin{array}{l}2884.09 \\
2884.42\end{array}$ & $\begin{array}{l}3347.34 \\
3346.94\end{array}$ & $\begin{array}{l}3918.67 \\
3916.59\end{array}$ & $\begin{array}{l}4593.28 \\
4587.71\end{array}$ & $\begin{array}{l}5365.67 \\
5353.51\end{array}$ & $\begin{array}{l}6229.81 \\
6206.08\end{array}$ & $\begin{array}{l}7179.21 \\
7136.36\end{array}$ & $\begin{array}{l}8206.94 \\
8134.16\end{array}$ \\
\hline$v=1$ & $\begin{array}{l}6334.71 \\
6340.49\end{array}$ & $\begin{array}{l}6447.26 \\
6453.15\end{array}$ & $\begin{array}{l}6671.22 \\
6677.32\end{array}$ & $\begin{array}{l}7004.58 \\
7010.73\end{array}$ & $\begin{array}{l}7444.28 \\
7449.96\end{array}$ & $\begin{array}{l}7986.38 \\
7990.47\end{array}$ & $\begin{array}{l}8626.23 \\
8626.60\end{array}$ & $\begin{array}{l}9358.52 \\
9351.52\end{array}$ & $\begin{array}{l}10177.4 \\
10157.3\end{array}$ & $\begin{array}{l}11076.6 \\
11035.0\end{array}$ & $\begin{array}{l}12049.5 \\
11974.2\end{array}$ \\
\hline$v=2$ & $\begin{array}{l}10246.7 \\
10266.3\end{array}$ & $\begin{array}{l}10353.4 \\
10373.9\end{array}$ & $\begin{array}{l}10565.6 \\
10588.0\end{array}$ & $\begin{array}{l}10881.3 \\
10906.2\end{array}$ & $\begin{array}{l}11297.6 \\
11325.1\end{array}$ & $\begin{array}{l}11810.7 \\
11840.3\end{array}$ & $\begin{array}{l}12415.9 \\
12445.9\end{array}$ & $\begin{array}{l}13108.2 \\
13135.3\end{array}$ & $\begin{array}{l}13881.8 \\
13900.4\end{array}$ & $\begin{array}{l}14730.6 \\
14732.3\end{array}$ & $\begin{array}{l}15648.2 \\
15620.6\end{array}$ \\
\hline$v=3$ & $\begin{array}{l}13914.8 \\
13961.7\end{array}$ & $\begin{array}{l}14015.4 \\
14065.2\end{array}$ & $\begin{array}{l}14215.8 \\
14271.0\end{array}$ & $\begin{array}{l}14513.8 \\
14576.8\end{array}$ & $\begin{array}{l}14906.4 \\
14979.3\end{array}$ & $\begin{array}{l}15390.1 \\
15473.9\end{array}$ & $\begin{array}{l}15960.3 \\
16054.8\end{array}$ & $\begin{array}{l}16611.9 \\
16715.4\end{array}$ & $\begin{array}{l}17339.2 \\
17447.6\end{array}$ & $\begin{array}{l}18136.6 \\
18242.3\end{array}$ & $\begin{array}{l}18997.4 \\
19089.4\end{array}$ \\
\hline$v=4$ & $\begin{array}{l}17335.4 \\
17431.4\end{array}$ & $\begin{array}{l}17430.0 \\
17531.7\end{array}$ & $\begin{array}{l}17618.2 \\
17731.1\end{array}$ & $\begin{array}{l}17897.9 \\
18027.4\end{array}$ & $\begin{array}{l}18266.4 \\
18417.0\end{array}$ & $\begin{array}{l}18719.8 \\
18895.6\end{array}$ & $\begin{array}{l}19253.8 \\
19457.3\end{array}$ & $\begin{array}{l}19863.2 \\
20095.5\end{array}$ & $\begin{array}{l}20542.7 \\
20802.1\end{array}$ & $\begin{array}{l}21286.4 \\
21568.0\end{array}$ & $\begin{array}{l}22087.9 \\
22383.2\end{array}$ \\
\hline$v=5$ & $\begin{array}{l}20501.9 \\
20680.4\end{array}$ & $\begin{array}{l}20590.1 \\
20778.1\end{array}$ & $\begin{array}{l}20765.6 \\
20972.4\end{array}$ & $\begin{array}{l}21026.3 \\
21260.9\end{array}$ & $\begin{array}{l}21369.4 \\
21640.2\end{array}$ & $\begin{array}{l}21791.2 \\
22105.9\end{array}$ & $\begin{array}{l}22287.2 \\
22652.2\end{array}$ & $\begin{array}{l}22852.5 \\
23272.4\end{array}$ & $\begin{array}{l}23481.5 \\
23958.5\end{array}$ & $\begin{array}{l}24168.4 \\
24701.5\end{array}$ & $\begin{array}{l}24907.0 \\
25491.1\end{array}$ \\
\hline$v=6$ & $\begin{array}{l}23403.8 \\
23713.6\end{array}$ & $\begin{array}{l}23485.3 \\
23808.8\end{array}$ & $\begin{array}{l}23647.4 \\
23998.0\end{array}$ & $\begin{array}{l}23888.0 \\
24279.1\end{array}$ & $\begin{array}{l}24204.3 \\
24648.5\end{array}$ & $\begin{array}{l}24592.5 \\
25101.8\end{array}$ & $\begin{array}{l}25048.2 \\
25633.3\end{array}$ & $\begin{array}{l}25566.3 \\
26236.2\end{array}$ & $\begin{array}{l}26141.4 \\
26902.7\end{array}$ & $\begin{array}{l}26767.7 \\
27623.5\end{array}$ & $\begin{array}{l}27438.7 \\
28388.7\end{array}$ \\
\hline$v=7$ & $\begin{array}{l}26027.3 \\
26535.8\end{array}$ & $\begin{array}{l}26101.7 \\
26627.8\end{array}$ & $\begin{array}{l}26249.4 \\
26810.7\end{array}$ & $\begin{array}{l}26468.5 \\
27082.2\end{array}$ & $\begin{array}{l}26756.0 \\
27439.0\end{array}$ & $\begin{array}{l}27108.2 \\
27876.5\end{array}$ & $\begin{array}{l}27520.5 \\
28389.1\end{array}$ & $\begin{array}{l}27988.0 \\
28970.0\end{array}$ & $\begin{array}{l}28505.0 \\
29611.4\end{array}$ & $\begin{array}{l}29065.5 \\
30304.2\end{array}$ & $\begin{array}{l}29663.3 \\
31381.2\end{array}$ \\
\hline$v=8$ & $\begin{array}{l}28355.2 \\
29151.9\end{array}$ & $\begin{array}{l}28421.9 \\
29239.0\end{array}$ & $\begin{array}{l}28554.1 \\
29412.3\end{array}$ & $\begin{array}{l}28749.9 \\
29669.0\end{array}$ & $\begin{array}{l}29006.3 \\
30006.3\end{array}$ & $\begin{array}{l}29319.5 \\
30419.5\end{array}$ & $\begin{array}{l}29684.8 \\
30903.0\end{array}$ & $\begin{array}{l}30097.2 \\
31450.1\end{array}$ & $\begin{array}{l}30551.0 \\
32052.9\end{array}$ & $\begin{array}{l}31040.0 \\
32702.3\end{array}$ & $\begin{array}{l}31557.7 \\
33388.3\end{array}$ \\
\hline$v=9$ & $\begin{array}{l}30366.7 \\
31566.8\end{array}$ & $\begin{array}{l}30424.9 \\
31646.1\end{array}$ & $\begin{array}{l}30540.3 \\
31803.4\end{array}$ & $\begin{array}{l}30710.8 \\
32036.7\end{array}$ & $\begin{array}{l}30933.3 \\
32342.7\end{array}$ & $\begin{array}{l}31204.0 \\
32716.7\end{array}$ & $\begin{array}{l}31518.1 \\
33153.4\end{array}$ & $\begin{array}{l}31870.4 \\
33645.9\end{array}$ & $\begin{array}{l}32254.9 \\
34186.4\end{array}$ & $\begin{array}{l}32665.5 \\
34765.8\end{array}$ & $\begin{array}{l}33095.4 \\
35374.2\end{array}$ \\
\hline$=10$ & $\begin{array}{l}32037.5 \\
33785.4\end{array}$ & $\begin{array}{l}32086.5 \\
33852.3\end{array}$ & $\begin{array}{l}32183.5 \\
33984.9\end{array}$ & $\begin{array}{l}32326.3 \\
34181.2\end{array}$ & $\begin{array}{l}32511.8 \\
34437.9\end{array}$ & $\begin{array}{l}32735.9 \\
34750.4\end{array}$ & $\begin{array}{l}32993.8 \\
35113.4\end{array}$ & $\begin{array}{l}33280.1 \\
35519.9\end{array}$ & $\begin{array}{l}33588.7 \\
35962.4\end{array}$ & $\begin{array}{l}33913.1 \\
36431.7\end{array}$ & $\begin{array}{l}34246.3 \\
36917.8\end{array}$ \\
\hline
\end{tabular}

\footnotetext{
${ }^{18}$ For highly excited vibrational and rotational states, this simple qualitative model breaks down, since vibrational and rotational level spacings exhibit opposite trends: vibrational level spacing decreases as $v$ increases, while rotational level spacing increases as $J$ increases $[17,34]$. However, for the low-lying vibrational-rotational states to which Rayleigh-Schrödinger perturbation theory is applicable, the vibrational corrections will indeed be much larger than the rotational ones.
} 
Table 2 shows the complete results of RSPT(6) calculations for ${ }^{1} \mathrm{H}_{2}$, along with experimental vibro-rotational energies obtained from spectroscopic data [32]; for convenience, the relative differences between theoretical and experimental energies are tabulated in Table 3. The data in Table 3 indicate excellent agreement between theory and experiment for low-lying vibrational states (for instance, when $v \leq 3$ the difference between theoretical and experimental energies is less than one percent for all eleven rotational levels considered); moreover, the relative differences in Table 3 are not altogether large even for higher vibrational states. However, it should be noted that when $v>3$ the absolute difference between theoretical and experimental energies is often on the order of several hundred wavenumbers. Thus, it appears that for $v>3$ one might wish to resort to eighth- or higher-order perturbation corrections.

Much of the discrepancy between theoretical and experimental energies in Table 2 is obviously due to the fact that Rayleigh-Schrödinger perturbation theory works well only near the equilibrium geometry; as $v$ and $J$ increase, the two nuclei of the diatomic molecule spend more and more of their time separated by large values of $R$ and hence away from the minimum-energy configuration. ${ }^{19}$ However, some portion of the discrepancy in Table 2 can be attributed to the experimental values themselves.

TABLE 3. Relative (percent) difference between the theoretical and experimental energies in Table 2. Negative values indicate theoretical energies that lie below experimental ones.

\begin{tabular}{|c|c|c|c|c|c|c|c|c|c|c|c|}
\hline & $J=0$ & $J=1$ & $J=2$ & $J=3$ & $J=4$ & $J=5$ & $J=6$ & $J=7$ & $J=8$ & $J=9$ & $J=10$ \\
\hline$v=0$ & -0.03 & -0.03 & -0.02 & -0.01 & 0.01 & 0.05 & 0.12 & 0.23 & 0.38 & 0.60 & 0.89 \\
\hline$v=1$ & -0.09 & -0.09 & -0.09 & -0.09 & -0.08 & -0.05 & 0.00 & 0.07 & 0.20 & 0.38 & 0.63 \\
\hline$v=2$ & -0.19 & -0.20 & -0.21 & -0.23 & -0.24 & -0.25 & -0.24 & -0.21 & -0.13 & -0.01 & 0.18 \\
\hline$v=3$ & -0.34 & -0.35 & -0.39 & -0.43 & -0.49 & -0.54 & -0.59 & -0.62 & -0.62 & -0.58 & -0.48 \\
\hline$v=4$ & -0.55 & -0.58 & -0.64 & -0.72 & -0.82 & -0.93 & -1.05 & -1.16 & -1.25 & -1.31 & -1.32 \\
\hline$v=5$ & -0.86 & -0.90 & -0.99 & -1.10 & -1.25 & -1.42 & -1.61 & -1.80 & -1.99 & -2.16 & -2.29 \\
\hline$v=6$ & -1.31 & -1.36 & -1.46 & -1.61 & -1.80 & -2.03 & -2.28 & -2.55 & -2.83 & -3.10 & -3.35 \\
\hline$v=7$ & -1.92 & -1.98 & -2.09 & -2.27 & -2.49 & -2.76 & -3.06 & -3.39 & -3.74 & -4.09 & -5.47 \\
\hline$v=8$ & -2.73 & -2.79 & -2.92 & -3.10 & -3.33 & -3.62 & -3.94 & -4.30 & -4.69 & -5.08 & -5.48 \\
\hline$v=9$ & -3.80 & -3.86 & -3.97 & -4.14 & -4.36 & -4.62 & -4.93 & -5.28 & -5.65 & -6.04 & -6.44 \\
\hline$v=10$ & -5.17 & -5.22 & -5.30 & -5.43 & -5.59 & -5.80 & -6.04 & -6.31 & -6.60 & -6.91 & -7.24 \\
\hline
\end{tabular}

\footnotetext{
${ }^{19}$ It is easy to visualize how an increase in vibrational energy stretches a diatomic molecule away from its equilibrium geometry. However, an increase in rotational energy has the same effect due to centrifugal stretching, as discussed in Section IV.
} 
Virtually all tabulated experimental vibro-rotational energy data (including those listed in Table 2) come from energy formulae that are power series in $\left(v+\frac{1}{2}\right)$ and $J(J+1)$, and whose coefficients are the important spectroscopic constants. These formulae have the form

$$
\tilde{E}_{v, J}=\tilde{\mathrm{A}}_{v}\left(v+\frac{1}{2}\right)+\tilde{\mathrm{B}}_{v} J(J+1)+\tilde{\mathrm{D}}_{v} J^{2}(J+1)^{2}+\tilde{\mathrm{Y}}_{00},
$$

where tildes indicate units of waves per centimeter. While $\tilde{\mathrm{Y}}_{00}$ is simply a constant with no dependence on $v$ or $J$, each of $\widetilde{\mathrm{A}}_{v}, \widetilde{\mathrm{B}}_{v}$, and $\widetilde{\mathrm{D}}_{v}$ is a power series ${ }^{20}$ in $\left(v+\frac{1}{2}\right)$ :

$$
\begin{aligned}
& \tilde{\mathrm{A}}_{v}=\tilde{\omega}_{e}+\tilde{\omega}_{e} x_{e}\left(v+\frac{1}{2}\right)+\tilde{\omega}_{e} y_{e}\left(v+\frac{1}{2}\right)^{2}+\cdots, \\
& \widetilde{\mathrm{B}}_{v}=\widetilde{B}_{e}+\tilde{\alpha}_{e}\left(v+\frac{1}{2}\right)+\tilde{\beta}_{e}\left(v+\frac{1}{2}\right)^{2}+\tilde{\gamma}_{e}\left(v+\frac{1}{2}\right)^{3}+\cdots, \\
& \tilde{\mathrm{D}}_{v}=\tilde{D}_{e}+\tilde{\delta}_{e}\left(v+\frac{1}{2}\right)+\tilde{\varepsilon}_{e}\left(v+\frac{1}{2}\right)^{2}+\cdots
\end{aligned}
$$

$\widetilde{A}_{v}$ is an anharmonicity series whose first terms corresponds to $\omega_{e}$ from (18) and whose remaining constant coefficients $\tilde{\omega}_{e} x_{e}, \tilde{\omega}_{e} y_{e}$, etc., are known as anharmonicity constants. These terms correct for the non-parabolic nature of the potential energy curve and tend to decrease vibrational level spacing as $v$ increases [17]. The series $\tilde{\mathrm{B}}_{v}$ (whose constant coefficients $\tilde{\alpha}_{e}, \tilde{\beta}_{e}$, etc., are known as coupling constants) defines an effective rotational constant for vibrational level $v$ that takes into account vibrational-rotational coupling; the first term in this series corresponds to $B_{e}$ in (18). Finally, the centrifugal distortion series $\tilde{\mathrm{D}}_{v}$ (whose coefficients are the so-called centrifugal distortion constants) accounts for centrifugal stretching effects [48].

Truncated versions of the power series (76-78) are obtained from numerical fits of experimental vibro-rotational energy data; the number of terms that are included in the final experimental energy equation (75) depends upon the accuracy of available experimental data and therefore varies from molecule to molecule. It must be stressed, however, that the experimental energy formula (75) is an approximation [48] and is not valid for all values of $v$ and $J$. In the case of the ${ }^{1} \mathrm{H}_{2}$ data in Table 2, the fit is valid only for $v \leq 3$ [32], which is one of the worst experimental energy fits of any listed in [32]. (Dunham [41] predicts that this should, in fact, be the worst fit, since ${ }^{1} \mathrm{H}_{2}$ is the lightest of all molecules). Not coincidentally, the relative differences (Table 3) between experimental and theoretical energies are largest when $v>3$.

\footnotetext{
${ }^{20}$ Most texts on experimental spectroscopy include explicit negative signs for $\tilde{\omega}_{e} x_{e}, \tilde{\alpha}_{e}$, and $\tilde{D}_{e}$; however, in deference to mathematical generality the forms in (76-78) will be used in this report, and appropriate signs will be incorporated into numerical values of spectroscopic constants.
} 
Because of this breakdown in the Dunham energy series approximation, the theoretical energies for $v>3$ in Table 2 may, in fact, be more accurate than existing experimental values might lead one to believe.

The small relative differences in Table 3 can also be somewhat misleading, for it is known that the Taylor series (6) and (11) fail to converge for all values of $v$ and $J$ listed in this table. An estimate of the maximum values of $v$ and $J$ for which convergence is guaranteed can be obtained by examining the radii of convergence of the potential energy series (6) and (11). In Section III it was easily shown that series (11) converges whenever $Q<R_{e}$. The radius of convergence for (6) is known [9] to be approximately the same, although this radius is difficult to determine exactly.

Figure 7 depicts a plot of the Hulburt-Hirschfelder potential energy curve for ${ }^{1} \mathrm{H}_{2}$ obtained by using the Hulburt-Hirschfelder parameters listed in [47]; overlaid onto this plot are the RSPT(6) vibrational energy levels for the ground rotational state and the $J=10$ rotational state. The vertical line in Figure 7 is located at the radius of convergence, $Q=R_{e}$. Although the quantum-mechanical harmonic oscillator may tunnel out of the potential well of Figure 7, the wave function falls off very rapidly for values of $Q$ outside this well $[17,27]$. Hence, to a good approximation one may restrict the normal coordinate to values within the potential energy well.

Note that for vibrational levels above $v=5$ (in the ground rotational state) and above $v=3$ (in the $J=10$ rotational state), the normal coordinate may drift beyond $R_{e}$ yet still be within the potential well. For these energy levels, the perturbation series (32) and (33) cannot be assumed to converge for all values of $Q$, so the perturbation treatment presented herein is not applicable. Since $J=10$ and $J=0$ are, respectively, the highest and lowest rotational levels examined for ${ }^{1} \mathrm{H}_{2}$, Figure 7 establishes boundary conditions for convergence of the perturbation series for this molecule. For the rotational levels $0 \leq J \leq 10$, one anticipates convergent perturbation series up to at least the $v=3$ vibrational level but no higher than the $v=5$ vibrational level.

One last comment concerning the accuracy of molecular hydrogen calculations is in order. This molecule (and, in particular, the diprotium isotope examined here) is something of a worst-case scenario. Because it is the lightest molecule, high-order energy corrections for $\mathrm{H}_{2}$ should be the most significant of any diatomic molecule [41]. Furthermore, the breakdown of the Born-Oppenheimer approximation is known [49] to be more significant for isotopomers of $\mathrm{H}_{2}$ 
than for other diatomic molecules. Thus, $\mathrm{H}_{2}$ represents something of a lower limit to the accuracy of perturbation calculations.

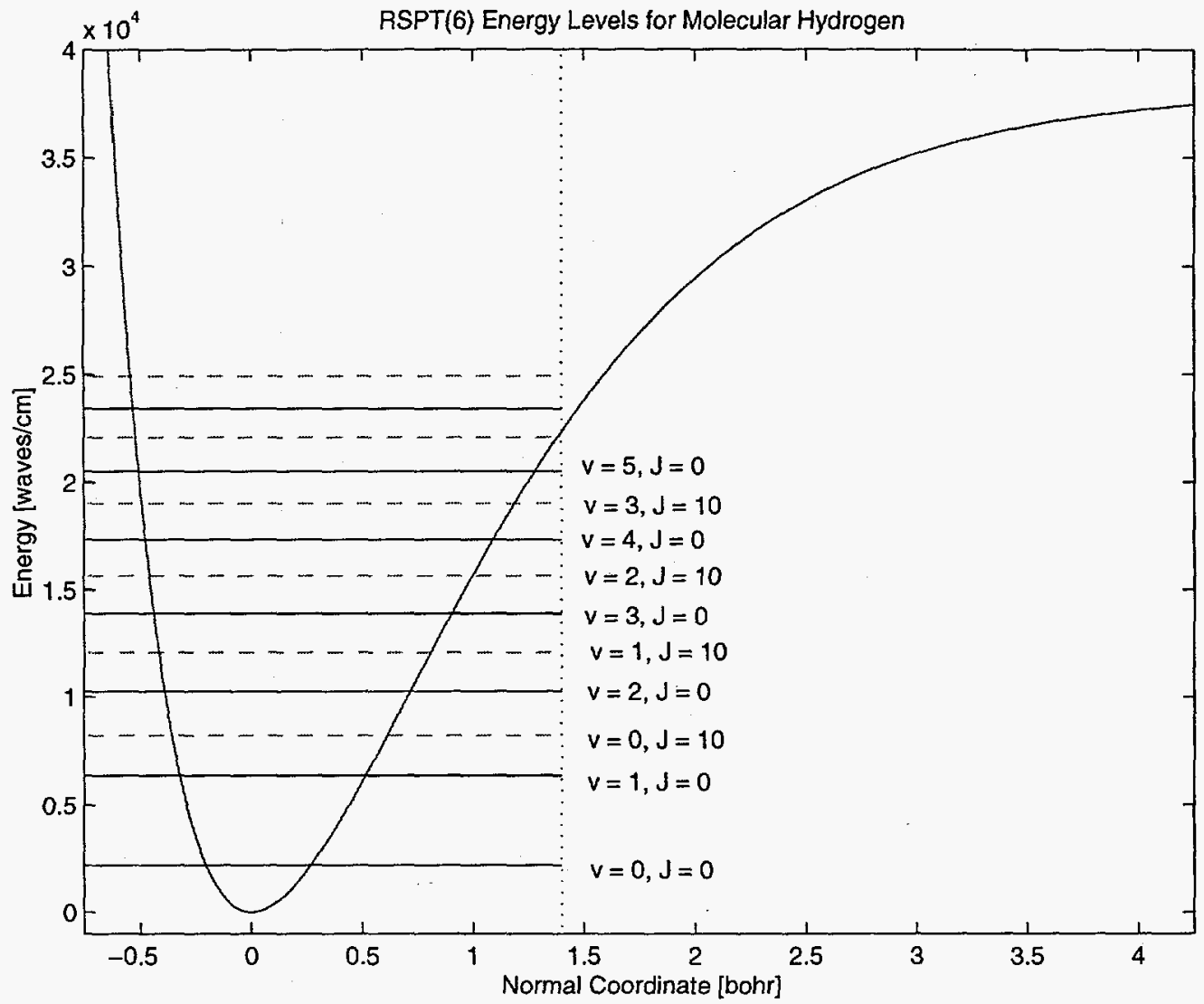

FIGURE 7. Hulburt-Hirschfelder potential energy curve and vibro-rotational energy levels for the ground rotational state (solid lines) and the $J=10$ rotational state (dashed lines) of $X^{1} \Sigma_{g}^{+}{ }^{1} \mathrm{H}_{2}$. These energies were obtained from RSPT(6) calculations by using Hulburt-Hirschfelder force constants, where the Hulburt-Hirschfelder parameters $b$ and $c$ were taken from [47]. The dotted vertical line is located at $Q=R_{e}$.

TABLE 4. Experimental and RSPT(6) vibrational-rotational energies for $X^{1} \Sigma^{+}{ }_{g}^{14} \mathrm{~N}_{2}$ in units of waves per centimeter. Experimental values were taken from [32], while theoretical calculations used the Hulburt-Hirschfelder potential model with Hulburt-Hirschfelder parameters obtained from [47].

\begin{tabular}{|c|c|c|c|c|c|c|c|c|c|c|}
\hline$\tilde{E}_{v, J}$ & \multicolumn{2}{|c|}{$J=\mathbf{0}$} & \multicolumn{2}{c|}{$J=\mathbf{1}$} & \multicolumn{2}{c|}{$J=\mathbf{2}$} & \multicolumn{2}{c|}{$J=\mathbf{3}$} \\
\hline${ }^{14} \mathbf{N}_{2}$ & RSPT(6) & Exp. & RSPT(6) & Exp. & RSPT(6) & Exp. & RSPT(6) & Exp. & RSPT(6) & Exp. \\
\hline$v=\mathbf{0}$ & 1176.69 & 1175.70 & 1180.69 & 1179.68 & 1188.68 & 1187.64 & 1200.68 & 1199.58 & 1216.67 & 1215.49 \\
\hline$v=\mathbf{1}$ & 3507.55 & 3505.62 & 3511.51 & 3509.56 & 3519.43 & 3517.45 & 3531.32 & 3529.28 & 3547.16 & 3545.06 \\
\hline$v=\mathbf{2}$ & 5808.20 & 5806.89 & 5812.12 & 5810.77 & 5819.97 & 5818.58 & 5831.74 & 5830.31 & 5847.43 & 5845.95 \\
\hline$v=\mathbf{3}$ & 8077.78 & 8079.39 & 8081.65 & 8083.27 & 8089.42 & 8091.02 & 8101.08 & 8102.64 & 8116.62 & 8118.14 \\
\hline$v=\mathbf{4}$ & 10315.4 & 10323.2 & 10319.2 & 10327.0 & 10326.9 & 10334.7 & 10338.5 & 10334.7 & 10353.8 & 10361.6 \\
\hline
\end{tabular}


Tables 4 through 7 present experimental and RSPT(6) energies for several other representative diatomic molecules, while Tables 8 and 9 list the relative differences between theory and experiment for each set of calculations. Notice that this difference is much smaller for heavier molecules $\left({ }^{14} \mathrm{~N}_{2}\right.$ and $\left.{ }^{12} \mathrm{C}^{16} \mathrm{O}\right)$ than for lighter ones $\left({ }^{1} \mathrm{H}^{19} \mathrm{~F}\right.$ and $\left.{ }^{1} \mathrm{H}^{2} \mathrm{H}\right)$. This behavior affirms Dunham's result [41] for dinuclear vibrational-rotational energies: the accuracy of loworder perturbation calculations increases with the molecular reduced mass.

TABLE 5. Experimental and RSPT(6) vibrational-rotational energies for $X^{1} \Sigma^{+12} \mathrm{C}^{16} \mathrm{O}$ in units of waves per centimeter. Experimental values were taken from [32], while theoretical calculations used the Hulburt-Hirschfelder potential model with Hulburt-Hirschfelder parameters obtained from [47].

\begin{tabular}{|c|c|c|c|c|c|c|c|c|c|c|}
\hline$\widetilde{E}_{v, J}$ & \multicolumn{2}{|c|}{$J=\mathbf{0}$} & \multicolumn{2}{c|}{$J=\mathbf{1}$} & \multicolumn{2}{c|}{$J=\mathbf{2}$} & \multicolumn{2}{c|}{$J=\mathbf{3}$} & $J=4$ \\
\hline${ }^{12} \mathrm{C}^{16} \mathrm{O}$ & RSPT(6) & Exp. & RSPT(6) & Exp. & RSPT(6) & Exp. & RSPT(6) & Exp. & RSPT(6) & Exp. \\
\hline$v=\mathbf{0}$ & 1081.07 & 1081.59 & 1084.92 & 1085.31 & 1092.60 & 1093.12 & 1104.14 & 1104.66 & 1119.51 & 1120.03 \\
\hline$v=\mathbf{1}$ & 3223.08 & 3224.86 & 3226.89 & 3228.67 & 3234.51 & 3236.29 & 3245.94 & 3247.72 & 3261.17 & 3262.96 \\
\hline$v=\mathbf{2}$ & 5338.70 & 5341.65 & 5342.47 & 5345.42 & 5350.02 & 5352.97 & 5361.34 & 5364.30 & 5376.44 & 5379.40 \\
\hline$v=\mathbf{3}$ & 7427.71 & 7432.03 & 7431.44 & 7435.77 & 7438.92 & 7443.25 & 7450.14 & 7454.46 & 7465.09 & 7469.42 \\
\hline$v=\mathbf{4}$ & 9489.88 & 9496.06 & 9493.59 & 9499.76 & 9500.99 & 9507.17 & 9512.10 & 9518.28 & 9526.91 & 9533.10 \\
\hline
\end{tabular}

TABLE 6. Experimental and RSPT(6) vibrational-rotational energies for $X^{1} \Sigma^{+1} \mathrm{H}^{19} \mathrm{~F}$ in units of waves per centimeter. Experimental values were taken from [32], while theoretical calculations used the Hulburt-Hirschfelder potential model with Hulburt-Hirschfelder parameters obtained from [47].

\begin{tabular}{|c|c|c|c|c|c|c|c|c|c|c|}
\hline$\tilde{E}_{v, J}$ & \multicolumn{2}{|c|}{$J=\mathbf{0}$} & \multicolumn{2}{c|}{$J=\mathbf{1}$} & \multicolumn{2}{c|}{$J=\mathbf{2}$} & \multicolumn{2}{c|}{$J=\mathbf{3}=\mathbf{4}$} \\
\hline${ }^{1} \mathrm{H}^{19} \mathrm{~F}$ & RSPT(6) & Exp. & RSPT(6) & Exp. & RSPT(6) & Exp. & RSPT(6) & Exp. & RSPT(6) & Exp. \\
\hline$v=\mathbf{0}$ & 2054.70 & 2046.80 & 2095.73 & 2087.94 & 2177.79 & 2170.34 & 2300.75 & 2294.2 & 2464.47 & 2460.02 \\
\hline$v=\mathbf{1}$ & 6018.60 & 6008.23 & 6057.99 & 6048.05 & 6136.73 & 6129.21 & 6254.72 & 6254.73 & 6411.81 & 6429.14 \\
\hline$v=\mathbf{2}$ & 9813.65 & 9797.62 & 9851.46 & 9836.40 & 9927.04 & 9918.26 & 10040.3 & 10051.8 & 10191.1 & 10249.9 \\
\hline$v=\mathbf{3}$ & 13445.2 & 1341.97 & 13481.5 & 13457.7 & 13554.0 & 13542.2 & 13662.7 & 13690.1 & 13807.5 & 13926.8 \\
\hline$v=\mathbf{4}$ & 16916.0 & 16878.6 & 16950.8 & 16916.1 & 17020.3 & 17005.2 & 17124.6 & $\mathbf{1 7 1 7 3 . 8}$ & 17263.4 & $\mathbf{1 7 4 6 4 . 2}$ \\
\hline
\end{tabular}

The RSPT(6) calculations in Tables 4-7 are all based upon the Hulburt-Hirschfelder force constants listed in Appendix F. The values of $b$ and $c$ used in these equations were taken from those explicitly tabulated in [47], while the spectroscopic constants necessary to obtain $\beta$ were taken from more recent experimental data [32]. The spectroscopic constants listed in [32], however, differ from the ones that Hulburt and Hirschfelder [47] used to calculate $b$ and $c$, so these Hulburt-Hirschfelder parameters were also calculated from the spectroscopic constants listed in [32] (using the formulae in [47]). When force constants obtained in this manner were 
utilized in RSPT(6) calculations, the theoretical energies obtained for ${ }^{1} \mathrm{H}^{19} \mathrm{~F}$ and ${ }^{1} \mathrm{H}^{2} \mathrm{H}$ fell several wavenumbers closer to experimental values, thus demonstrating the importance of possessing an accurate potential energy function.

TABLE 7. Experimental and RSPT(6) vibrational-rotational energies for $X^{1} \Sigma^{+}{ }_{g}^{1} \mathrm{H}^{2} \mathrm{H}$ in units of waves per centimeter. Experimental values were taken from [32], while theoretical calculations used the Hulburt-Hirschfelder potential model with Hulburt-Hirschfelder parameters obtained from [47].

\begin{tabular}{|c|c|c|c|c|c|c|c|c|c|c|}
\hline$\widetilde{E}_{v, \boldsymbol{J}}$ & \multicolumn{2}{|c|}{$J=\mathbf{0}$} & \multicolumn{2}{c|}{$J=\mathbf{1}$} & \multicolumn{2}{c|}{$J=\mathbf{2}$} & \multicolumn{2}{c|}{$J=\mathbf{3}$} & \multicolumn{2}{c|}{$J=4$} \\
\hline${ }^{1} H^{2} H$ & RSPT(6) & Exp. & RSPT(6) & Exp. & RSPT(6) & Exp. & RSPT(6) & Exp. & RSPT(6) & Exp. \\
\hline$v=\mathbf{0}$ & 1890.33 & 1883.75 & 1979.58 & 1972.96 & 2157.47 & 2150.74 & 2422.78 & 2415.85 & 2773.71 & 2766.42 \\
\hline$v=\mathbf{1}$ & 5519.06 & 5515.90 & 5604.42 & 5601.02 & 5774.54 & 5770.64 & 6028.23 & 6023.50 & 6363.74 & 6357.73 \\
\hline$v=\mathbf{2}$ & 8962.80 & 8971.00 & 9044.32 & 9051.95 & 9206.76 & 9213.23 & 9448.96 & 9453.58 & 9769.20 & 9771.13 \\
\hline$v=\mathbf{3}$ & 12222.6 & 12253.2 & 12300.2 & 12329.9 & 12455.0 & 12482.7 & 12685.7 & 1271.04 & 12990.6 & 13011.0 \\
\hline$v=\mathbf{4}$ & 15297.5 & 15366.7 & 15371.2 & 15439.2 & 15518.2 & 15583.5 & 15737.2 & 15798.4 & 16026.7 & 16081.9 \\
\hline
\end{tabular}

TABLE 8. Relative (percent) difference between theoretical and experimental energies for the heavy molecules ${ }^{14} \mathrm{~N}_{2}$ and ${ }^{12} \mathrm{C}^{16} \mathrm{O}$. Negative values indicate theoretical energies that lie below experimental ones.

\begin{tabular}{|l|c|c|c|c|c|c|c|c|c|c|}
\hline & \multicolumn{2}{|c|}{$\boldsymbol{J = 0}$} & \multicolumn{2}{c|}{$\boldsymbol{J}=\mathbf{1}$} & \multicolumn{2}{c|}{$\boldsymbol{J}=\mathbf{2}$} & \multicolumn{2}{c|}{$\boldsymbol{J = 3}$} & \multicolumn{2}{c|}{$\boldsymbol{J = 4}$} \\
\hline & $\mathbf{N}_{\mathbf{2}}$ & $\mathbf{C O}$ & \multicolumn{1}{|c|}{$\mathbf{N}_{\mathbf{2}}$} & \multicolumn{1}{c|}{$\mathbf{C O}$} & \multicolumn{1}{|c|}{$\mathbf{N}_{\mathbf{2}}$} & $\mathbf{C O}$ & $\mathbf{N}_{\mathbf{2}}$ & $\mathbf{C O}$ & $\mathbf{N}_{\mathbf{2}}$ & $\mathbf{C O}$ \\
\hline $\boldsymbol{v}=\mathbf{0}$ & 0.08 & -0.05 & 0.09 & -0.04 & 0.09 & -0.05 & 0.09 & -0.05 & 0.10 & -0.05 \\
\hline $\boldsymbol{v}=\mathbf{1}$ & 0.06 & -0.06 & 0.06 & -0.06 & 0.06 & -0.06 & 0.06 & -0.05 & 0.06 & -0.05 \\
\hline $\boldsymbol{v}=\mathbf{2}$ & 0.02 & -0.06 & 0.02 & -0.06 & 0.02 & -0.06 & 0.02 & -0.06 & 0.03 & -0.06 \\
\hline $\boldsymbol{v}=\mathbf{3}$ & -0.02 & -0.06 & -0.02 & -0.06 & -0.02 & -0.06 & -0.02 & -0.06 & -0.02 & -0.06 \\
\hline $\boldsymbol{v}=\mathbf{4}$ & -0.08 & -0.07 & -0.08 & -0.06 & -0.08 & -0.07 & 0.04 & -0.06 & -0.08 & -0.06 \\
\hline
\end{tabular}

TABLE 9. Relative (percent) difference between theoretical and experimental energies for the light molecules ${ }^{1} \mathrm{H}^{19} \mathrm{~F}$ and ${ }^{1} \mathrm{H}^{2} \mathrm{H}$ (HD). Negative values indicate theoretical energies that lie below experimental ones.

\begin{tabular}{|l|c|c|c|c|c|c|c|c|c|c|}
\hline & \multicolumn{2}{|c|}{$\boldsymbol{J = 0}$} & \multicolumn{2}{c|}{$\boldsymbol{J}=\mathbf{1}$} & \multicolumn{2}{c|}{$\boldsymbol{J}=\mathbf{2}$} & \multicolumn{2}{c|}{$\boldsymbol{J}=\mathbf{3}$} & \multicolumn{2}{|c|}{$\boldsymbol{J}=\mathbf{4}$} \\
\hline & HF & HD & HF & HD & HF & HD & HF & HD & HF & \multicolumn{1}{|c|}{ HD } \\
\hline$v=\mathbf{0}$ & 0.39 & 0.35 & 0.37 & 0.34 & 0.34 & 0.31 & 0.29 & 0.29 & 0.18 & 0.26 \\
\hline $\boldsymbol{v}=\mathbf{1}$ & 0.17 & 0.06 & 0.16 & 0.06 & 0.12 & 0.07 & 0.00 & 0.08 & -0.27 & 0.09 \\
\hline$v=\mathbf{2}$ & 0.16 & -0.09 & 0.15 & -0.08 & 0.09 & -0.07 & -0.11 & -0.05 & -0.57 & -0.02 \\
\hline$v=\mathbf{3}$ & 0.19 & -0.25 & 0.18 & -0.24 & 0.09 & -0.22 & -0.20 & -0.19 & -0.86 & -0.16 \\
\hline$v=\mathbf{4}$ & 0.22 & -0.45 & 0.21 & -0.44 & 0.09 & -0.42 & -0.29 & -0.39 & -1.15 & -0.34 \\
\hline
\end{tabular}

One last interesting numerical result is presented. Theoretical values for spectroscopic constants of ${ }^{1} \mathrm{H}_{2}$ were obtained by fitting RSPT(6) energy data for ${ }^{1} \mathrm{H}_{2}$ to polynomials in $v$ and $J$ 
corresponding to truncated versions of (76-79). Thus, for example, the value of $\tilde{\alpha}_{e}$ in (76) is the coefficient of the $J(J+1)\left(v+\frac{1}{2}\right)$ term in such a polynomial. Mathematica's intrinsic function NonlinearRegress ${ }^{21}$ was used to perform the numerical fitting procedure, and the ${ }^{1} \mathrm{H}_{2}$ energy data from Table 2 for $0 \leq v \leq 3$ and $0 \leq J \leq 10$ were used as input (these energies, one will recall, differed from experiment by less than one percent). The theoretical spectroscopic constants obtained in this manner are listed in Table 10 alongside their experimental counterparts, which were taken from [32]. The numerical fit of the theoretical data has an estimated standard deviation of 1.24 waves per centimeter.

TABLE 10. Theoretical and experimental spectroscopic constants for ${ }^{1} \mathrm{H}_{2}$. Theoretical values were calculated by Mathematica to six significant digits, while experimental values were obtained from [32].

\begin{tabular}{|c|c|c|}
\hline $\begin{array}{c}\text { Spectroscopic } \\
\text { Constant }\end{array}$ & $\begin{array}{c}\text { Theoretical Value } \\
\text { [waves/centimeter] }\end{array}$ & $\begin{array}{c}\text { Experimental Value } \\
\text { [waves/centimeter] }\end{array}$ \\
\hline$\widetilde{\omega}_{e}$ & 4400.33 & 4401.213 \\
\hline$\widetilde{\omega}_{e} x_{e}$ & -122.135 & -121.336 \\
\hline$\widetilde{B}_{e}$ & 60.616 & 60.8530 \\
\hline$\tilde{\alpha}_{e}$ & -3.0655 & -3.0622 \\
\hline$\widetilde{D}_{e}$ & -0.0399509 & -0.0471 \\
\hline$\tilde{\mathrm{Y}}_{00}$ & 10.2031 & 8.93 \\
\hline
\end{tabular}

\section{CONCLusion}

Formula (57) provides a general expression for the Rayleigh-Schrödinger expansion of perturbed wave functions when a power series expansion of the molecular Hamiltonian is employed. This formula is valid in any application of Rayleigh-Schrödinger perturbation theory and complements the general perturbation energy formula obtained by Herbert [26]. In fact, these two formulas may be combined to yield the energy correction formula (67), which also holds for any application of Rayleigh-Schrödinger perturbation theory. In the context of vibrationalrotational analysis, however, Equation (67) may be simplified by writing the perturbed Hamiltonian matrices in terms of powers of the normal coordinate matrix.

${ }^{21}$ This function is contained in the package Statistics 'NonlinearFit`. 
In this report, such an analysis was presented for diatomic molecules. First, an appropriate Hamiltonian operator, exact within a certain radius of convergence, was derived [Equation (23)] and then written in perturbation-theoretical form as a power series whose individual terms are given by (25) and (27). Next, it was shown how an arbitrary element $\mathcal{H}_{\xi_{1}, \xi_{2}}^{(i)}$ of the $i$ th perturbed Hamiltonian matrix could be expressed in terms of normal coordinate matrix elements [Equation (40)]. Finally, Equation (40) was substituted into general formulas for Rayleigh-Schrödinger perturbation energies and expansion coefficients to yield equations specific to this application [(68) and (70), respectively]. Alternatively, one may forego derivation of (68) and (70) and proceed directly to implementation on Mathematica using Equations (58) and (63).

Using the packages RSPERTURB and DIATOMICVIBROT, Mathematica derived symbolic perturbation energy formulae which were then evaluated numerically for several test molecules. A procedure was given whereby the accuracy of such theoretical calculations may be estimated. However, the numerical results are not the significant accomplishment of this report; rather, these results serve only to demonstrate that computer algebra can successfully be used to implement high-order perturbation calculations that are much too laborious to be accomplished manually. In this regard, the most significant parts of this report are the RSPERTURB and DIATOMICVIBROT programs, for they allow anyone-quantum chemist or experimentalist - to take advantage of arbitrary-order perturbation theory (RSPERTURB) to solve diatomic vibrational-rotational analysis problems (DIATOMICVIBRoT) using Mathematica.

Although derivation of symbolic high-order perturbation formulae requires a significant amount of computation time due to the nature of the Mathematica software package, significant improvements in algorithm efficiency (as discussed in Section IX) will be made before this analysis is applied to polyatomic molecules. Even so, the methods presented herein may already be more efficient than numerical procedures, for the calculations performed by Mathematica result in symbolic formulae that express energy corrections in terms of arbitrary universal and molecular constants. Once the RSPT(6) formulae in Appendices C-E were derived, for example, energy corrections through sixth order were rapidly calculated for all of the molecules in Section X simply by substitution of appropriate constants. Because of their symbolic nature, once published the formulae in Appendices C-E need never be derived again.

Computer algebra has proven to be an effective tool in the application of perturbation theory to problems in quantum mechanics. In future work, the procedures presented here will be 
applied to vibrational-rotational analysis problems involving polyatomic molecules. Many of the results presented in this report and in [26] are quite general and can readily be extended to polyatomic molecules. The major difficulties in such an application are derivation of an appropriate Hamiltonian, segmentation of that Hamiltonian into a power series consisting of separate perturbation terms, and derivation of explicit forms for the polyatomic vibrationalrotational wave functions. Once these obstacles are overcome, however, the remainder of the treatment should be analogous to that presented in this report 


\section{APPENDIX A \\ The Mathematica Package RSperturb}

Note that the function Qdelta in this package is a slightly modified version of the code authored by Dudas et al. [9].

\section{BeginPackage [ "Rsperturb" "]}

(* Two variables must be specified at the beginning of each session: HighestState is the numerical value of the highest quantum state for which formulae are to be derived, while Highestorder is the highest order of correction for which formulae will be derived. Specifying larger values of these variables than are necessary will not adversely affect the output, although the derivations may require more CPU time than would otherwise be necessary. *)

Energy: : usage =

"Energy $[n, v]$ derives the nth-order energy correction formula for quantum state $v$ using the most general form of the perturbation energy formula (as derived by Herbert [26])."

Egy: : usage $=$

"Egy $[i, v]$ is the ith-order energy correction for quantum state $v$ as referenced by the function Energy $[n, v]$. This notation allows a recursive formula for Energy $[n, v]$ to be generated without explicit evaluation of the lower-order energies on which it depends."

KDelta: :usage = "The Kronecker delta function."

SumStates: : usage $=$

"Sumstates[expr, $\{v, v$ ", vmax $\}$ ] sums expr over the quantum numbers from $v$ to vmax, skipping $v=v^{\prime}$."

Qdelta: : usage $=$

"Qdelta[n] derives expressions for the elements of the matrices $Q^{1}, Q^{2}, \ldots, Q^{n}$. This is a slightly modified version of the function published in [9]; this version incorporates Kronecker delta functions."

Generatecoefficients: : usage =

"Generatecoefficients $[n, v]$ returns a matrix containing all of the RayleighSchrödinger expansion coefficients necessary to expand the nth perturbed wave function for quantum state $v$. This function is useful for obtaining the explicit form of the wave function as a linear combination of the zeroth-order wave functions."

RSCoefficients1: : usage = "RSCoefficients $1[n, j,\{v\}]$ generates the $n$ th-order Rayleigh-Schrödinger expansion coefficient associated with quantum state $j$ such that the summation excludes $j=v$. This function is intended to be called by other functions."

RSCoefficients $2:$ : usage =

"Usage is the same as the function RScoefficients1; both functions are necessary for matrix elements that require two different expansion coefficients."

Psi: : usage $=$

"Psi[n,v] is the nth-order perturbed wave function for quantum state $v . "$ 
$\mathrm{H}:$ : usage $=$

"H[n] is the nth-order perturbed Hamiltonian operator."

$k:$ : usage $=$

"k[i] is the symbolic representation of the ith molecular force constant."

Int: : usage $=$

"Int [Psi $[n 1, v 1], H[x], P s i[n 2, v 2]]$ is a quantum-mechanical integra]

involving a perturbed Hamiltonian operator. If $n 1=0$ and $n 2=0$, then

this function is a Hamiltonian matrix element as defined in Equation (34)."

Int: : usage $=$

"Int $[\operatorname{Ps} i[n 1, v 1]$, Psi $[n 2, v 2]]$ is an overlap integral."

(* Other symbols appearing in the symbolic output are:

a, which represents the constant $\alpha$ in (15);

ke, the equilibrium molecular force constant;

ve, the harmonic frequency as defined in (16);

h, Planck's constant;

$\mathrm{Be}$, the equilibrium rotational constant (19); and

$\mathrm{Re}$, the equilibrium internuclear separation. *)

Highest $=$ HighestState $+3+$ Sum[index, $\{$ index, 3, Highestorder +1$\}]$;

$A A=\operatorname{Array}[A$, Highest $] ;$

$\mathrm{BB}=$ Array $[\mathrm{B}$, Highest $]$;

Unprotect [Part];

Attributes [KDelta] = \{Orderless $\}$;

KDelta[a_, $\left.b_{-}\right]:=$Which $[a==b, 1, a !=b, 0] \cdot / ; a==b|| a !=b$

Sumstates [expr_, \{var_, hole_, $\left.\left.\max _{-}\right\}\right]:=$

Sum[expr, $\{v a r,-\max$, hole $-\overline{1}\}]+\operatorname{sum}[\operatorname{expr},\{$ var, hole $+1, \max \}]$

(* Expansion of perturbed wave functions in Hamiltonian matrix elements using Equation (49) *)

Int $/:$ Int [Psi[n1_Integer, v1_], h:_H, Psi[n2_Integer, v2_] ] :=

Block $[\{$ pdt $\}$,

pdt $=$ SumStates $[R S C o e f f i c i e n t s 1[n 2, i,\{v 2\}] *$

Int [Psi[0, j], h, Psi[0, i]], \{i, v2, Highest $\}$;

Sumstates [RSCoefficients2[n1, j, $\{\mathrm{v} 1\}]$ pdt, $\{j, v 1$, Highest $\}]$

] $/ ;(n 1>0) \& \&(n 2>0)$

Int /: Int [Psi[n1_Integer, v1_], h:_H, Psi[n2_Integer, v2_]] :=

Sumstates [RSCoefficients1[n1, j, \{v1\}] Int [Psi[0, j], $\bar{h}, \operatorname{Psi}[n 2, v 2]]$,

$\{j, v 1$, Highest $\}] / ;(\mathrm{n} 2==0)$ \&\& $(\mathrm{n} 1>0)$

Int $/$ : Int [Psi[n1_Integer, v1_], h:_H, Psi[n2_Integer, v2_] ] :=

Sumstates [RSCoefficients2[n2, j, [v2\}] Int [Psi[n1, v1], h, Psi[0, j]],

$\{j, \mathrm{v} 2, \mathrm{Highest}\}] / ;(\mathrm{n} 1==0)$ \&\& $(\mathrm{n} 2>0)$

(* Expansion of perturbed wave functions in overlap integrals using Equation $(50) *)$

Int /: Int [Psi[n1_Integer, v_], Psi[n2_Integer, v_] ] :=

Sumstates [RSCoefficientsi[n1, j, $\{v\}]$ *

RSCoefficients2[n2, j, $\{v\}],\{j, v$, Highest $\}] / ;(n 1>0)||(n 2>0)$

(* Hermitian property of quantum-mechanical integrals *)

Int /: Int [Psi[n1_, v1_], h:_H, Psi[n2_, v2_] ] :=

Int[Psi[n2, v2], h, Psi[n1, v1]]/; Order[Psi[n1, v1], Psi[n2, v2]] == -1

Int /: Int [Psi[n1_, v1_], Psi[n2_, v2_]] :=

Int [Psi[n2, v2], Psi[n1, v1]] /; Order[Psi[n1, v1], Psi[n2, v2]] == -1

(* Orthogonality of zeroth-order wave functions *)

Int /: Int $\left[\mathrm{Psi}\left[0, \mathrm{v} 1_{-}\right], \operatorname{Psi}\left[0, v 2_{-}\right]\right]:=\operatorname{KDelta}[\mathrm{v} 1, \mathrm{v} 2]$

Energy $\left[n_{-}, v_{-}\right]:=$

Block [ term1, term2, term3, term4, term5, span, ii, jj\}, 
If $[$ EvenQ $[n]==$ True, $\operatorname{span}=n / 2, \operatorname{span}=(n-1) / 2] ;$ $\operatorname{term} 1=\operatorname{Sum}[(2-\operatorname{kDelta}[n, 2$ ii] $) \operatorname{Int}[\operatorname{Psi}[j j-1, V]$,

$H[n-i i-j j+1], \operatorname{Ps} i[i i, V]],\{j j, 1, \operatorname{span}\},\{i i, j j, \operatorname{span}\}] ;$

term2 = Sum[Int[Psi[ii, V], H[n - 2 ii], Psi[ii, V]],

$\{i, 0, \operatorname{span}-1\}]$;

term3 $=\operatorname{Sum}[(2-\operatorname{kDelta}[n, 2 i i]) \operatorname{Egy}[n-i i-j j+1, V]$

Int $[\operatorname{Ps} i[j j-1, V], \operatorname{Ps} i[i i, V]],\{j j, 2, \operatorname{span}\},\{i i, j j, \operatorname{span}\}]$;

term4 = Sum[Egy[n - 2 ii, V]*

Int [Psi[ii, V], Psi[ii, V]], \{ii, 1, span - 1\}];

term5 = KDelta [n, 2 span + 1] (Int [Psi[span, V], H[1], Psi[span, V]] Egy[1, V] Int [Psi[span, V], Psi[span, V]] (1 - KDelta[1, n]));

term1 + term2 - term3 - term4 + term5

] $1 ; \mathrm{n}>0$

Qdelta[n_Integer] :=

$\operatorname{Block}[\{z z, u, y y, g g, d r, y, z e d, i i, j j, y m\}$,

$\mathrm{ss}=\operatorname{Array}[\mathrm{s},\{16$, Highest $\}] ;$

$\operatorname{ss}[[1,1]]=(($ qnum +1$) /(2$ a $)) \wedge(1 / 2)$;

ss $[\{1,2]]=(\text { qnum } /(2 \text { a }))^{\wedge}(1 / 2)$;

$\mathrm{u}=2$;

While $[u<n+1, y Y=u-1$;

$\operatorname{ss}[[u, 1]]=(\operatorname{ss}[[y Y, 1]] /$ qnum $\rightarrow$ qnum +1$)$ ss $[[1,1]]$;

zed $=2$;

$g g=u+1$

While[zed < gg, $z z=$ zed -1 ;

$\operatorname{ss}[[u, z e d]]=(s s[[y y, z z]] /$ qnum $\rightarrow$ qnum -1$)$ ss $[[1,2]]+$

zed ++$]$

(ss[ [yy,zed] ] /. qnum $\rightarrow$ qnum +1 ) ss $[[1,1]]$;

$\mathrm{ym}=\mathrm{u}+1$

$\mathrm{ss}[[\mathrm{u}, \mathrm{ym}]]=(\mathrm{ss}[\mathrm{yY}, \mathrm{u}]] /$ gnum $\rightarrow$ gnum -1$) \mathrm{ss}[[1,2]]$;

$\mathrm{u++}$;

$\mathrm{dr}=$ Highest $-1-\mathrm{n}$;

Drop [ss [ [n]], $-d r]$;

qdel = Array [qd, $\mathrm{n}]$;

Do[qdel[[ii]] = Sum[ss[[ii, jj]]

KDelta[qp, qnum $+i i-2(j j-1)\},\{j j, i i+1\}],\{i i, n\}] ;$ qdel $[\mathrm{n}]]]$

GenerateCoefficients[ord_Integer, state_Integer] :=

Block [ (counter, index 1 , index 2 , temp, count 2 \},

temp = Array $[t$, ord $]$;

coefficients = Array $[\mathrm{cc},\{$ ord, ord +3$\}]$;

Do[term $1=-\operatorname{Int}[\operatorname{Psi}[0, \mathrm{~m}], \mathrm{H}[$ counter $]$, Psi[0, state] $] /$

(h ve (m - state));

term2 = $1 /(\mathrm{h}$ ve $(m-$ state $))$ Sum[Egy[counter - index1, state] * temp [ [index1] ], \{index1, counter - 1\}];

term $3=-1 /($ h ve $(m-s t a t e))$ Sum [Sum [ (temp [ [index1] $] /\{m \rightarrow$ index 2$\}) \operatorname{Int}[\mathrm{Psi}[0, \mathrm{~m}], \mathrm{H}[$ counter - index 1$], \operatorname{Psi}[0$, index 2$]]$, \{index 2 , state - counter + index $1-3$, state $-1,2\}]+$ Sum[(temp [[index1]] /. \{m $\rightarrow$ index 2$\})$ Int [Psi $[0, m]$,

$\mathrm{H}[$ counter - index1], Psi[0, index2]], \{index2, state + counter index $1+3$, state $+1,-2\}]$. \{index 1 , counter -1$\}]$;

temp $[$ counter] $]=\operatorname{term} 1+\operatorname{term} 2+\operatorname{term} 3$;

Do $[$ coefficients $[$ counter, count 2$]]=$ temp $[$ counter $]] / . \mathrm{m} \rightarrow$ $(-2+$ state - counter $+2 *($ count $2-1))$,

$\{$ count2, Floor $[$ (counter +3$) / 2\}\}]$;

Do [coefficients [ [counter, count2] $]=\operatorname{temp}[[$ counter $]] / . \mathrm{m} \rightarrow$ $(-2+$ state - counter $+2 *($ count $2-1))$,

\{count2, Ceiling [(counter +3$) / 2]+1$, counter +3$\}]$;

If [Eveng [ counter] $==$ True,

coefficients [ counter, Ceiling [(counter +3$) / 2]]]]$,

\{counter, ord\}];

coefficients 


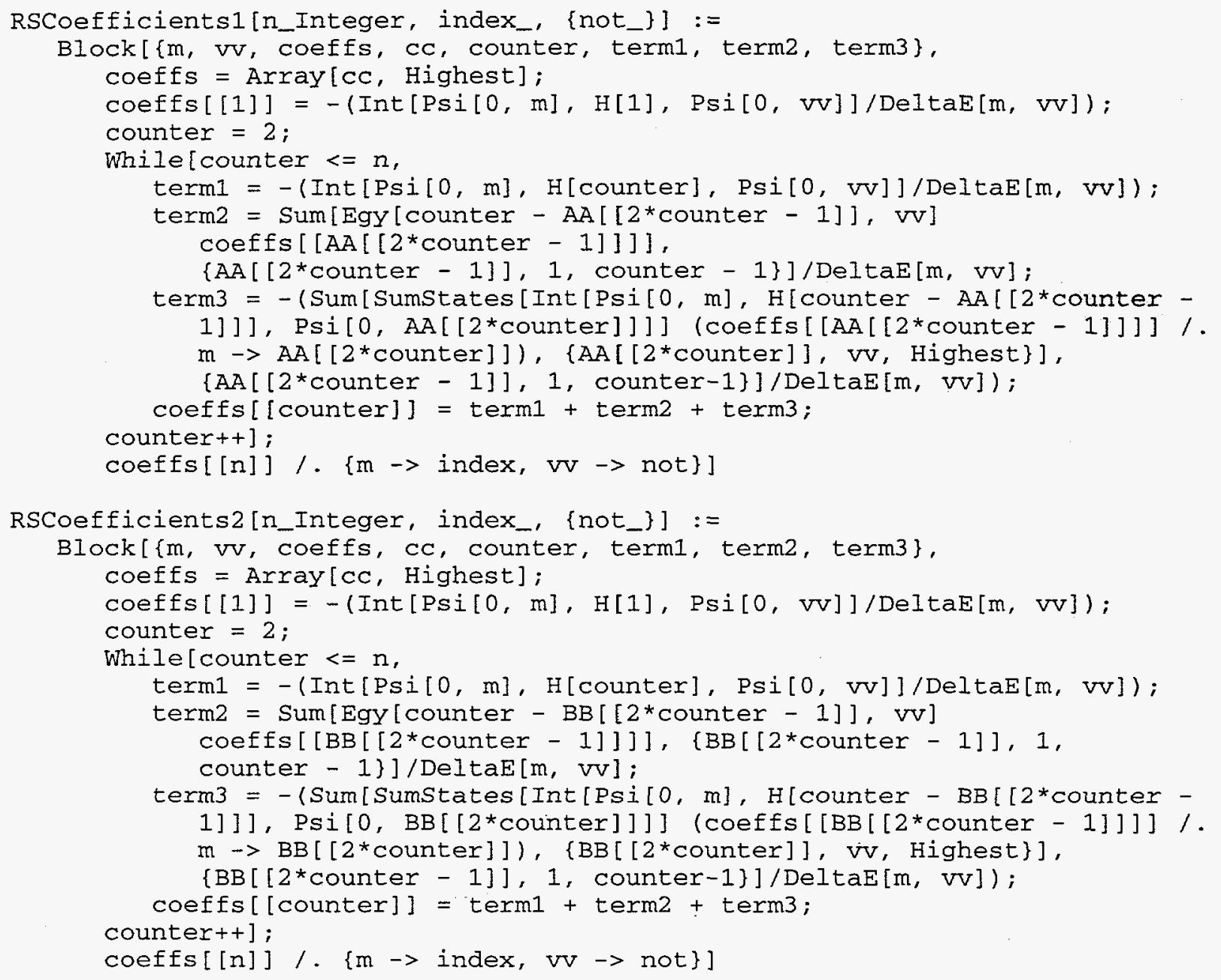

EndPackage [ ] 
APPENDIX B

The Mathematica PaCKage DiatomicVibRot

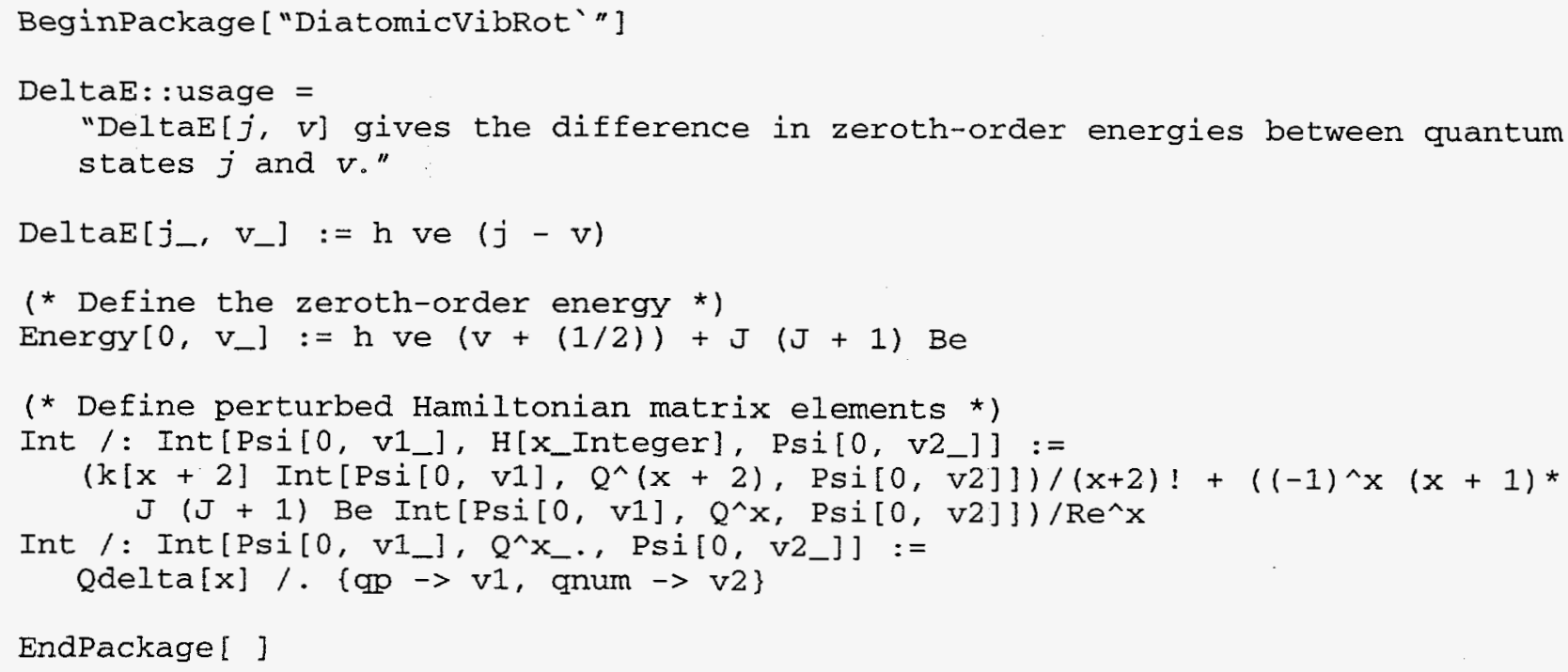




\section{SECOND-ORDER CORRECTION FormulaE}

For each vibrational state, $E^{(2)}$ is a linear combination of the purely vibrational terms, purely rotational terms, and vibration-rotation coupling terms listed below.

$$
\begin{array}{ll}
\text { VibRATIONAL TERMs: } & A_{2}^{(\mathrm{v})}, B_{2}^{(\mathrm{v})} \\
\text { RotATIONAL TERMS: } & A_{2}^{(\mathrm{r})}, B_{2}^{(\mathrm{r})} \\
\text { COUPLING TERMS: } \quad A_{2}^{(\mathrm{v}-\mathrm{r})}, B_{2}^{(\mathrm{v}-\mathrm{r})}
\end{array}
$$

Using lower-case letters to represent the integer coefficients of a linear combination, one can write the complete second-order energy correction as

$$
\begin{aligned}
E_{v, J}^{(2)} & =a_{2}^{(\mathrm{v})} A_{2}^{(\mathrm{v})}+b_{2}^{(\mathrm{v})} B_{2}^{(\mathrm{v})}+a_{2}^{(\mathrm{r})} A_{2}^{(\mathrm{r})}+b_{2}^{(\mathrm{r})} B_{2}^{(\mathrm{r})}+a_{2}^{(\mathrm{v}-\mathrm{r})} A_{2}^{(\mathrm{v}-\mathrm{r})}+\mathrm{b}_{2}^{(\mathrm{v}-\mathrm{r})} B_{2}^{(\mathrm{v}-\mathrm{r})} \\
& =a_{2}^{(\mathrm{v})} A_{2}^{(\mathrm{v})}+\mathrm{b}_{2}^{(\mathrm{v})} B_{2}^{(\mathrm{v})}+\mathrm{a}_{2}^{(\mathrm{v}-\mathrm{r})} A_{2}^{(\mathrm{v}-\mathrm{r})}+\mathrm{b}_{2}^{(\mathrm{v}-\mathrm{r})} B_{2}^{(\mathrm{v}-\mathrm{r})}+\Re_{2},
\end{aligned}
$$

where the coefficients depend upon the vibrational state $v$. Values of the linear combination coefficients for the first eleven vibrational states are listed in Table C-1.

TABLE C-1. Linear combination coefficients for $E^{(2)}$.

\begin{tabular}{|c|c|c|c|c|}
\hline$v$ & $a_{2}^{(v)}$ & $b_{2}^{(v)}$ & $a_{2}^{(v-r)}$ & $b_{2}^{(v-r)}$ \\
\hline 0 & 11 & 1 & 3 & 1 \\
\hline 1 & 71 & 5 & 9 & 3 \\
\hline 2 & 191 & 13 & 15 & 5 \\
\hline 3 & 371 & 25 & 21 & 7 \\
\hline 4 & 611 & 41 & 27 & 9 \\
\hline 5 & 911 & 61 & 33 & 11 \\
\hline 6 & 1271 & 85 & 39 & 13 \\
\hline 7 & 1691 & 113 & 45 & 15 \\
\hline 8 & 2171 & 145 & 51 & 17 \\
\hline 9 & 2711 & 181 & 57 & 19 \\
\hline 10 & 3311 & 221 & 63 & 21 \\
\hline
\end{tabular}


$\Re_{2}$ in (79) is the second-order constant of pure rotation and is defined as the sum of the two purely rotational terms listed above (since these terms are independent of the vibrational state, the coefficients $\mathrm{a}_{2}^{(\mathrm{r})}$ and $\mathrm{b}_{2}^{(\mathrm{r})}$ will be the same for each value of $v$ and can thus be incorporated into a single constant $\Re_{2}$ which is independent of vibrational state). Capital letters in the energy formula (79) represent collections of universal and molecular constants, which have the form

$$
\frac{J^{\ell_{1}}(J+1)^{\ell_{2}} B_{e}^{\ell_{3}} k_{3}^{\ell_{4}} k_{4}^{\ell_{5}}}{\Omega \alpha^{\ell_{6}} R_{e}^{\ell_{7}}\left(h v_{e}\right)^{\ell_{8}}},
$$

where $\Omega, \ell_{1}, \ell_{2}, \ldots, \ell_{8}$ are integers whose values are listed in Table $\mathrm{C}-2$ for all vibrational and coupling terms. Lastly, the second-order constant of pure rotation is

$$
\Re_{2}=\frac{-2 J^{2}(J+1)^{2} B_{e}^{2}}{\alpha R_{e}^{2} h v_{e}} .
$$

TABLE C-2. Symbolic term factors (80) for $E^{(2)}$.

\begin{tabular}{|c|c|c|c|c|c|c|c|c|c|}
\hline & $\ell_{1}$ & $\ell_{2}$ & $\ell_{3}$ & $\ell_{4}$ & $\ell_{5}$ & $\ell_{6}$ & $\ell_{7}$ & $\ell_{8}$ & $\Omega$ \\
\hline$A_{2}^{(\mathrm{v})}$ & 0 & 0 & 0 & 2 & 0 & 3 & 0 & 1 & -288 \\
\hline$B_{2}^{(\mathrm{v})}$ & 0 & 0 & 0 & 0 & 1 & 2 & 0 & 0 & 32 \\
\hline$A_{2}^{(\mathrm{v}-\mathrm{r})}$ & 1 & 1 & 1 & 0 & 0 & 1 & 2 & 0 & 2 \\
\hline$B_{2}^{(\mathrm{v}-\mathrm{r})}$ & 1 & 1 & 1 & 1 & 0 & 2 & 1 & 1 & 2 \\
\hline
\end{tabular}




\section{APPENDIX D}

\section{FOURTH-ORdER CORRECTION FormulaE}

Using the notation developed in Appendix C, one can express the fourth-order energy correction formulae for all vibrational and rotational states in the form of a linear combination of symbolic constants:

$$
E_{v, J}^{(4)}=a_{4}^{(v)} A_{4}^{(v)}+\cdots+e_{4}^{(v)} E_{4}^{(v)}+a_{4}^{(v-r)} A_{4}^{(v-r)}+\cdots+o_{4}^{(v-r)} O_{4}^{(v-r)}+\Re_{4} .
$$

The fourth-order constant of pure rotation in Equation (82) is

$$
\Re_{4}=\frac{12 J^{3}(J+1)^{3} B_{e}^{3}}{\alpha^{2} R_{e}^{4}\left(h v_{e}\right)^{2}}+\frac{4 J^{3}(J+1) B_{e}^{3} k_{3}}{\alpha^{3} R_{e}^{3}\left(h v_{e}\right)^{3}}\left(3 J^{2}-2 J+3\right) \text {. }
$$

Notice that the final term in $\Re_{4}$ cannot be factored into integer powers of $J$ or $J+1$, as predicted by Darling and Dennison [42]. Values for the coefficients in (82) are given in Tables D-1 and D-2.

TABLE D-1. Linear combination coefficients for $E^{(4)}$.

\begin{tabular}{|c|c|c|c|c|c|c|c|c|c|c|}
\hline$v$ & $\mathrm{a}_{4}^{(v)}$ & $\mathrm{b}_{4}^{(v)}$ & $\mathrm{c}_{4}^{(\mathrm{v})}$ & $\mathrm{d}_{4}^{(\mathrm{v})}$ & $\mathrm{e}_{4}^{(v)}$ & $\mathrm{a}_{4}^{(\mathrm{v}-\mathrm{r})}$ & $\mathrm{b}_{4}^{(\mathrm{v}-\mathrm{r})}$ & $\mathrm{c}_{4}^{(v-r)}$ & $\mathrm{a}_{4}^{(\mathrm{v}-\mathrm{r})}$ & $\mathrm{e}_{4}^{(\mathrm{v}-\mathrm{r})}$ \\
\hline 0 & 155 & 19 & 70 & 13 & 1 & 15 & 57 & 57 & 9 & 9 \\
\hline 1 & 1875 & 207 & 550 & 123 & 7 & 75 & 171 & 171 & 27 & 27 \\
\hline 2 & 7825 & 845 & 2050 & 485 & 25 & 195 & 285 & 285 & 45 & 45 \\
\hline 3 & 20825 & 2233 & 5250 & 1267 & 63 & 375 & 399 & 399 & 63 & 63 \\
\hline 4 & 43695 & 4671 & 10830 & 2637 & 129 & 615 & 513 & 513 & 81 & 81 \\
\hline 5 & 79255 & 8459 & 19470 & 4763 & 231 & 915 & 627 & 627 & 99 & 99 \\
\hline 6 & 130325 & 13897 & 31850 & 7813 & 377 & 1275 & 741 & 741 & 117 & 117 \\
\hline 7 & 199725 & 21285 & 48650 & 11955 & 575 & 1695 & 855 & 855 & 135 & 135 \\
\hline 8 & 290275 & 30923 & 70550 & 17357 & 833 & 2175 & 969 & 969 & 153 & 153 \\
\hline 9 & 404795 & 43111 & 98230 & 24187 & 1159 & 2715 & 1083 & 1083 & 171 & 171 \\
\hline 10 & 546105 & 58149 & 132370 & 32613 & 1561 & 3315 & 1197 & 1197 & 189 & 189 \\
\hline
\end{tabular}


TABLE D-2. Linear combination coefficients for $E^{(4)}$.

\begin{tabular}{|c|c|c|c|c|c|c|c|c|c|c|}
\hline$v$ & $\mathrm{f}_{4}^{(\mathrm{v}-\mathrm{r})}$ & $\mathrm{g}_{4}^{(\mathrm{v}-\mathrm{r})}$ & $\mathrm{h}_{4}^{(\mathrm{v}-\mathrm{r})}$ & $i_{4}^{(\mathrm{v}-\mathrm{r})}$ & $j_{4}^{(\mathrm{v}-\mathrm{r})}$ & $\mathrm{k}_{4}^{(\mathrm{v}-\mathrm{r})}$ & $\mathrm{I}_{4}^{(\mathrm{v}-\mathrm{r})}$ & $\mathrm{m}_{4}^{(\mathrm{v}-\mathrm{r})}$ & $\mathrm{n}_{4}^{(\mathrm{v}-\mathrm{r})}$ & $\mathrm{o}_{4}^{(\mathrm{v}-\mathrm{r})}$ \\
\hline 0 & 11 & 3 & 3 & 11 & 11 & 1 & 1 & 3 & 31 & 1 \\
\hline 1 & 71 & 9 & 9 & 71 & 71 & 3 & 3 & 15 & 187 & 5 \\
\hline 2 & 191 & 15 & 15 & 191 & 191 & 5 & 5 & 39 & 499 & 13 \\
\hline 3 & 371 & 21 & 21 & 371 & 371 & 7 & 7 & 75 & 967 & 25 \\
\hline 4 & 611 & 27 & 27 & 611 & 611 & 9 & 9 & 123 & 1591 & 41 \\
\hline 5 & 911 & 33 & 33 & 911 & 911 & 11 & 11 & 183 & 2371 & 61 \\
\hline 6 & 1271 & 39 & 39 & 1271 & 1271 & 13 & 13 & 255 & 3307 & 85 \\
\hline 7 & 1691 & 45 & 45 & 1691 & 1691 & 15 & 15 & 339 & 4399 & 113 \\
\hline 8 & 2171 & 51 & 51 & 2171 & 2171 & 17 & 17 & 435 & 5647 & 145 \\
\hline 9 & 2711 & 57 & 57 & 2711 & 2711 & 19 & 19 & 543 & 7051 & 181 \\
\hline 10 & 3311 & 63 & 63 & 3311 & 3311 & 21 & 21 & 663 & 8611 & 221 \\
\hline
\end{tabular}

The fourth-order symbolic constants have the form

$$
\frac{J^{\ell_{1}^{\prime}}(J+1)^{\ell_{2}^{\prime}} B_{e}^{\ell_{3}^{\prime}} k_{3}^{\ell_{4}^{\prime}} k_{4}^{\ell_{5}^{\prime}} k_{5}^{\ell_{6}^{\prime}} k_{6}^{\ell_{7}^{\prime}}}{\Omega^{\prime} \alpha^{\ell_{8}^{\prime}} R_{e}^{\ell_{9}^{\prime}}\left(h v_{e}\right)^{\ell_{10}^{\prime}}} ;
$$

the values of $\Omega^{\prime}$ and $\ell_{1}^{\prime}$ through $\ell_{10}^{\prime}$ for eleven vibrational states are listed in Tables D-3 and D-4.

TABLE D-3. Symbolic term factors (84) for $E^{(4)}$.

\begin{tabular}{|l|c|c|c|c|c|c|c|c|c|c|c|}
\hline & $\ell_{1}^{\prime}$ & $\ell_{2}^{\prime}$ & $\ell_{3}^{\prime}$ & $\ell_{4}^{\prime}$ & $\ell_{5}^{\prime}$ & $\ell_{6}^{\prime}$ & $\ell_{7}^{\prime}$ & $\ell_{8}^{\prime}$ & $\ell_{9}^{\prime}$ & $\ell_{10}^{\prime}$ & $\Omega^{\prime}$ \\
\hline$A_{4}^{(\mathrm{v})}$ & 0 & 0 & 0 & 4 & 0 & 0 & 0 & 6 & 0 & 3 & -13824 \\
\hline$B_{4}^{(\mathrm{v})}$ & 0 & 0 & 0 & 2 & 1 & 0 & 0 & 5 & 0 & 2 & 768 \\
\hline$C_{4}^{(\mathrm{v})}$ & 0 & 0 & 0 & 0 & 2 & 0 & 0 & 4 & 0 & 1 & -1536 \\
\hline$D_{4}^{(\mathrm{v})}$ & 0 & 0 & 0 & 1 & 0 & 1 & 0 & 4 & 0 & 1 & -1152 \\
\hline$E_{4}^{(\mathrm{v})}$ & 0 & 0 & 0 & 0 & 0 & 0 & 1 & 3 & 0 & 0 & 384 \\
\hline$A_{4}^{(\mathrm{v}-\mathrm{r})}$ & 1 & 1 & 1 & 0 & 0 & 0 & 0 & 2 & 4 & 0 & 4 \\
\hline
\end{tabular}


TABLE D-4. Symbolic term factors (84) for $E^{(4)}$.

\begin{tabular}{|l|c|c|c|c|c|c|c|c|c|c|c|}
\hline & $\ell_{1}^{\prime}$ & $\ell_{2}^{\prime}$ & $\ell_{3}^{\prime}$ & $\ell_{4}^{\prime}$ & $\ell_{5}^{\prime}$ & $\ell_{6}^{\prime}$ & $\ell_{7}^{\prime}$ & $\ell_{8}^{\prime}$ & $\ell_{9}^{\prime}$ & $\ell_{10}^{\prime}$ & $\Omega^{\prime}$ \\
\hline$B_{4}^{(\mathrm{v}-\mathrm{r})}$ & 2 & 1 & 2 & 0 & 0 & 0 & 0 & 2 & 4 & 1 & -4 \\
\hline$C_{4}^{(\mathrm{v}-\mathrm{r})}$ & 3 & 1 & 2 & 0 & 0 & 0 & 0 & 2 & 4 & 1 & -4 \\
\hline$D_{4}^{(\mathrm{v}-\mathrm{r})}$ & 2 & 1 & 2 & 1 & 0 & 0 & 0 & 3 & 3 & 2 & -2 \\
\hline$E_{4}^{(\mathrm{v}-\mathrm{r})}$ & 3 & 1 & 2 & 1 & 0 & 0 & 0 & 3 & 3 & 2 & -2 \\
\hline$F_{4}^{(\mathrm{v}-\mathrm{r})}$ & 1 & 1 & 1 & 1 & 0 & 0 & 0 & 3 & 3 & 1 & 6 \\
\hline$G_{4}^{(\mathrm{v}-\mathrm{r})}$ & 2 & 1 & 2 & 2 & 0 & 0 & 0 & 4 & 2 & 3 & -4 \\
\hline$H_{4}^{(\mathrm{v}-\mathrm{r})}$ & 3 & 1 & 2 & 2 & 0 & 0 & 0 & 4 & 2 & 3 & -4 \\
\hline$I_{4}^{(\mathrm{v}-\mathrm{r})}$ & 1 & 1 & 1 & 2 & 0 & 0 & 0 & 4 & 2 & 2 & 24 \\
\hline$J_{4}^{(\mathrm{v}-\mathrm{r})}$ & 1 & 1 & 1 & 3 & 0 & 0 & 0 & 5 & 1 & 3 & 72 \\
\hline$K_{4}^{(\mathrm{v}-\mathrm{r})}$ & 2 & 1 & 2 & 0 & 1 & 0 & 0 & 3 & 2 & 2 & 2 \\
\hline$L_{4}^{(\mathrm{v}-\mathrm{r})}$ & 3 & 1 & 2 & 0 & 1 & 0 & 0 & 3 & 2 & 2 & 2 \\
\hline$M_{4}^{(\mathrm{v}-\mathrm{r})}$ & 1 & 1 & 1 & 0 & 1 & 0 & 0 & 3 & 2 & 1 & -16 \\
\hline$N_{4}^{(\mathrm{v}-\mathrm{r})}$ & 1 & 1 & 1 & 1 & 1 & 0 & 0 & 4 & 1 & 2 & -144 \\
\hline$O_{4}^{(\mathrm{v}-\mathrm{r})}$ & 1 & 1 & 1 & 0 & 0 & 1 & 0 & 3 & 1 & 1 & 16 \\
\hline
\end{tabular}




\section{APPENDIX E \\ SIXTH-ORDER CORRECTION FORMULAE}

The sixth-order correction formulae are given by the linear combination

$$
E_{v, J}^{(6)}=a_{6}^{(v)} A_{6}^{(v)}+\cdots+k_{6}^{(v)} K_{6}^{(v)}+a_{6}^{(v-r)} A_{6}^{(v-r)}+\cdots+11 I_{6}^{(v-r)} L L L_{6}^{(v-r)}+\Re_{6} .
$$

The sixth-order constant of pure rotation $\Re_{6}$ is

$$
\Re_{6}=\frac{2 B_{e}^{4} J^{4}(J+1)^{4} k_{4}}{3 \alpha^{4} R_{e}^{4}\left(h v_{e}\right)^{4}}-\frac{104 B_{e}^{4} J^{4}(J+1)^{4}}{\alpha^{3} R_{e}^{6}\left(h v_{e}\right)^{3}}-\frac{24 B_{e}^{4} J^{4}(J+1)^{4} k_{3}}{\alpha^{4} R_{e}^{5}\left(h v_{e}\right)^{4}}-\frac{2 B_{e}^{4} J^{4}(J+1)^{4} k_{3}^{2}}{\alpha^{5} R_{e}^{4}\left(h v_{e}\right)^{5}},
$$

while each sixth-order symbolic constant has the form

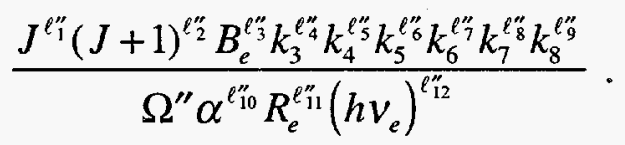

The coefficients of the linear combination (85) are given in Tables E-1 through E-6, while the symbolic term factors (87) are listed in Tables E-7 through E-9.

TABLE E-1. Linear combination coefficients for $E^{(6)}$.

\begin{tabular}{|c|c|c|c|c|c|c|c|c|}
\hline$v$ & $\mathrm{a}_{6}^{(\mathrm{v})}$ & $\mathrm{b}_{6}^{(\mathrm{v})}$ & $\mathrm{c}_{6}^{(\mathrm{v})}$ & $\mathrm{d}_{6}^{(\mathrm{v})}$ & $\mathrm{e}_{6}^{(\mathrm{v})}$ & $\mathrm{f}_{6}^{(\mathrm{v})}$ & $\mathrm{g}_{6}^{(\mathrm{v})}$ & $\mathrm{h}_{6}^{(\mathrm{v})}$ \\
\hline 0 & 39709 & 15169 & 11827 & 37 & 4517 & 237 & 449 & 323 \\
\hline 1 & 827539 & 289039 & 198565 & 435 & 77207 & 3472 & 5769 & 4745 \\
\hline 2 & 5181319 & 1767379 & 1170721 & 2231 & 456947 & 19602 & 31529 & 26621 \\
\hline 3 & 18657289 & 6311389 & 4125655 & 7425 & 1612457 & 67947 & 107969 & 92015 \\
\hline 4 & 49589809 & 16712869 & 10859407 & 19017 & 4246817 & 177487 & 280449 & 240023 \\
\hline 5 & 109091359 & 36694219 & 23766697 & 41007 & 9297467 & 386862 & 609449 & 522773 \\
\hline 6 & 211052539 & 70908439 & 45840925 & 78395 & 17936207 & 744372 & 1170569 & 1005425 \\
\hline 7 & 372142069 & 124939129 & 80674171 & 137181 & 31569197 & 1307977 & 2054529 & 1766171 \\
\hline 8 & 611806789 & 205300489 & 132457195 & 224365 & 51836957 & 2145297 & 3367169 & 2896235 \\
\hline 9 & 952271659 & 319437319 & 205979437 & 347947 & 80614367 & 3333612 & 5229449 & 4499873 \\
\hline 10 & 1418539759 & 475725019 & 306629017 & 516927 & 120010667 & 4959862 & 7777449 & 6694373 \\
\hline
\end{tabular}


TABLE E-2. Linear combination coefficients for $E^{(6)}$.

\begin{tabular}{|c|c|c|c|c|c|c|c|c|c|c|}
\hline$v$ & $i_{6}^{(v)}$ & $j_{6}^{(v)}$ & $k_{6}^{(v)}$ & $a_{6}^{(v-r)}$ & $b_{6}^{(v-r)}$ & $c_{6}^{(v-r)}$ & $a_{6}^{(v-r)}$ & $e_{6}^{(v-r)}$ & $f_{6}^{(v-r)}$ & $g_{6}^{(v-r)}$ \\
\hline 0 & 2 & 5 & 1 & 105 & 699 & 1398 & 699 & 179 & 179 & 279 \\
\hline 1 & 21 & 61 & 9 & 735 & 2097 & 4194 & 2097 & 959 & 959 & 837 \\
\hline 2 & 103 & 317 & 41 & 2625 & 3495 & 6990 & 3495 & 2519 & 2519 & 1395 \\
\hline 3 & 336 & 1061 & 129 & 6615 & 4893 & 9786 & 4893 & 4859 & 4859 & 1953 \\
\hline 4 & 852 & 2725 & 321 & 13545 & 6291 & 12582 & 6291 & 7979 & 7979 & 2511 \\
\hline 5 & 1827 & 5885 & 681 & 24255 & 7689 & 15378 & 7689 & 11879 & 11879 & 3069 \\
\hline 6 & 3481 & 11261 & 1289 & 39585 & 9087 & 18174 & 9087 & 16559 & 16559 & 3627 \\
\hline 7 & 6078 & 19717 & 2241 & 60375 & 10485 & 20970 & 10485 & 22019 & 22019 & 4185 \\
\hline 8 & 9926 & 32261 & 3649 & 87465 & 11883 & 23766 & 11883 & 28259 & 28259 & 4743 \\
\hline 9 & 15377 & 50045 & 5641 & 121695 & 13281 & 26562 & 13281 & 35279 & 35279 & 5301 \\
\hline 10 & 22827 & 74365 & 8361 & 163905 & 14679 & 29358 & 14679 & 43079 & 43079 & 5859 \\
\hline
\end{tabular}

TABLE E-3. Linear combination coefficients for $E^{(6)}$.

\begin{tabular}{|c|c|c|c|c|c|c|c|c|c|c|c|}
\hline$v$ & $\mathrm{~h}_{6}^{(\mathrm{v}-\mathrm{r})}$ & $\mathrm{i}_{6}^{(\mathrm{v}-\mathrm{r})}$ & $\mathrm{j}_{6}^{(\mathrm{v}-\mathrm{r})}$ & $\mathrm{k}_{6}^{(\mathrm{v}-\mathrm{r})}$ & $\mathrm{I}_{6}^{(\mathrm{v}-\mathrm{r})}$ & $\mathrm{m}_{6}^{(\mathrm{v}-\mathrm{r})}$ & $\mathrm{n}_{6}^{(\mathrm{v}-\mathrm{r})}$ & $\mathrm{O}_{6}^{(\mathrm{v}-\mathrm{r})}$ & $\mathrm{p}_{6}^{(\mathrm{v}-\mathrm{r})}$ & $\mathrm{q}_{6}^{(\mathrm{v}-\mathrm{r})}$ & $\mathrm{r}_{6}^{(\mathrm{v}-\mathrm{r})}$ \\
\hline 0 & 558 & 279 & 287 & 287 & 65 & 63 & 126 & 63 & 121 & 121 & 95 \\
\hline 1 & 1674 & 837 & 1787 & 1787 & 615 & 189 & 378 & 189 & 781 & 781 & 1035 \\
\hline 2 & 2790 & 1395 & 4787 & 4787 & 2425 & 315 & 630 & 315 & 2101 & 2101 & 4225 \\
\hline 3 & 3906 & 1953 & 9287 & 9287 & 6335 & 441 & 882 & 441 & 4081 & 4081 & 11165 \\
\hline 4 & 5022 & 2511 & 15287 & 15287 & 13185 & 567 & 1134 & 567 & 6721 & 6721 & 23355 \\
\hline 5 & 6138 & 3069 & 22787 & 22787 & 23815 & 693 & 1386 & 693 & 10021 & 10021 & 42295 \\
\hline 6 & 7254 & 3627 & 31787 & 31787 & 39065 & 819 & 1638 & 819 & 13981 & 13981 & 69485 \\
\hline 7 & 8370 & 4185 & 42287 & 42287 & 59775 & 945 & 1890 & 945 & 18601 & 18601 & 106425 \\
\hline 8 & 9486 & 4743 & 54287 & 54287 & 86785 & 1071 & 2142 & 1071 & 23881 & 23881 & 154615 \\
\hline 9 & 10602 & 5301 & 67787 & 67787 & 120935 & 1197 & 2394 & 1197 & 29821 & 29821 & 215555 \\
\hline 10 & 11718 & 5859 & 82787 & 82787 & 163065 & 1323 & 2646 & 1323 & 36421 & 36421 & 290745 \\
\hline
\end{tabular}


TABLEE-3. Linear combination coefficients for $E^{(6)}$.

\begin{tabular}{|c|c|c|c|c|c|c|c|c|c|c|c|c|}
\hline$v$ & $S_{6}^{(v-r)}$ & $t_{6}^{(v-r)}$ & $u_{6}^{(v-r)}$ & $\mathrm{V}_{6}^{(\mathrm{v}-\mathrm{r})}$ & $w_{6}^{(v-r)}$ & $x_{6}^{(v-r)}$ & $y_{6}^{(v-r)}$ & $z_{6}^{(v-r)}$ & $a a_{6}^{(v-r)}$ & $\mathrm{bb}_{6}^{(v-r)}$ & $\mathrm{CC}_{6}^{(\mathrm{v} \cdot \mathrm{r})}$ & $\mathrm{dd}_{6}^{(v-r)}$ \\
\hline 0 & 7 & 14 & 7 & 11 & 11 & 155 & 11 & 11 & 155 & 155 & 15 & 30 \\
\hline 1 & 21 & 42 & 21 & 71 & 71 & 1875 & 71 & 71 & 1875 & 1875 & 45 & 90 \\
\hline 2 & 35 & 70 & 35 & 191 & 191 & 7825 & 191 & 191 & 7825 & 7825 & 75 & 150 \\
\hline 3 & 49 & 98 & 49 & 371 & 371 & 20825 & 371 & 371 & 20825 & 20825 & 105 & 210 \\
\hline 4 & 63 & 126 & 63 & 611 & 611 & 43695 & 611 & 611 & 43695 & 43695 & 135 & 270 \\
\hline 5 & 77 & 154 & 77 & 911 & 911 & 79255 & 911 & 911 & 79255 & 79255 & 165 & 330 \\
\hline 6 & 91 & 182 & 91 & 1271 & 1271 & 130325 & 1271 & 1271 & 130325 & 130325 & 195 & 390 \\
\hline 7 & 105 & 210 & 105 & 1691 & 1691 & 199725 & 1691 & 1691 & 199725 & 199725 & 225 & 450 \\
\hline 8 & 119 & 238 & 119 & 2171 & 2171 & 290275 & 2171 & 2171 & 290275 & 290275 & 255 & 510 \\
\hline 9 & 133 & 266 & 133 & 2711 & 2711 & 404795 & 2711 & 2711 & 404795 & 404795 & 285 & 570 \\
\hline 10 & 147 & 294 & 147 & 3311 & 3311 & 546105 & 3311 & 3311 & 546105 & 546105 & 315 & 630 \\
\hline
\end{tabular}

TABLE E-4. Linear combination coefficients for $E^{(6)}$.

\begin{tabular}{|c|c|c|c|c|c|c|c|c|c|c|c|}
\hline$v$ & $e e_{6}^{(\mathrm{V}-\mathrm{r})}$ & $f f_{6}^{(v-r)}$ & $g g_{6}^{(v-r)}$ & $h h_{6}^{(v-r)}$ & $i i_{6}^{(v-r)}$ & $j j_{6}^{(v-r)}$ & $\mathrm{kk}_{6}^{(\mathrm{v}-\mathrm{I}}$ & $11_{6}^{(v-r)}$ & $\mathrm{mm}_{6}^{(\mathrm{v}-\mathrm{r})}$ & $\mathrm{nn}_{6}^{(\mathrm{v}-\mathrm{r})}$ & $0 O_{6}^{(v-r)}$ \\
\hline 0 & 15 & 151 & 151 & 35 & 11 & 22 & 11 & 31 & 31 & 19 & 53 \\
\hline 1 & 45 & 883 & 883 & 275 & 33 & 66 & 33 & 187 & 187 & 207 & 329 \\
\hline 2 & 75 & 2347 & 2347 & 1025 & 55 & 110 & 55 & 499 & 499 & 845 & 881 \\
\hline 3 & 105 & 4543 & 4543 & 2625 & 77 & 154 & 77 & 967 & 967 & 2233 & 1709 \\
\hline 4 & 135 & 7471 & 7471 & 5415 & 99 & 198 & 99 & 1591 & 1591 & 4671 & 2813 \\
\hline 5 & 165 & 11131 & 11131 & 9735 & 121 & 242 & 121 & 2371 & 2371 & 8459 & 4193 \\
\hline 6 & 195 & 15523 & 15523 & 15925 & 143 & 286 & 143 & 3307 & 3307 & 13897 & 5849 \\
\hline 7 & 225 & 20647 & 20647 & 24325 & 165 & 330 & 165 & 4399 & 4399 & 21285 & 7781 \\
\hline 8 & 255 & 26503 & 26503 & 35275 & 187 & 374 & 187 & 5647 & 5647 & 30923 & 9989 \\
\hline 9 & 285 & 33091 & 33091 & 49115 & 209 & 418 & 209 & 7051 & 7051 & 43111 & 12473 \\
\hline 10 & 315 & 40411 & 40411 & 66185 & 231 & 462 & 231 & 8611 & 8611 & 58149 & 15233 \\
\hline
\end{tabular}


TABLE E-5. Linear combination coefficients for $E^{(6)}$.

\begin{tabular}{|c|c|c|c|c|c|c|c|c|c|c|c|c|}
\hline$v$ & $\mathrm{PP}_{6}^{(\mathrm{v}-\mathrm{r})}$ & $q q_{6}^{(v-r)}$ & $\operatorname{rr}_{6}^{(v-r)}$ & $S S_{6}^{(v-r)}$ & $t t_{6}^{(v-r)}$ & $u_{u_{6}}^{(v-r)}$ & $V V_{6}^{(v-r)}$ & $w w_{6}^{(v-r)}$ & $\mathrm{XX}_{6}^{(v-r)}$ & $Y Y_{6}^{(v-r)}$ & $z z_{6}^{(v-r)}$ & $\operatorname{aaa}_{6}^{(v-r)}$ \\
\hline 0 & 53 & 133 & 1817 & 31 & 31 & 35 & 187 & 1 & 2 & 1 & 3 & 3 \\
\hline 1 & 329 & 1449 & 20541 & 187 & 187 & 275 & 1931 & 3 & 6 & 3 & 15 & 15 \\
\hline 2 & 881 & 5915 & 84535 & 499 & 499 & 1025 & 7785 & 5 & 10 & 5 & 39 & 39 \\
\hline 3 & 1709 & 15631 & 223979 & 967 & 967 & 2625 & 20489 & 7 & 14 & 7 & 75 & 75 \\
\hline 4 & 2813 & 32697 & 469053 & 1591 & 1591 & 5415 & 42783 & 9 & 18 & 9 & 123 & 123 \\
\hline 5 & 4193 & 59213 & 849937 & 2371 & 2371 & 9735 & 77407 & 11 & 22 & 11 & 183 & 183 \\
\hline 6 & 5849 & 97279 & 1396811 & 3307 & 3307 & 15925 & 127101 & 13 & 26 & 13 & 255 & 255 \\
\hline 7 & 7781 & 148995 & 2139855 & 4399 & 4399 & 24325 & 194605 & 15 & 30 & 15 & 339 & 339 \\
\hline 8 & 9989 & 216461 & 3109249 & 5647 & 5647 & 35275 & 282659 & 17 & 34 & 17 & 435 & 435 \\
\hline 9 & 12473 & 301777 & 4335173 & 7051 & 7051 & 49115 & 394003 & 19 & 38 & 19 & 543 & 543 \\
\hline 10 & 15233 & 407043 & 5847807 & 8611 & 8611 & 66185 & 531377 & 21 & 42 & 21 & 663 & 663 \\
\hline
\end{tabular}

TABLE E-6. Linear combination coefficients for $E^{(6)}$.

\begin{tabular}{|c|c|c|c|c|c|c|c|c|c|c|c|}
\hline$v$ & $\mathrm{bbb}_{6}^{(v-r)}$ & $\operatorname{CCC}_{6}^{(v-r)}$ & $\operatorname{ddd}_{6}^{(v-r)}$ & $\operatorname{eee}_{6}^{(v-r)}$ & $f f f_{6}^{(v-r)}$ & $\operatorname{ggg}_{6}^{(v-r)}$ & $\operatorname{hhh}_{6}^{(v-r)}$ & $i i i_{6}^{(v-r)}$ & $j j j_{6}^{(v-r)}$ & $k k_{k} k_{6}^{(v-r)}$ & $111_{6}^{(v-r)}$ \\
\hline 0 & 13 & 49 & 49 & 65 & 61 & 47 & 1 & 1 & 3 & 35 & 1 \\
\hline 1 & 123 & 277 & 277 & 615 & 618 & 411 & 5 & 5 & 21 & 309 & 7 \\
\hline 2 & 485 & 733 & 733 & 2425 & 2480 & 1585 & 13 & 13 & 75 & 1195 & 25 \\
\hline 3 & 1267 & 1417 & 1417 & 6335 & 6517 & 4109 & 25 & 25 & 189 & 3101 & 63 \\
\hline 4 & 2637 & 2329 & 2329 & 13185 & 13599 & 8523 & 41 & 41 & 387 & 6435 & 129 \\
\hline 5 & 4763 & 3469 & 3469 & 23815 & 24596 & 15367 & 61 & 61 & 693 & 11605 & 231 \\
\hline 6 & 7813 & 4837 & 4837 & 39065 & 40378 & 25181 & 85 & 85 & 1131 & 19019 & 377 \\
\hline 7 & 11955 & 6433 & 6433 & 59775 & 61815 & 38505 & 113 & 113 & 1725 & 29085 & 575 \\
\hline 8 & 17357 & 8257 & 8257 & 86785 & 89777 & 55879 & 145 & 145 & 2499 & 42211 & 833 \\
\hline 9 & 24187 & 10309 & 10309 & 120935 & 125134 & 77843 & 181 & 181 & 3477 & 58805 & 1159 \\
\hline 10 & 32613 & 12589 & 12589 & 163065 & 168756 & 104937 & 221 & 221 & 4683 & 79275 & 1561 \\
\hline
\end{tabular}


TABLEE-7. Symbolic term factors (87) for $E^{(6)}$.

\begin{tabular}{|c|c|c|c|c|c|c|c|c|c|c|c|c|c|}
\hline & $\ell_{1}^{\prime \prime}$ & $\ell_{2}^{\prime \prime}$ & $\ell_{3}^{\prime \prime}$ & $\ell_{4}^{\prime \prime}$ & $\ell_{5}^{\prime \prime}$ & $\ell_{6}^{\prime \prime}$ & $\ell_{7}^{\prime \prime}$ & $\ell_{8}^{\prime \prime}$ & $\ell_{9}^{\prime \prime}$ & $\ell_{10}^{\prime \prime}$ & $\ell_{11}^{\prime \prime}$ & $\ell_{12}^{\prime \prime}$ & $\Omega^{\prime \prime}$ \\
\hline$A_{6}^{(v)}$ & 0 & 0 & 0 & 6 & 0 & 0 & 0 & 0 & 0 & 9 & 0 & 5 & -5971968 \\
\hline$B_{6}^{(\mathrm{v})}$ & 0 & 0 & 0 & 4 & 1 & 0 & 0 & 0 & 0 & 8 & 0 & 4 & 663552 \\
\hline$C_{6}^{(\mathrm{v})}$ & 0 & 0 & 0 & 2 & 2 & 0 & 0 & 0 & 0 & 7 & 0 & 3 & -663552 \\
\hline$D_{6}^{(\mathrm{v})}$ & 0 & 0 & 0 & 0 & 3 & 0 & 0 & 0 & 0 & 6 & 0 & 2 & 24576 \\
\hline$E_{6}^{(\mathrm{v})}$ & 0 & 0 & 0 & 3 & 0 & 1 & 0 & 0 & 0 & 7 & 0 & 3 & -414720 \\
\hline$F_{6}^{(v)}$ & 0 & 0 & 0 & 1 & 1 & 1 & 0 & 0 & 0 & 6 & 0 & 2 & 23040 \\
\hline$G_{6}^{(\mathrm{v})}$ & 0 & 0 & 0 & 0 & 0 & 2 & 0 & 0 & 0 & 5 & 0 & 1 & -460800 \\
\hline$H_{6}^{(\mathrm{v})}$ & 0 & 0 & 0 & 2 & 0 & 0 & 1 & 0 & 0 & 6 & 0 & 2 & 82944 \\
\hline$I_{6}^{(\mathrm{v})}$ & 0 & 0 & 0 & 0 & 1 & 0 & 1 & 0 & 0 & 5 & 0 & 1 & -1536 \\
\hline$J_{6}^{(\mathrm{v})}$ & 0 & 0 & 0 & 1 & 0 & 0 & 0 & 1 & 0 & 5 & 0 & 1 & -4608 \\
\hline$K_{6}^{(v)}$ & 0 & 0 & 0 & 0 & 0 & 0 & 0 & 0 & 1 & 4 & 0 & 0 & 6144 \\
\hline$A_{6}^{(\mathrm{v}-\mathrm{r})}$ & 1 & 1 & 1 & 0 & 0 & 0 & 0 & 0 & 0 & 3 & 6 & 0 & 8 \\
\hline$B_{6}^{(\mathrm{v}-\mathrm{r})}$ & 3 & 1 & 3 & 0 & 0 & 0 & 0 & 0 & 0 & 3 & 6 & 2 & 4 \\
\hline$C_{6}^{(v-\mathrm{r})}$ & 4 & 1 & 3 & 0 & 0 & 0 & 0 & 0 & 0 & 3 & 6 & 2 & 4 \\
\hline$D_{6}^{(\mathrm{v}-\tau)}$ & 5 & 1 & 3 & 0 & 0 & 0 & 0 & 0 & 0 & 3 & 6 & 2 & 4 \\
\hline$E_{6}^{(\mathrm{V}-\mathrm{r})}$ & 2 & 1 & 2 & 0 & 0 & 0 & 0 & 0 & 0 & 3 & 6 & 1 & -2 \\
\hline$F_{6}^{(v-r)}$ & 3 & 1 & 2 & 0 & 0 & 0 & 0 & 0 & 0 & 3 & 6 & 1 & -2 \\
\hline$G_{6}^{(\mathrm{v}-\mathrm{r})}$ & 3 & 1 & 3 & 1 & 0 & 0 & 0 & 0 & 0 & 4 & 5 & 3 & 4 \\
\hline$H_{6}^{(\mathrm{v}-\mathrm{r})}$ & 4 & 1 & 3 & 1 & 0 & 0 & 0 & 0 & 0 & 4 & 5 & 3 & 4 \\
\hline$I_{6}^{(\mathrm{v}-\mathrm{r})}$ & 5 & 1 & 3 & 1 & 0 & 0 & 0 & 0 & 0 & 4 & 5 & 3 & 4 \\
\hline$J_{6}^{(\mathrm{v}-\tau)}$ & 2 & 1 & 2 & 1 & 0 & 0 & 0 & 0 & 0 & 4 & 5 & 2 & -6 \\
\hline$K_{6}^{(v-r)}$ & 3 & 1 & 2 & 1 & 0 & 0 & 0 & 0 & 0 & 4 & 5 & 2 & -6 \\
\hline$L_{6}^{(\mathrm{v}-\mathrm{r})}$ & 1 & 1 & 1 & 1 & 0 & 0 & 0 & 0 & 0 & 4 & 5 & 1 & 8 \\
\hline$M_{6}^{(\mathrm{v}-\mathrm{r})}$ & 3 & 1 & 3 & 2 & 0 & 0 & 0 & 0 & 0 & 5 & 4 & 4 & 4 \\
\hline$N_{6}^{(v-\mathrm{r})}$ & 4 & 1 & 3 & 2 & 0 & 0 & 0 & 0 & 0 & 5 & 4 & 4 & 4 \\
\hline$O_{6}^{(\mathrm{v}-\mathrm{r})}$ & 5 & 1 & 3 & 2 & 0 & 0 & 0 & 0 & 0 & 5 & 4 & 4 & 4 \\
\hline
\end{tabular}


TABLE E-8. Symbolic term factors (87) for $E^{(6)}$.

\begin{tabular}{|c|c|c|c|c|c|c|c|c|c|c|c|c|c|}
\hline & $\ell_{1}^{\prime \prime}$ & $\ell_{2}^{\prime \prime}$ & $\ell_{3}^{\prime \prime}$ & $\ell_{4}^{\prime \prime}$ & $\ell_{5}^{\prime \prime}$ & $\ell_{6}^{\prime \prime}$ & $\ell_{7}^{\prime \prime}$ & $\ell_{8}^{\prime \prime}$ & $\ell_{9}^{\prime \prime}$ & $\ell_{10}^{\prime \prime}$ & $\ell_{11}^{\prime \prime}$ & $\ell_{12}^{\prime \prime}$ & $\Omega^{\prime \prime}$ \\
\hline$P_{6}^{(\mathrm{v}-\mathrm{r})}$ & 2 & 1 & 2 & 2 & 0 & 0 & 0 & 0 & 0 & 5 & 4 & 3 & -8 \\
\hline$Q_{6}^{(v-r)}$ & 3 & 1 & 2 & 2 & 0 & 0 & 0 & 0 & 0 & 5 & 4 & 3 & -8 \\
\hline$R_{6}^{(\mathrm{v}-\mathrm{I})}$ & 1 & 1 & 1 & 2 & 0 & 0 & 0 & 0 & 0 & 5 & 4 & 2 & 32 \\
\hline$S_{6}^{(v-r)}$ & 3 & 1 & 3 & 3 & 0 & 0 & 0 & 0 & 0 & 6 & 3 & 5 & 4 \\
\hline$T_{6}^{(\mathrm{v}-\mathrm{r})}$ & 4 & 1 & 3 & 3 & 0 & 0 & 0 & 0 & 0 & 6 & 3 & 5 & 4 \\
\hline$U_{6}^{(\mathrm{v}-\mathrm{r})}$ & 5 & 1 & 3 & 3 & 0 & 0 & 0 & 0 & 0 & 6 & 3 & 5 & 4 \\
\hline$V_{6}^{(\mathrm{V}-\mathrm{I})}$ & 2 & 1 & 2 & 3 & 0 & 0 & 0 & 0 & 0 & 6 & 3 & 4 & -3 \\
\hline$W_{6}^{(v-r)}$ & 3 & 1 & 2 & 3 & 0 & 0 & 0 & 0 & 0 & 6 & 3 & 4 & -3 \\
\hline$X_{6}^{(\mathrm{v}-\mathrm{r})}$ & 1 & 1 & 1 & 3 & 0 & 0 & 0 & 0 & 0 & 6 & 3 & 3 & 144 \\
\hline$Y_{6}^{(\mathbf{v - r})}$ & 2 & 1 & 2 & 4 & $\overline{0}$ & 0 & 0 & 0 & 0 & 7 & 2 & 5 & -18 \\
\hline$Z_{6}^{(v-r)}$ & 3 & 1 & 2 & 4 & 0 & 0 & 0 & 0 & 0 & 7 & 2 & 5 & -18 \\
\hline$A A_{6}^{(\mathrm{v}-\mathrm{r})}$ & 1 & 1 & 1 & 4. & 0 & 0 & 0 & 0 & 0 & 7 & 2 & 4 & 512 \\
\hline$B B_{6}^{(\mathrm{v}-\mathrm{r})}$ & 1 & 1 & 1 & 5 & 0 & 0 & 0 & 0 & 0 & 8 & 1 & 5 & 1536 \\
\hline$C C_{6}^{(\mathrm{v}-\mathrm{r})}$ & 3 & 1 & 3 & 0 & 1 & 0 & 0 & 0 & 0 & 4 & 4 & 3 & -2 \\
\hline$D D_{6}^{(\mathrm{v}-\mathrm{r})}$ & 4 & 1 & 3 & 0 & 1 & 0 & 0 & 0 & 0 & 4 & 4 & 3 & -2 \\
\hline$E E_{6}^{(\mathrm{v}-r)}$ & 5 & 1 & 3 & 0 & 1 & 0 & 0 & 0 & 0 & 4 & 4 & 3 & -2 \\
\hline$F F_{6}^{(\mathrm{v}-\mathrm{r})}$ & 2 & 1 & 2 & 0 & 1 & 0 & 0 & 0 & 0 & 4 & 4 & 2 & 24 \\
\hline$G G_{6}^{(\mathrm{v}-\mathrm{s})}$ & 3 & 1 & 2 & 0 & 1 & 0 & 0 & 0 & 0 & 4 & 4 & 2 & 24 \\
\hline$H H_{6}^{(\mathrm{v}-\mathrm{r})}$ & 1 & 1 & 1 & 0 & 1 & 0 & 0 & 0 & 0 & 4 & 4 & 1 & -32 \\
\hline$I_{6}^{(\mathrm{v}-\mathrm{r})}$ & 3 & 1 & 3 & 1 & 1 & 0 & 0 & 0 & 0 & 5 & 3 & 4 & -6 \\
\hline$J J_{6}^{(v-r)}$ & 4 & 1 & 3 & 1 & 1 & 0 & 0 & 0 & 0 & 5 & 3 & 4 & -6 \\
\hline$K K_{6}^{(\mathrm{y}-\mathrm{r})}$ & 5 & 1 & 3 & 1 & 1 & 0 & 0 & 0 & 0 & 5 & 3 & 4 & -6 \\
\hline$L L_{6}^{(\mathrm{v}-\mathrm{r})}$ & 2 & 1 & 2 & 1 & 1 & 0 & 0 & 0 & 0 & 5 & 3 & 3 & 8 \\
\hline$M M_{6}^{(\mathrm{v}-\mathrm{r})}$ & 3 & 1 & 2 & 1 & 1 & 0 & 0 & 0 & 0 & 5 & 3 & 3 & 8 \\
\hline$N N_{6}^{(\mathrm{v}-\mathrm{I})}$ & 1 & 1 & 1 & 1 & 1 & 0 & 0 & 0 & 0 & 5 & 3 & 2 & -16 \\
\hline$O O_{6}^{(\mathrm{v}-\mathrm{r})}$ & 2 & 1 & 2 & 2 & 1 & 0 & 0 & 0 & 0 & 6 & 2 & 4 & 48 \\
\hline$P P_{6}^{(\mathrm{v}-\mathrm{r})}$ & 3 & 1 & 2 & 2 & 1 & 0 & 0 & 0 & 0 & 6 & 2 & 4 & 48 \\
\hline
\end{tabular}


TABLE E-9. Symbolic term factors (87) for $E^{(6)}$.

\begin{tabular}{|c|c|c|c|c|c|c|c|c|c|c|c|c|c|}
\hline & $\overline{\ell_{1}^{\prime \prime}}$ & $\ell_{2}^{\prime \prime}$ & $\ell_{3}^{\prime \prime}$ & $\ell_{4}^{\prime \prime}$ & $\ell_{5}^{\prime \prime}$ & $\ell_{6}^{\prime \prime}$ & $\ell_{7}^{\prime \prime}$ & $\ell_{8}^{\prime \prime}$ & $\ell_{9}^{\prime \prime}$ & $\ell_{10}^{\prime \prime}$ & $\ell_{11}^{\prime \prime}$ & $\ell_{12}^{\prime \prime}$ & $\Omega^{\prime \prime}$ \\
\hline$Q Q_{6}^{(\mathrm{v}-\mathrm{r})}$ & 1 & 1 & 1 & 2 & 1 & 0 & 0 & 0 & 0 & 6 & 2 & 3 & -256 \\
\hline$R R_{6}^{(\mathrm{v}-\tau)}$ & 1 & 1 & 1 & 3 & 1 & 0 & 0 & 0 & 0 & 7 & 1 & 4 & -6912 \\
\hline$S S_{6}^{(\mathrm{v}-\mathrm{r})}$ & 2 & 1 & 2 & 0 & 2 & 0 & 0 & 0 & 0 & 5 & 2 & 3 & -144 \\
\hline$T T_{6}^{(\mathrm{v}-\mathrm{r})}$ & 3 & 1 & 2 & 0 & 2 & 0 & 0 & 0 & 0 & 5 & 2 & 3 & -144 \\
\hline$U U_{6}^{(\mathrm{v}-\mathrm{r})}$ & 1 & 1 & 1 & 0 & 2 & 0 & 0 & 0 & 0 & 5 & 2 & 2 & 512 \\
\hline$V V_{6}^{(\mathrm{v}-\mathrm{r})}$ & 1 & 1 & 1 & 1 & 2 & 0 & 0 & 0 & 0 & 6 & 1 & 3 & 1536 \\
\hline$W W_{0}^{(\mathrm{v}-\mathrm{r})}$ & 3 & 1 & 3 & 0 & 0 & 1 & 0 & 0 & 0 & 4 & 3 & 3 & 3 \\
\hline$X X_{6}^{(v \cdot r)}$ & 4 & 1 & 3 & 0 & 0 & 1 & 0 & 0 & 0 & 4 & 3 & 3 & 3 \\
\hline$Y Y_{6}^{(\mathrm{v}-\mathrm{r})}$ & 5 & 1 & 3 & 0 & 0 & 1 & 0 & 0 & 0 & 4 & 3 & 3 & 3 \\
\hline$Z Z_{6}^{(v-r)}$ & 2 & 1 & 2 & 0 & 0 & 1 & 0 & 0 & 0 & 4 & 3 & 2 & -4 \\
\hline$A A A_{6}^{(\mathrm{v}-r)}$ & 3 & 1 & 2 & 0 & 0 & 1 & 0 & 0 & 0 & 4 & 3 & 2 & -4 \\
\hline$B B B_{6}^{(v-r)}$ & 1 & 1 & 1 & 0 & 0 & 1 & 0 & 0 & 0 & 4 & 3 & 1 & 48 \\
\hline$C C C_{6}^{(\mathrm{v} \cdot r)}$ & 2 & 1 & 2 & 1 & 0 & 1 & 0 & 0 & 0 & 5 & 2 & 3 & -144 \\
\hline$D D D_{6}^{(\mathrm{v}-\mathrm{r})}$ & 3 & 1 & 2 & 1 & 0 & 1 & 0 & 0 & 0 & 5 & 2 & 3 & -144 \\
\hline$E E E_{6}^{(v-r)}$ & 1 & 1 & 1 & 1 & 0 & 1 & 0 & 0 & 0 & 5 & 2 & 2 & 384 \\
\hline$F F F_{6}^{(\mathrm{v}-\mathrm{r})}$ & 1 & 1 & 1 & 2 & 0 & 1 & 0 & 0 & 0 & 6 & 1 & 3 & 576 \\
\hline$G G G_{6}^{(v-1)}$ & 1 & 1 & 1 & 0 & 1 & 1 & 0 & 0 & 0 & 5 & 1 & 2 & -1152 \\
\hline$H H H_{6}^{(\mathrm{v}-\mathrm{r})}$ & 2 & 1 & 2 & 0 & 0 & $\overline{0}$ & 1 & 0 & 0 & 4 & 2 & 2 & 16 \\
\hline$I I_{6}^{(\mathrm{v}-\mathrm{r})}$ & 3 & 1 & 2 & 0 & 0 & 0 & 1 & 0 & 0 & 4 & 2 & 2 & 16 \\
\hline$J J J_{6}^{(\mathrm{v}-r)}$ & 1 & 1 & 1 & 0 & 0 & 0 & 1 & 0 & 0 & 4 & 2 & 1 & -128 \\
\hline$K K K_{6}^{(v-r)}$ & 1 & 1 & 1 & 1 & 0 & 0 & 1 & 0 & 0 & 5 & 1 & 2 & -1152 \\
\hline$L L L_{6}^{(\mathrm{v}-\mathrm{r})}$ & 1 & 1 & 1 & 0 & 0 & 0 & 0 & 1 & 0 & 4 & 1 & 1 & 192 \\
\hline
\end{tabular}


Hulburt-Hirschfelder Force Constants

$$
\begin{aligned}
& k_{e}=2 D \beta^{2} \\
& k_{3}=6 D \beta^{3}(c-1) \\
& k_{4}=2 D \beta^{4}(12 b c-24 c+7) \\
& k_{5}=30 D \beta^{5}(-8 b c+8 c-1) \\
& k_{6}=2 D \beta^{6}(720 b c-480 c+31) \\
& k_{7}=42 D \beta^{7}(-160 b c+80 c-3) \\
& k_{8}=2 D \beta^{8}(13440 b c-5376 c+127) \\
& k_{9}=6 D \beta^{9}(-16128 b c+5376 c-85) \\
& k_{10}=2 D \beta^{10}(161280 b c-46080 c+511)
\end{aligned}
$$




\section{REFERENCES}

[1] Wilson, E. B., Jr.; Decius, J. C.; and P. C. Cross. Molecular Vibrations. New York: Dover, 1980.

[2] Ermler, W. C.; and H. C. Hsieh. "Analytical Representation and Vibrational-Rotational Analysis of $a b$ Initio Potential Energy and Property Surfaces," in Advances in Molecular Electronic Structure Theory, Volume I: Calculation and Characterization of Molecular Potential Energy Surfaces. Edited by T. H. Dunning, Jr. Greenwich: JAI Press, 1990.

[3] Levine, I. N. Quantum Chemistry, 2nd ed. Boston: Allyn and Bacon, 1974.

[4] Harding, L. B.; and W. C. Ermler. "Polyatomic, Anharmonic, Vibrational-Rotational Analysis. Application to Accurate ab Initio Results for Formaldehyde." Journal of Computational Chemistry, 1985, 6, 13.

[5] Hargiss, L. O.; and W. C. Ermler. "Vibrational-Rotational Analysis of ab Initio Potential Energy Surfaces for Symmetric-Top Molecules: Application to Ammonia Isotopomers." Journal of Physical Chemistry, 1988, 92, 300.

[6] Pilar, F. L. Elementary Quantum Chemistry. New York: McGraw-Hill, 1968.

[7] Hirschfelder, J. O.; Byers Brown, W.; and S. T. Epstein. "Recent Developments in Perturbation Theory," in Advances in Quantum Chemistry, V. 1. Edited by P. Löwdin. New York: Academic Press, 1964.

[8] Sprandel, L. L.; and C. W. Kern. "A Test of Perturbation Theory for Determining Anharmonic Vibrational Corrections to Properties of Diatomic Molecules." Molecular Physics, 1972, 24, 1383.

[9] Dudas, M. M.; Hsieh, H. C.; and W. C. Ermler. "Application of Quantum Mechanical Perturbation Theory to Molecular Vibrational-Rotational Analysis." Mathematica Journal, $1992,2,66$.

[10] Krohn, B. J.; Ermler, W. C.; and C. W. Kern. "Nuclear Corrections to Molecular Properties. IV. Theory for Low-Lying Vibrational States of Polyatomic Molecules with Application to the Water Molecule Near the Hartree-Fock Limit." Journal of Chemical Physics, 1974, 60, 22.

[11] Murrell, J. N.; Carter, S.; Farantos, S. C.; Huxley, P.; and A. J. C. Varandas. Molecular Potential Energy Functions. Chichester: John Wiley \& Sons, 1984.

[12] Mulliken, R. S.; and W. C. Ermler. Diatomic Molecules: Results of ab Initio Calculations. New York: Academic Press, 1977. 
[13] Mulliken, R. S.; and W. C. Ermler. Polyatomic Molecules: Results of ab Initio Calculations. New York: Academic Press, 1981.

[14] Dunning, T. H., Jr., ed. Advances in Molecular Electronic Structure Theory, Volume I: Calculation and Characterization of Molecular Potential Energy Surfaces. Greenwich, JAI Press, 1990.

[15] Born, M.; and J. R. Oppenheimer. "Zur Quantentheorie der Molekeln." Annalen der Physik, 1927, 84, 457.

[16] Ermler, W. C.; Hsieh, H. C.; and L. B. Harding. "Polyatomic Surface Fitting, VibrationalRotational Analysis, Expectation Value and Intensity Program.” Computer Physics Communications, 1988, 51, 257.

[17] Levine, I. N. Molecular Spectroscopy. New York: John Wiley \& Sons, 1975.

[18] Sanders, F. C.; and C. W. Scherr. "Perturbation Study of Some Excited States of TwoElectron Atoms." Physical Review, 1969, 181, 84.

[19] Adams, B. G.; and G. A. Arteca. "Symbolic Computation of Energy Perturbation Expansions for Spherically Symmetric Hydrogenic Systems." Computers in Physics, 1994, 8,343 .

[20] Adams, B. G. "Unified Treatment of High-Order Perturbation Theory for the Stark Effect in a Two- and Three-Dimensional Hydrogen Atom." Physical Review Series A, 1992, 46, 4060 .

[21] Fernández, F. M. "Large-Order Perturbation Theory without a Wavefunction for the LoSurdo-Stark Effect in Hydrogenic Atoms." Journal of Physics Series A, 1992, 25, 495.

[22] McRae, S. M.; and E. R. Vrscay. "Canonical Perturbation Expansions to Large Order from Classical Hypervirial and Hellmann-Feynman Theorems." Journal of Mathematical Physics, 1992, 33, 3004.

[23] Vinette, F.; and J. Č́ǐ̌ek. "Perturbation Energy Expansion Using Hypervirial Theorem and Symbolic Computation for the $N$-Dimensional Hydrogen Atom in an External Spherically Symmetric Field." Computer Physics Communications, 1988, 52, 35.

[24] Ogilvie, J. F. "Computer Algebra in Modern Physics.” Computers in Physics, 1989, 3, 66.

[25] Bouanich, J. P. “Analytic Vibration-Rotational Matrix Elements for High $\Delta v$ Infrared Transitions of Diatomic Molecules." Journal of Quantitative Spectroscopy and Radiative Transfer, 1987, 38, 89.

[26] Herbert, J. M. “A General Formula for Rayleigh-Schrödinger Perturbation Energy Utilizing 
a Power Series Expansion of the Quantum Mechanical Hamiltonian." Technical Memorandum No. 222, Argonne National Laboratory, Division of Mathematics and Computer Science, 1997.

[27] Dennison, D. M.; and K. T. Hecht. "Molecular Spectra," in Quantum Theory, Volume II: Aggregates of Particles. Edited by D. R. Bates. New York: Academic Press, 1962.

[28] Townes, C. H.; and A. L. Schawlow. Microwave Spectroscopy. New York: McGraw-Hill, 1955.

[29] Barrow, G. M. Introduction to Molecular Spectroscopy. New York: McGraw-Hill, 1962.

[30] Gillette, P. Calculus and Analytic Geometry, 3rd ed. Lexington: D. C. Heath and Company, 1988.

[31] Ogilvie, J. F. “A General Potential Energy Function for Diatomic Molecules." Proceedings of the Royal Society of London Series A, 1981, 378, 287; Errata, 1982, 381, 479.

[32] Huber, K. P.; and G. Herzberg. Molecular Spectra and Molecular Structure, Volume IV: Constants of Diatomic Molecules. New York: Van Nostrand Reinhold, 1979.

[33] Kolos, W.; and L. Wolniewicz. "Accurate Adiabatic Treatment of the Ground State of the Hydrogen Molecule." Journal of Chemical Physics, 1964, 41, 3663.

[34] King, G. W. Spectroscopy and Molecular Structure. New York: Holt, Rinehart, and Winston, 1964.

[35] Nielsen, H. H. "The Vibration-Rotation Energies of Molecules." Reviews of Modern Physics, 1951, 23, 90.

[36] Goldsmith, M.; Amat, G.; and H. H. Nielsen. "Higher Order Rotation-Vibration Energies of Polyatomic Molecules I." Journal of Chemical Physics, 1956, 24, 1178.

[37] Amat, G.; Nielsen, H. H.; and G. Tarrago. Rotation-Vibration of Polyatomic Molecules. New York: Marcel Dekker, 1971.

[38] Kern, C. W.; and R. L. Matcha. "Nuclear Corrections to Electronic Expectation Values: Zero-Point Vibrational Effects in the Water Molecule." Journal of Chemical Physics, 1968, 49, 2081.

[39] Matsen, F. A. Vector Spaces and Algebras for Chemistry and Physics. New York: Holt, Rinehart, and Winston, 1970.

[40] Dalgarno, A. "Stationary Perturbation Theory," in Quantum Theory, Volume I: Elements. Edited by D. R. Bates. New York: Academic Press, 1961. 
[41] Dunham, J. L. "The Energy Levels of a Rotating Vibrator." Physical Review, 1932, 41, 721.

[42] Darling, B. T.; and D. M. Dennison. "The Water Vapor Molecule." Physical Review, $1939,57,128$.

[43] Ogilvie, J. F. "Spectroscopic Energy Coefficients for Vibration-Rotational States of Dinuclear Molecules." Computer Physics Communications, 1983, 30, 101.

[44] Jones, H. W. "Developments in Multicenter Molecular Integrals Over STOs Using Expansions in Spherical Harmonics." International Joumal of Quantum Chemistry, 1994, 51,417 .

[45] Steele, D.; Lippincott, E. R.; and J. T. Vanderslice. "Comparative Study of Empirical Internuclear Potential Functions." Reviews of Modern Physics, 1962, 34, 239.

[46] Morse, P. M. "Diatomic Molecules According to the Wave Mechanics. II. Vibrational Levels." Physical Review, 1929, 34, 57.

[47] Hulburt, H. M.; and J. O. Hirschfelder. "Potential Energy Functions for Diatomic Molecules." Journal of Chemical Physics, 1941, 9, 61; Errata, 1961, 35, 1901.

[48] Herzberg, G. Molecular Spectra and Molecular Structure, Volume I: Spectra of Diatomic Molecules, 2nd ed. Toronto: Van Nostrand, 1950.

[49] Bunker, P. R. "On the Breakdown of the Born-Oppenheimer Approximation for a Diatomic Molecule." Journal of Molecular Spectroscopy, 1972, 5, 478. 
Distribution for ANL-97/11

Internal:
J. M. Beumer (10)
F. Y. Fradin
J. M. Herbert (25)
G. W. Pieper
R. L. Stevens
C. L. Wilkinson
TIS File

\section{External:}

DOE-0STI, for distribution per UC-405 (52)

ANL-E Library

ANL-W Library

Manager, Chicago Operations Office, DOE

D. Hitchcock, DOE - Mathematical, Information, and Computational Sciences Div.

F. Howes, DOE - Mathematical, Information, and Computational Sciences Div.

D. Nelson, DOE - Office of Energy Research 